\title{
Systematic review with meta-analysis of the epidemiological evidence relating smoking to COPD, chronic bronchitis and emphysema
}

\author{
Barbara A Forey ${ }^{1 *}$, Alison J Thornton ${ }^{2}$ and Peter N Lee ${ }^{1}$
}

\begin{abstract}
Background: Smoking is a known cause of the outcomes COPD, chronic bronchitis (CB) and emphysema, but no previous systematic review exists. We summarize evidence for various smoking indices.

Methods: Based on MEDLINE searches and other sources we obtained papers published to 2006 describing epidemiological studies relating incidence or prevalence of these outcomes to smoking. Studies in children or adolescents, or in populations at high respiratory disease risk or with co-existing diseases were excluded. Studyspecific data were extracted on design, exposures and outcomes considered, and confounder adjustment. For each outcome RRs/ORs and 95\% Cls were extracted for ever, current and ex smoking and various dose response indices, and meta-analyses and meta-regressions conducted to determine how relationships were modified by various study and RR characteristics.
\end{abstract}

Results: Of 218 studies identified, 133 provide data for COPD, 101 for CB and 28 for emphysema. RR estimates are markedly heterogeneous. Based on random-effects meta-analyses of most-adjusted RR/ORs, estimates are elevated for ever smoking (COPD 2.89, Cl 2.63-3.17, $\mathrm{n}=129$ RRs; CB 2.69, 2.50-2.90, $\mathrm{n}=114$; emphysema 4.51, 3.38-6.02, $\mathrm{n}=$ 28), current smoking (COPD 3.51, 3.08-3.99; CB 3.41, 3.13-3.72; emphysema 4.87, 2.83-8.41) and ex smoking (COPD 2.35, 2.11-2.63; CB 1.63, 1.50-1.78; emphysema 3.52, 2.51-4.94). For COPD, RRs are higher for males, for studies conducted in North America, for cigarette smoking rather than any product smoking, and where the unexposed base is never smoking any product, and are markedly lower when asthma is included in the COPD definition. Variations by sex, continent, smoking product and unexposed group are in the same direction for CB, but less clearly demonstrated. For all outcomes RRs are higher when based on mortality, and for COPD are markedly lower when based on lung function. For all outcomes, risk increases with amount smoked and pack-years. Limited data show risk decreases with increasing starting age for COPD and CB and with increasing quitting duration for COPD. No clear relationship is seen with duration of smoking.

Conclusions: The results confirm and quantify the causal relationships with smoking.

\section{Background}

It has been known for many years that smoking causes chronic obstructive pulmonary disease (COPD). In 1984, the US Surgeon General [1] concluded that, in the United States, 80 to $90 \%$ of morbidity from COPD is attributable to cigarette smoking. However, we know of no previous systematic review quantifying this relationship by metaanalysis, and we attempt to rectify this omission. It is

\footnotetext{
* Correspondence: BarbaraForey@pnlee.co.uk

${ }^{1} \mathrm{P}$ N Lee Statistics and Computing Ltd, Sutton, Surrey, UK

Full list of author information is available at the end of the article
}

recognized [1] that COPD comprises three separate, often interconnected disease processes: (1) airway thickening and narrowing with expiratory airflow obstruction; (2) chronic mucus hypersecretion, resulting in chronic cough and phlegm production; and (3) emphysema, an abnormal dilation of distal airspaces combined with destruction of alveolar walls. The present review considers all three processes by summarizing the epidemiological evidence relating smoking separately to the incidence or prevalence of COPD, chronic bronchitis $(\mathrm{CB})$ and emphysema. Elsewhere [2], we systematically review

\section{Biomed Central}

(c) 2011 Forey et al; licensee BioMed Central Ltd. This is an Open Access article distributed under the terms of the Creative Commons Attribution License (http://creativecommons.org/licenses/by/2.0), which permits unrestricted use, distribution, and reproduction in any medium, provided the original work is properly cited. 
evidence on the relationship between smoking and decline in forced expiratory volume in one second $\left(\mathrm{FEV}_{1}\right)$.

Because COPD is rarely seen in children or adolescents, we restrict attention to adults. We also limit attention to studies of the general population, so do not, for example, consider studies in subjects suffering from alpha-1 antitrypsin deficiency or exposed to particular respiratory hazards. To provide a broad description of the relationship, we do not concentrate on one primary analysis, but quantify the relationship of each of the three outcomes studied (COPD, CB, emphysema) to each of a range of indices of smoking, investigating how these relationships vary according to characteristics such as sex, age, location, study design, period considered, definition of outcome, definition of exposure and extent of confounder adjustment.

\section{Methods}

Full details of the methods used are described in Additional file 1 , and are summarized below.

\section{Inclusion and exclusion criteria}

Attention was restricted to epidemiological studies published before 2007 on COPD, CB or emphysema, providing relative risk (RR) estimates for one or more defined "major indices" (ever, current or ex smoking compared with never smoking) or "dose-related indices" (amount smoked, age of starting to smoke, pack-years smoked, duration of smoking or duration of quitting). Throughout this paper, we use the term RR to include its various estimators, including the odds ratio and the hazard ratio.

Studies were excluded if in children or adolescents, or in subjects at especially high risk of respiratory disease (e.g. workers in risky occupations), selected as having co-existing diseases or conditions, or from atypical populations likely to have a highly unusual prevalence of smoking or disease. Also excluded were uncontrolled case studies, and studies of disease exacerbation or undiagnosed disease, of symptom-free subjects, or where the only results were adjusted for symptoms or precursors of disease.

\section{Definition of the outcomes COPD}

The term COPD is quite recent, so studies with outcomes described otherwise were also included. These could be based on International Classification of Diseases (ICD) codes, on lung function criteria, on a combination of lung function criteria and symptoms, or on combinations of diagnosed conditions (such as CB or emphysema, or $\mathrm{CB}$, emphysema or asthma), where diagnoses were extracted from medical records or reported in questionnaires. Unacceptable outcomes included $\mathrm{CB}$ or emphysema separately, acute or unspecified bronchitis, non-specific respiratory disease, or outcomes based only on symptoms and not on lung function. The range of ICD codes had to cover both CB and emphysema, and could also cover asthma, acute and unqualified bronchitis, bronchiectasis and some other defined lung conditions. Broader-ranging definitions (e.g. respiratory disease) were not accepted. Acceptable lung function criteria included those of the Global Initiative for Chronic Obstructive Lung Disease (GOLD) [3,4], the British Thoracic Society (BTS) [5], the European Respiratory Society (ERS) [6] and the American Thoracic Society (ATS) [7-9]. Use of a bronchodilator was not a requirement.

$C B$

Where based on the ICD, the range had to include the code(s) for CB and could also include codes for acute or unspecified bronchitis. Acceptable outcomes could also be based on medical records, in-study diagnosis, selfreport of physician diagnosis or of history of the disease, or on symptoms. The British Medical Research Council (MRC) criterion of daily productive cough for at least three consecutive months for more than two successive years $[10,11]$ was recognized as a set of symptoms defining CB. Diagnoses or symptoms called "bronchitis" were accepted where the context clearly indicated it was chronic. Diagnoses based on symptoms not referred to as $\mathrm{CB}$ were also accepted, provided the definition included both chronic cough and phlegm.

\section{Emphysema}

The outcome could be based on the ICD code for emphysema, on medical records, in-study diagnosis, or on self-report of physician diagnosis or history of the disease.

\section{Choice of outcome}

Where a study provided data for multiple acceptable definitions of an outcome, results were entered only for one. Additional file 1 gives the rules specifying choice of outcome, and, for studies providing a choice, lists definitions selected and rejected. It also gives, for all studies, the description of the disease and the source of the diagnosis for all outcomes where data were entered.

\section{Literature searching}

Searching was carried out in phases. Initially, 1407 potentially relevant papers, published up to 2002, were derived by AJT from an unpublished project which used the $\mathrm{MeSH}$ terms chronic bronchitis and symptoms, emphysema, lung function, genetic determinants, mortality, adults and smoking. Subsequently, additional Medline searches were conducted in 2006 by AJT and in 2008 by BAF, using the MeSH term "Pulmonary disease, chronic obstructive". Papers were also sought from 
in-house files on smoking and health, and references cited in papers obtained. Publications before 2007 were considered, with no restriction on language or on peerreviewed journals. Reasons for rejection were recorded.

\section{Identification of studies}

Relevant papers were allocated to studies, noting multiple papers on the same study, and papers reporting on multiple studies. Each study was given a unique reference code (REF) of up to 6 characters (e.g. DICKIN or CHEN3), based on the principal author's name, and distinguishing multiple studies by the same author. Occasionally, an original study was split into separate studies (e.g. where follow-up periods differed by sex).

Some studies were noted as having overlaps or links with other studies. To minimize problems in meta-analysis arising from double-counting of cases, these links were divided into three types, as shown in Additional file 2. The first involved no such double-counting, while the second included studies with minor overlap, which could not be disentangled, and which it was decided to ignore. The third type contains sets of studies which probably or definitely overlap. Here the set member containing the most valuable data (e.g. largest study size or longest follow-up) was called the 'principal study', other members being 'subsidiary studies' only considered in meta-analyses where the required $R R$ was unavailable from the principal study.

\section{Data recorded}

For each study, relevant information was entered onto a study database and a linked RR database. The study database contains a record for each study, describing relevant publications, sexes considered, age range, location, timing, length of follow-up, whether principal or subsidiary, overlaps or links with other studies, study design, populations studied, major study weaknesses, outcome definitions, numbers of cases and subjects, types of controls and matching factors used in case-control studies, confounding variables, and availability of results for each smoking index. The RR database holds the detailed results, typically containing multiple records for each study. Each record is linked to the relevant study and refers to a specific RR, recording the comparison made and the results. This record includes the outcome, the sex and the analysis type (prevalence or incidence). Smoking exposure is defined by status (ever, current or ex), product (any, cigarettes, cigarettes only) and similar information about the unexposed base. For dose-related indices, the level of exposure is recorded. The source of the RR is also recorded, as are details on adjustment variables. Results recorded include numbers of exposed and unexposed cases, and, for unadjusted results, numbers of exposed and unexposed members of the comparison group. The RR itself and its lower and upper $95 \%$ confidence limits (LCL and UCL) are always recorded, with the odds ratio chosen if available for a prevalence analysis and the relative risk (or hazard ratio if provided) for an incidence analysis. These may be as reported, or derived by various means (see below), with the method of derivation noted.

\section{Identifying which RRs to enter}

For each outcome RRs were entered relating to defined combinations of smoking index (major or dose-related), confounders adjusted for, and sex, as described below.

\section{The major smoking indices}

The intention was to enter RRs comparing current smokers, ever smokers or ex smokers with never smokers. Near-equivalent definitions were accepted when stricter definitions were unavailable, so that never smokers could include occasional smokers (or exceptionally, light smokers), while current smokers could include, and exsmokers exclude, those who quit smoking up to two years ago. If available, results were entered for five comparisons: any product vs. never any product, cigarettes vs. never any product, cigarettes only vs. never any product, cigarettes vs. never cigarettes, and cigarettes only vs. never cigarettes. Here "cigarettes" ignores whether other products (i.e. pipes and cigars) are smoked, while "cigarettes only" excludes mixed smokers.

\section{Dose-related smoking indices}

RRs were entered for five measures: amount smoked, age of starting, pack-years (cigarettes smoked per day times years of smoking, divided by 20), duration of smoking and duration of quitting. RRs were expressed relative to never smokers (or near equivalent), if available, or relative to non smokers otherwise. For duration of quitting, RRs were also expressed relative to current smokers. Further RRs were entered, restricted to smokers, and expressed relative to the level expected to have the lowest risk (e.g. lowest amount smoked, or longest time quit).

\section{Confounders adjusted for}

For prospective studies, results were entered adjusted for age and the greatest number of potential confounding variables for which results were available, and also adjusted for age only or age and the smallest number of confounders. Unadjusted results were only entered if no age-adjusted results were available. For other study types, results were entered adjusted for the greatest number of confounders, and also unadjusted (or adjusted for the smallest number of confounders). These alternative RRs are subsequently referred to as "mostadjusted" and "least-adjusted". For dose-related RRs restricted to smokers, results with "most adjustment" but without adjustment for other aspects of smoking were also entered if available. 


\section{Sex}

Results were entered for males and females separately when available, with combined sex results only entered where sex-specific results were not available.

\section{Derivation of RRs}

Adjusted RRs and their 95\% CIs were entered as provided, when available. Unadjusted RRs and CIs were calculated from their $2 \times 2$ table, using standard methods (e.g. [12]), noting any discrepancies between calculated values and those provided by the author. Sometimes the $2 \times 2$ table was constructed by summing over groups (e. g. adding current and ex smokers to obtain ever smokers) or from a percentage distribution. Various other methods were used as required to provide estimates of the RR and CI. The more commonly used methods are summarized below, fuller details being given in Additional file 1 .

Correction for zero cell. If the $2 \times 2$ table has a zero cell, 0.5 was added to each cell, and the standard formulae applied. Combining independent RRs. RRs were combined over $\ell$ strata (e.g. from a $2 \times 2 \times \ell$ table) using fixed-effect meta-analysis [13], giving an estimate adjusted for the stratifying variable. Combining nonindependent RRs. The Hamling et al method [14] was used (e.g. to derive an adjusted RR for ever smokers from available adjusted RRs for current and ex smokers, each relative to never smokers, or to combine adjusted RRs for several diseases, each relative to a single control or disease-free group). Estimating CI from crude numbers. If an adjusted RR lacked a CI or p-value but the corresponding $2 \times 2$ table was available, the CI was estimated assuming that the ratio UCL/LCL was the same as for the equivalent unadjusted RR.

\section{Data entry and checking}

Master copies of all the papers in the study file were read closely, with relevant information highlighted to facilitate checking. Where multiple papers are available for a study, a principal publication was identified, although details described only in other publications were also recorded. Preliminary calculations and data entry were carried out by one author and checked by another, and automated checks of completeness and consistency were also conducted. RR/CIs underwent validation checks $([15])$.

\section{Selecting RRs for the meta-analyses}

All meta-analyses are restricted to records with available $R R$ and CI values. The process of selecting RRs for inclusion in a meta-analysis must try to include all relevant data and to avoid double-counting. For a given analysis (e.g. of current cigarette smoking), several definitions of RR may be acceptable (e.g. cigarette smoking, or cigarette only smoking), so, for studies with multiple RRs, the one to be used is determined by an order of preference defined for the meta-analysis. Orders of preference may be required for smoking status, smoking product, the unexposed base, and extent of confounder adjustment. As the definitions of RR available may differ by sex (e.g. a study may provide RRs for any product smoking for males, but for cigarette smoking for females), the most appropriate RR is chosen within each sex. Sexes combined results are only considered where sex-specific results are not available. Similarly RRs from a subsidiary study are only used where eligible RRs are unavailable from the principal study. When multiple orders of preference are involved, the sequence of implementation may affect the selection, so preferences for the most important aspects, usually concerning smoking, are implemented first.

\section{Carrying out the meta-analyses}

Fixed-effect and random-effects meta-analyses were conducted using the methods of Fleiss and Gross [13], with heterogeneity quantified by $\mathrm{H}$, the ratio of the heterogeneity chisquared to its degrees of freedom. For all meta-analyses, Egger's test of publication bias [16] was also conducted.

A series of meta-analyses was conducted for each of the three main outcomes. For each meta-analysis conducted, combined estimates were made first for all the RRs selected, then for RRs subdivided by level of various characteristics, testing for heterogeneity between levels. These characteristics may include sex, continent, national cigarette type (blended, Virginia), start year of study, publication year, study type, lowest age included, highest age included, presence of study weakness, outcome subtype, how asthma was taken into account, use of a bronchodilator, study size (number of cases), analysis type (prevalence, onset), smoking product (any, cigarettes, cigarettes only), unexposed base (never any product, never cigarettes), smoking results available (ever smoking, current smoking, both), number of adjustment variables, whether the RR was adjusted for sex, age or for other factors, and how the RR and CI were derived. In this univariate approach, differences in fixed-effect estimates by level of a characteristic were tested for significance using an F-test which compared variation between and within levels of the characteristic considered. Additional file 1 fully defines the levels of each characteristic considered, and which characteristics are considered in each meta-analysis. It also details all the meta-analyses conducted, and describes the layout and notation used in the meta-analyses and associated forest and funnel plots. 
For each selected outcome and exposure, separate meta-analyses were conducted based on most-adjusted and least-adjusted RRs.

For the major smoking indices, four broad types of meta-analysis were conducted: A ever smoking, B current smoking, $\mathrm{C}$ ever smoking (but using current smoking RRs if ever smoking RRs are not available) and D ex smoking. In each type, RRs for the "main analysis" were selected in the following order of preference: firstly for smoking of any product vs. never smoked any product, then for smoking of cigarettes (or of cigarettes only) vs. never smoked any product, and then for smoking of cigarettes vs. never smoked cigarettes, accepting RRs vs. near-equivalents to never smokers only when RRs vs. never smokers were unavailable. A variant analysis used a different order of preference, so that RRs for cigarette smoking were preferred. In type C meta-analyses, a further variant analysis preferred RRs for current smoking to those for ever smoking. Other variant analyses restricted attention to specific subtypes of outcome (e.g. for COPD, whether the definition was based on mortality, on lung function criteria only, or on other definitions).

For the dose-related indices, meta-analyses were conducted for: $\mathrm{E}$ amount smoked, $\mathrm{F}$ age of starting to smoke, G pack-years, $\mathrm{H}$ duration of smoking, I duration of quitting compared to never smokers (or long-term ex smokers), and J duration of quitting compared to current smokers (or short-term quitters). For any measure, a study typically provides a set of non-independent RRs for each dose-category, expressed relative to a common base. To avoid double-counting only one was included in any one meta-analysis. Two approaches were adopted. The first involves specifying a scheme with a number of levels of exposure ("key values"), then carrying out meta-analyses for each level in turn. For an RR to be allocated to a key value, its dose-category has to include that key value and no other. Schemes with a few, widely spaced, key values tend to involve RRs from more studies, whereas schemes with more key values, closely spaced, involve RRs from fewer studies, but ones with dose categories more closely clustered around the key value. The key value schemes used were: $5,20 \& 45$ and $1,10,20,30,40$ \& 999 for amount smoked; 26,18 \& 14 for age of starting to smoke; $5,20 \& 45$ and $1,10,20,30$ \& 999 for pack-years; $12,7 \& 3$ and $20,12 \& 3$ for duration of quitting vs. never; and 3, $7 \& 12$ and $3,12 \& 20$ for duration of quitting vs. current (with 999 indicating an open-ended category). The second approach involves meta-analysing RRs comparing the highest vs. lowest categories of exposure. Though this approach generally includes RRs from all studies, whereas the key-value approach does not, the highest and lowest categories compared may vary markedly by study.
Meta-regression analyses of the major smoking indices For COPD and CB meta-regression analyses were also carried out using the sets of RRs selected for the main meta-analyses for ever smoking and for current smoking. Following preliminary meta-regressions (not shown), a "basic model" was fitted which included eight categorical variables (sex, continent, outcome subtype, how asthma was taken into account, smoking product, unexposed base group, adjustment for age, and adjustment for factors other than age or sex) and also midpoint age, a continuous variable estimated from the age range of the population. The significance of each of these variables was estimated by an F-test based on the increase in deviance resulting from its exclusion from the basic model. A list of secondary variables was also defined (national cigarette type, publication year, study type, presence of a study weakness, use of a bronchodilator, study size, smoking results available for the study, method of derivation of the RR and $\mathrm{CI}$ and analysis type), with the significance of adding each characteristic to the basic model estimated by an F-test based on the increase in deviance. Alternative formulations of some basic variables were also tested; see also Additional file 1.

\section{Additional analyses}

For each outcome, and for ever smoking and current smoking, pairs of corresponding RR and CI estimates within the same study for males and for females were used to carry out meta-analyses of the sex ratio. Pairs of corresponding least-adjusted or most-adjusted RRs were also identified. Unlike the sex-specific pairs, these pairs were non-independent and the variance of their ratio cannot readily be calculated. Here the numbers of pairs where the most-adjusted/least-adjusted ratio exceeded or did not exceed 1 were compared by the sign test, with separate meta-analyses also conducted for the least-adjusted and most-adjusted members. Similar methods were also used to compare non-independent pairs of RRs for current smokers of cigarettes only and for current smokers of cigarettes ignoring other products.

\section{Software}

All data entry and most statistical analysis were carried out using ROELEE version 3.1 (available from P.N.Lee Statistics and Computing Ltd, 17 Cedar Road, Sutton, Surrey SM2 5DA, UK). Some analyses were conducted using Excel 2003.

\section{Results}

\section{Studies identified}

Some 218 relevant studies were identified, based on information from 298 papers. 
For the 2,150 papers rejected, reasons are summarized in Table 1, with further details of the searching, including a flow diagram, shown in Additional file 1. Many papers had multiple reasons for rejection, the counts in Table 1 relating only to the first listed reason which applied. A Reference Manager file is available on request

\section{Table 1 Reasons for rejection of publications identified}

\begin{tabular}{|c|c|}
\hline Reason $^{a}$ & $\begin{array}{l}\text { Number of } \\
\text { publications }\end{array}$ \\
\hline IMMEDIATE REJECTS & 63 \\
\hline $\begin{array}{l}\text { Title of publication indicates it is irrelevant (abstract/ } \\
\text { paper unavailable) }\end{array}$ & 60 \\
\hline Publication could not be obtained & 3 \\
\hline PUBLICATION DOES NOT PROVIDE ORIGINAL DATA & 430 \\
\hline $\begin{array}{l}\text { Results the same as or superseded by another } \\
\text { publication }\end{array}$ & 16 \\
\hline $\begin{array}{l}\text { Review (including guideline, handout, lecture, } \\
\text { bibliography, meta-analysis) }\end{array}$ & 329 \\
\hline Editorial & 31 \\
\hline Comment, letter, interview or news article & 47 \\
\hline Publication is a theoretical modelling exercise & 7 \\
\hline STUDY POPULATION INAPPOPRIATE & 343 \\
\hline Study of children or adolescents & 30 \\
\hline Animal study & 5 \\
\hline $\begin{array}{l}\text { Study in population at high risk of respiratory disease, } \\
\text { such as risky occupations }\end{array}$ & 71 \\
\hline Study of alpha-1antitrypsin deficient subjects & 39 \\
\hline $\begin{array}{l}\text { Study of subjects with other coexisting diseases or } \\
\text { conditions }\end{array}$ & 105 \\
\hline Study of atypical populations & 7 \\
\hline Subjects selected on smoking habits & 61 \\
\hline $\begin{array}{l}\text { Study of symptom-free or symptom-restricted } \\
\text { populations }\end{array}$ & 25 \\
\hline STUDY DESIGN INAPPROPRIATE & 323 \\
\hline Not a case-control, prospective or cross-sectional study & 84 \\
\hline Study of cases only & 216 \\
\hline Control group not appropriate & 9 \\
\hline Selection of subjects not clear & 14 \\
\hline OUTCOME INAPPROPRIATE & 566 \\
\hline Outcome not relevant & 557 \\
\hline Study of undiagnosed disease & 4 \\
\hline Study of disease exacerbation & 5 \\
\hline USEFUL RESULTS BY SMOKING UNAVAILABLE & 425 \\
\hline Never smokers not considered & 36 \\
\hline No relevant results by smoking & 304 \\
\hline $\begin{array}{l}\text { Comparisons with never smokers and ex-smokers } \\
\text { combined }\end{array}$ & 17 \\
\hline Study of smokers of unusual cigarettes (e.g. chuttas) & 2 \\
\hline Relative risks not calculable & 61 \\
\hline $\begin{array}{l}\text { Relative risks adjusted for symptoms or precursors of } \\
\text { disease }\end{array}$ & 5 \\
\hline Total rejected & 2150 \\
\hline
\end{tabular}

${ }^{a}$ Where publications had more than one reason for rejection, the publication is counted under the first relevant reason listed which, for each rejected publication, gives its reference and the reasons for rejection.

Table 2 presents selected details of the 218 studies while Table 3 gives the distribution of their major characteristics. Additional file 2 gives fuller descriptions of the studies, including overlapping and linked studies, medical and other exclusions, detailed definitions of disease outcomes, and fuller distributions.

Of the 218 studies, 193 are classified as principal, 20 (10.4\%) of these being case-control studies, 39 (22.7\%) prospective, and $134(69.4 \%)$ cross-sectional. The other 25 studies are classified as subsidiary. Ninety-three principal studies are of COPD only, 63 of CB only, nine of emphysema only, with 28 providing results for multiple outcomes. In total, information is available on COPD for 133 studies (116 principal), CB for 101 (87 principal) and emphysema for 28 (26 principal). Of the principal studies, only $9(10.3 \%)$ are prospective for $\mathrm{CB}$, compared with 35 (30.2\%) for COPD and 7 (26.9\%) for emphysema. There are no case-control studies for emphysema.

Of the 193 principal studies, 145 (75.1\%) provide results for both sexes, 42 (21.8\%) for males only, and six (3.1\%) for females only. Ninety-six (49.7\%) of the studies include subjects under 30 (at baseline for prospective studies), while only $24(12.4 \%)$ are restricted to subjects aged 50 or more. Subjects aged 80 or more are included by $103(53.6 \%)$, while only $20(10.3 \%)$ are restricted to subjects aged 60 or less. Fifty-six (29.0\%) principal studies were conducted in USA or Canada, with $32(16.6 \%)$ in Scandinavia, 32 (16.6\%) in Asia, 23 (11.9\%) in the UK, 17 (8.8\%) in Western Europe, 14 (7.3\%) in other parts of Europe, eight (4.1\%) in South or Central America and seven (3.6\%) elsewhere. Four (2.1\%) were carried out in more than one of these areas. Of the 159 principal studies where the start year is given, $76(47.7 \%)$ started before 1980. For 26 (13.5\%) of the 193 studies a major study weakness is noted. Most commonly this is a failure to clarify, or to state at all, how study subjects were selected (studies ALESSA, ANDER3, COCCI, ITABAS, MOLLER, SHIMUR, ZIETKO). Other more commonly occurring weaknesses include use of unrepresentative samples which oversampled smokers (DEJONG, DETORR, JENSEN), those with respiratory disease (VOLLM1, VOLLM2) or those with occupational exposure (PETO, PRATT), and the use of controls that systematically differ from cases and controls in various ways (BROGGE, DEAN1, LUNDB2, STERLI). These weaknesses are described more fully in the footnotes to Table 2 .

Most principal studies provide some results compared to never smokers, 146 (75.6\%) for current smokers, 134 $(69.4 \%)$ for ex smokers and $158(81.8 \%)$ for ever smokers. Dose-response data are commonly available by amount smoked (77 studies, 39.9\%) and by pack-years 
Table 2 Selected details of the 218 studies of COPD, CB and/or emphysema

\begin{tabular}{|c|c|c|c|c|c|c|c|}
\hline $\begin{array}{l}\text { Study REF } \\
\text { [refs] }\end{array}$ & $\begin{array}{l}\text { Study } \\
\text { type }^{a}\end{array}$ & Country & Years $^{b}$ & Population $^{c}$ & $\begin{array}{l}\text { Outcome } \\
(s)^{d}\end{array}$ & $\begin{array}{l}\text { Study group (if } \\
\text { Subsid) }\end{array}$ & Weakness $^{f}$ \\
\hline ALDERS $[26,27]$ & CCh & UK/England & 1977-82 & & $C B$ & & No \\
\hline ALESSA [28] & CCp & Italy & 1992-93 & & COPD & & Yes \\
\hline AMIGO [29] & CCh & Chile & 2001-03 & & COPD & & No \\
\hline ANDER1 [30] & CS & Canada & 1963 & & COPD, CB & & No \\
\hline ANDER2 [31] & CS & USA & (ca 1964?) & & EM & & No \\
\hline ANDER3 $[32,33]$ & CCp & Poland & (ca 1997?) & & COPD & & Yes \\
\hline AUERBA $[34,35]$ & CS & USA & 1963-70 & & EM & & No \\
\hline BANG [36] & CS & USA & $1982-84$ & hispanic & $\mathrm{CB}$ & & No \\
\hline BECK1 [37] & $\mathrm{CS}$ & USA & 1972-73 & & $C B$ & & No \\
\hline BECK2 [37] & $P$ & USA & 1972-73/1978 & & $\mathrm{CB}$ & & No \\
\hline BEDNAR [38] & $\mathrm{CS}$ & Poland & 2000-02 & & COPD & & No \\
\hline BEST $[39,40]$ & $P$ & Canada & $1955-56 / 1962$ & military veterans & $\begin{array}{l}\text { COPD, CB, } \\
\text { EM }\end{array}$ & & No \\
\hline BJORNS [41] & $\mathrm{CS}$ & Sweden & 1990 & & $\mathrm{CB}$ & & No \\
\hline BROGGE [42] & $\mathrm{CCm}$ & Norway & 2003 & & COPD & JOHANN & Yes \\
\hline BROWN [43] & $\mathrm{CS}$ & UK/England & 1956 & & $C B$ & & No \\
\hline CERVER [44] & $\mathrm{CS}$ & Italy & 1998-00 & & $C B$ & & No \\
\hline CHAPMA [45] & $\mathrm{CS}$ & USA & 1976 & parents & $\mathrm{CB}$ & HOUSE & No \\
\hline CHEN1 [46] & $P$ & China & 1972-78/1993 & workers at 11 factories & COPD & & No \\
\hline CHEN2 [47] & CS & Canada & 1994-95 & household members & COPD & & No \\
\hline CHEN3 [48] & CS & Canada & 2000-01 & household members & COPD & & Yes \\
\hline CHENG [49] & CS & China & 1992 & & COPD & & No \\
\hline CLEMEN [50] & $P$ & Belgium & $1960-* / 1975$ & Air Force personnel & COPD & & No \\
\hline COATES [51] & CS & USA & 1962 & Post Office employees & $C B$ & & No \\
\hline $\mathrm{COCCl}[52]$ & CCp & Italy & (ca 2000?) & & COPD & & Yes \\
\hline COLLEG $[53,54]$ & $\mathrm{CS}$ & UK/GB & (ca 1960?) & & $\mathrm{CB}$ & & No \\
\hline DEAN1 $[55,56]$ & CCp & UK/England & 1969-73 & & COPD & & Yes \\
\hline DEAN2 [57] & $\mathrm{CS}$ & UK/GB & 1972 & & $\mathrm{CB}$ & & No \\
\hline DEANE [58] & CS & USA & 1963 & telephone company employees & $\mathrm{CB}$ & & No \\
\hline DEJONG [59] & $\mathrm{CS}$ & USA & (ca 2003?) & & COPD & & Yes \\
\hline DEMARC [60-62] & CS & Multi-Europe & 1991-93 & & COPD, CB & & No \\
\hline DETORR [63] & CS & Spain & 2001-03 & patients & COPD & & Yes \\
\hline DICKIN [64] & CS & UK/England & (ca 1997?) & & COPD & & No \\
\hline DOLL1 [65-67] & $P$ & UK & 1951/1991 & doctors & COPD, CB & & No \\
\hline DOLL2 $[66,68]$ & $P$ & UK & 1951/1973 & doctors & COPD, CB & & No \\
\hline DONTA1 [69] & CS & Greece & 1960 & & COPD & JACOBS & Yes \\
\hline DONTA2 [69] & $P$ & Greece & 1960/1970 & & $C B, E M$ & JACOBS & Yes \\
\hline DOPICO [70] & CS & USA & (ca 1982?) & employed $^{g}$ & $\mathrm{CB}$ & & No \\
\hline EHRLIC [71] & CS & South Africa & 1998 & household members & CB & & No \\
\hline EKBERG [72] & CS & Sweden & 1974-92 & & COPD & & No \\
\hline ENRIGH [73] & CS & USA & 1989-90 & health insurance members & $C B, E M$ & & No \\
\hline ENSTRO [74] & $P$ & USA & 1960/1998 & household members & COPD & HAMMO2 & No \\
\hline FERRI1 [54,75-77] & CS & USA & 1961 & household members & COPD, CB & FERRI2 & No \\
\hline FERRI2 [75,78] & CS & USA & 1967 & $\begin{array}{l}\text { household members, long term } \\
\text { residents }\end{array}$ & COPD & & No \\
\hline FERRI3 [79] & CS & USA & 1973 & $\begin{array}{l}\text { household members, long term } \\
\text { residents }\end{array}$ & COPD & FERRI2 & No \\
\hline FIDAN [80] & CS & Turkey & 2000-01 & coffeehouse or shop workers & COPD & & No \\
\hline FINKLE [81] & CS & USA & 1969-70 & military recruits & CB & & No \\
\hline FLETCH [82] & CS & UK & $1956-57$ & employed $^{9}$ & $C B$ & & No \\
\hline FORAST [83] & CS & USA & 1993-94 & & COPD & & Yes \\
\hline
\end{tabular}


Table 2 Selected details of the 218 studies of COPD, CB and/or emphysema (Continued)

\begin{tabular}{|c|c|c|c|c|c|c|c|}
\hline FOXMAN [84] & CS & USA & (ca 1981?) & & $C B$ & & No \\
\hline FUKUCH [85] & CS & Japan & 2000 & household members & COPD & & No \\
\hline GEIJER [86] & $P$ & Netherlands & 1998/2003 & & COPD & & No \\
\hline GODTFR $[18,87]$ & $P$ & Denmark & 1964-93/1997 & & COPD & & No \\
\hline GOLDBE [88] & CS & USA & 1970 & parents & $\mathrm{CB}$ & & No \\
\hline GULSVI [89-91] & CS & Norway & $1972-74$ & & COPD, EM & & No \\
\hline HAENSZ [92] & CS & Norway & 1964 & mixed $^{\text {h }}$ & $\mathrm{CB}$ & & No \\
\hline $\begin{array}{l}\text { HAMMO2 } \\
\text { [93-97] }\end{array}$ & $P$ & USA & 1959-60/1965 & household members & COPD, EM & & No \\
\hline HARDIE [98] & CS & Norway & 1998-99 & & $\begin{array}{l}\text { COPD, CB, } \\
\text { EM }\end{array}$ & & No \\
\hline HARIKK [99] & $\mathrm{P}$ & USA & $\begin{array}{l}1962-* /(c a \\
2000 ?)\end{array}$ & & COPD & & No \\
\hline HARRIS [100] & CS & Nigeria & (ca 1992?) & soldiers & $\mathrm{CB}$ & & No \\
\hline HAWTHO [101] & $P$ & UK/Scotland & 1965-75/1977 & mixed $^{\text {h }}$ & COPD, CB & TANG & Yes \\
\hline HAYES [102] & $\mathrm{CS}$ & USA & 1970 & parents & $\mathrm{CB}$ & & No \\
\hline HEDMAN [103] & CS & Finland & 1996 & & COPD & & No \\
\hline HIGGI2 [104] & $\mathrm{CS}$ & UK/Wales & 1956 & & $\mathrm{CB}$ & PETO & No \\
\hline HIGGI3 [105] & CS & UK/Scotland & 1956 & & $\mathrm{CB}$ & & No \\
\hline HIGGI4 [106,107] & $P$ & USA & 1962-79/1987 & & COPD & & Yes \\
\hline HIGGI6 [108,109] & $\mathrm{CS}$ & USA & $1962-65$ & & $\mathrm{CB}$ & $\mathrm{HIGGI4}$ & No \\
\hline $\begin{array}{l}\text { HIRAYA } \\
{[110,111]}\end{array}$ & $P$ & Japan & 1965/1982 & & $C B, E M$ & & No \\
\hline $\mathrm{HO}[112,113]$ & $\mathrm{CS}$ & Hong Kong & 1991 & long term residents & $\begin{array}{l}\text { COPD, CB, } \\
\text { EM }\end{array}$ & & No \\
\hline HOLLA2 [114] & CS & USA & 1962 & telephone company employees & $C B$ & & No \\
\hline $\begin{array}{l}\text { HOLLNA } \\
{[115,116]}\end{array}$ & $\mathrm{CS}$ & Denmark & 1976-77 & & $C B$ & & No \\
\hline HOUSE [117] & CS & USA & 1970 & parents & $\mathrm{CB}$ & & No \\
\hline $\begin{array}{l}\text { HOZAWA } \\
\text { [118-120] }\end{array}$ & CS & USA & 1987-89 & & COPD, EM & & No \\
\hline HRUBEC [121] & $\mathrm{CS}$ & USA & (ca 1972?) & veterans and twins & $\mathrm{CB}$ & & No \\
\hline HUCHON [122] & CS & France & (ca 2001?) & household members & $C B$ & & No \\
\hline HUHTI1 [123] & CS & Finland & 1961 & & $\begin{array}{l}\text { COPD, CB, } \\
\text { EM }\end{array}$ & & No \\
\hline HUHTI2 [124] & CS & Finland & 1971 & long term residents & COPD, CB & HUHTI1 & No \\
\hline HUHTI3 [125] & $\mathrm{CS}$ & Finland & $1968-70$ & & COPD, CB & & No \\
\hline ITABAS [126] & CCp & Japan & (ca 1989?) & & COPD & & Yes \\
\hline JACOBS [127] & $P$ & Multi-Europe & $\begin{array}{l}1957-64 /(\mathrm{ca} \\
1989 ?)\end{array}$ & & COPD & & No \\
\hline JAENDI [128] & $\mathrm{CS}$ & Spain & 2001-02 & patients & COPD & & Yes \\
\hline JENSEN [129] & CS & Denmark & (ca 1996?) & patients & $\mathrm{CB}$ & & Yes \\
\hline JINDA2 [130] & CS & India & (ca 2004?) & household members & $\mathrm{CB}$ & & No \\
\hline $\begin{array}{l}\text { JOHANN } \\
\text { [131-134] }\end{array}$ & CS & Norway & 1996-97 & long term residents & COPD & & No \\
\hline JOSHI [135] & CS & India & (ca 1974?) & employed $^{9}$ & $C B$ & & No \\
\hline JOUSI1 [136] & CS & Finland & 1972-77 & & $C B$ & & No \\
\hline KACHEL [137] & CS & Poland & (ca 2002?) & workers at five factories & COPD & & No \\
\hline KAHN [138-144] & $P$ & USA & $1954-57 / 1980$ & military veterans & $\begin{array}{l}\text { COPD, CB, } \\
\text { EM }\end{array}$ & & No \\
\hline KAHN2 [138] & $P$ & USA & $1954-57 / 1962$ & military veterans & $\begin{array}{l}\text { COPD, CB, } \\
\text { EM }\end{array}$ & KAHN & No \\
\hline KARAKA [145] & NCCp & Greece & */1996 & & COPD & VINEIS & No \\
\hline KATANC [146] & CS & USA & 1997-98 & mixed $^{\text {h }}$ & COPD & & No \\
\hline KATO [147] & CS & Japan & 1985 & & $\mathrm{CB}$ & & No \\
\hline
\end{tabular}


Table 2 Selected details of the 218 studies of COPD, CB and/or emphysema (Continued)

\begin{tabular}{|c|c|c|c|c|c|c|c|}
\hline KHOURY [148] & CS & USA & (1970s) & mixed $^{\text {h }}$ & \multicolumn{2}{|l|}{ COPD } & Yes \\
\hline KIM [149] & CS & Korea & 2001-02 & household members & \multicolumn{2}{|l|}{ COPD } & No \\
\hline KIRAZ [150] & CS & Turkey & 1999 & mixed $^{\text {h }}$ & \multicolumn{2}{|c|}{ COPD, CB } & No \\
\hline KLAYTO [151] & CS & USA & (ca 1974?) & employed $^{g}$ & \multicolumn{2}{|l|}{ COPD } & Yes \\
\hline KOJIMA [152] & CS & Japan & 2001-02 & & \multicolumn{2}{|l|}{ COPD } & No \\
\hline KOTAN1 [153] & CS & Finland & 1995-96 & & \multicolumn{2}{|l|}{$C B$} & No \\
\hline KOTAN2 [154] & CS & Finland & 1996-97 & & COPD & KOTAN1 & No \\
\hline KRZYZA [155] & $P$ & Poland & 1968/1981 & & \multicolumn{2}{|l|}{ COPD } & No \\
\hline KUBIK [156] & CS & Czechoslovakia & 1972 & & \multicolumn{2}{|l|}{$\mathrm{CB}$} & Yes \\
\hline KULLER [157] & P & USA & 1972-74/1980 & volunteers & \multicolumn{2}{|l|}{ COPD } & No \\
\hline LAI [158] & CS & China & 2001-03 & & \multicolumn{2}{|l|}{ COPD } & No \\
\hline LAM1 [159] & $P$ & China & 1976/1996 & employed $^{9}$ & \multicolumn{2}{|l|}{ COPD } & No \\
\hline LAM2 [160,161] & CS & China & 1987 & military veterans & \multicolumn{2}{|l|}{ COPD } & No \\
\hline LAM3 [160-162] & $P$ & China & 1987/2005 & military veterans & \multicolumn{2}{|l|}{ COPD } & No \\
\hline $\begin{array}{l}\text { LAMBER } \\
{[163,164]}\end{array}$ & CS & UK & 1965 & household members & $\mathrm{CB}$ & TODD & No \\
\hline LANGE [165-169] & $P$ & Denmark & 1976-78/1989 & & COPD & GODTFR & No \\
\hline LANGE2 [170] & CS & Denmark & 1991-94 & & $\mathrm{CB}$ & VESTBO & No \\
\hline LANGHA [19] & CS & Norway & 1995-97 & & $\mathrm{CB}$ & & No \\
\hline LAVECC [171] & CS & Italy & 1983 & household members & $C B, E M$ & & No \\
\hline $\begin{array}{l}\text { LEBOWI } \\
{[172-177]}\end{array}$ & CS & USA & $1972-73$ & household members & $\begin{array}{l}\text { COPD, } \\
\text { EM }\end{array}$ & & No \\
\hline LEE $[164,178]$ & $P$ & UK & 1964-65/1977 & siblings of migrants & COPD & & No \\
\hline LIAW [179] & $P$ & Taiwan & 1982-86/1993 & volunteers & COPD & WEN & No \\
\hline LINDBE [180] & $P$ & Sweden & 1996/2003 & long term residents & COPD & & No \\
\hline LINDST [181] & CS & Europe & (ca 1998?) & & COPD, C & & No \\
\hline LIU1 [182] & $\mathrm{cCd}$ & China & 1986-91 & & COPD & & No \\
\hline LIU2 [183,184] & CS & China & 2002-03 & & COPD & & No \\
\hline $\begin{array}{l}\text { LUNDB1 } \\
{[185,186]}\end{array}$ & CS & Sweden & 1996-97 & long term residents & COPD & & No \\
\hline $\begin{array}{l}\text { LUNDB2 } \\
\text { [187-189] }\end{array}$ & CCp & Sweden & 1986 & & $C B$ & LUNDB1 & Yes \\
\hline MADOR [190] & $\mathrm{CCh}$ & USA & (ca 2002?) & military veterans & COPD & & No \\
\hline MAGNUS [191] & CS & Iceland & 1993 & & $\mathrm{CB}$ & & No \\
\hline MANFRE [192] & CS & Canada & 1978-79 & & $\mathrm{CB}$ & & No \\
\hline $\begin{array}{l}\text { MANNI1 } \\
\text { [193-198] }\end{array}$ & CS & USA & 1988-94 & & COPD & & No \\
\hline $\begin{array}{l}\text { MANNI2 } \\
{[199,200]}\end{array}$ & CS & USA & $1971-75$ & household members & COPD & & No \\
\hline MANNI3 [199] & $P$ & USA & 1971-75/1992 & household members & COPD & & No \\
\hline MARAN1 [201] & CS & Thailand & 1998 & & COPD & & Yes \\
\hline MARAN2 [201] & $P$ & Thailand & 1998/1999 & & COPD & & Yes \\
\hline MARCUS [202] & $P$ & USA & 1965-68/1984 & Japanese ancestry & COPD & & No \\
\hline MATHES [203] & CCp & Australia & (ca 2005?) & & COPD & & No \\
\hline MELLST [204] & CS & Sweden & 1971-77 & & $\mathrm{CB}$ & & No \\
\hline MENEZ1 [205] & CS & Brazil & 1990 & household members & $\mathrm{CB}$ & & No \\
\hline $\begin{array}{l}\text { MENEZ2 } \\
{[206,207]}\end{array}$ & CS & Brazil & 2003 & household members & COPD & & No \\
\hline MENEZ3 [206] & CS & Chile & 2003 & household members & COPD & & No \\
\hline MENEZ4 [206] & CS & Mexico & 2003 & household members & COPD & & No \\
\hline MENEZ5 [206] & CS & Uruguay & 2003 & household members & COPD & & No \\
\hline MENEZ6 [206] & CS & Venezuela & 2003 & household members & COPD & & No \\
\hline MEREN [208] & CS & Estonia & 1995-96 & & $C B$ & & No \\
\hline MILLER [209] & CS & USA & 1978 & household members & $C B, E M$ & & No \\
\hline
\end{tabular}


Table 2 Selected details of the 218 studies of COPD, CB and/or emphysema (Continued)

\begin{tabular}{|c|c|c|c|c|c|c|c|}
\hline MILNE $[210,211]$ & CS & UK/Scotland & $1968-70$ & & $C B$ & & No \\
\hline MOLLER [212] & $\mathrm{CCp}$ & Germany & (ca 1999?) & & $C B$ & & Yes \\
\hline $\begin{array}{l}\text { MONTNE } \\
{[213-215]}\end{array}$ & CS & Sweden & 1992 & & COPD & & No \\
\hline MUELLE [216] & CS & USA & 1967 & household members & COPD, CB & & No \\
\hline NAWA [217] & CS & Japan & 1998-00 & mixed $^{h}$ & EM & & No \\
\hline NEJJAR [218] & CS & France & 1991 & household members & $\mathrm{CB}$ & & No \\
\hline NIEPSU [219] & CS & Poland & 2001 & & COPD & & Yes \\
\hline $\begin{array}{l}\text { NIHLEN } \\
{[220,221]}\end{array}$ & $P$ & Sweden & 1992/2000 & long term residents & COPD & & No \\
\hline $\begin{array}{l}\text { NILSSO } \\
{[222-224]}\end{array}$ & $P$ & Sweden & 1963/1996 & & COPD & & No \\
\hline OGILVI [225] & CCp & UK/England & $1955-56$ & household members & $\mathrm{CB}$ & & No \\
\hline OMORI [226] & CS & Japan & (ca 2004?) & & EM & & Yes \\
\hline OSWAL1 [227] & $\mathrm{CCh}$ & UK/England & 1951-53 & mixed $^{\text {h }}$ & $\mathrm{CB}$ & & No \\
\hline OSWAL2 [228] & CS & UK/England & $1954-55$ & civil servants & $C B$ & & Yes \\
\hline $\begin{array}{l}\text { PANDEY } \\
{[229,230]}\end{array}$ & CS & Nepal & 1979-80 & & $C B$ & & No \\
\hline PEAT $[231,232]$ & P & Australia & 1966-75/1984 & & COPD & & No \\
\hline PELKON [233] & P & Finland & 1959/2000 & & COPD, CB & JACOBS & No \\
\hline PEREZP [234] & $\mathrm{CCm}$ & Mexico & 1992-94 & & $\mathrm{COPD}, \mathrm{CB}$ & & No \\
\hline PETO [235] & $\mathrm{P}$ & $\begin{array}{l}\text { UK/England and } \\
\text { Wales }\end{array}$ & 1954-61/1981 & mixed $^{\text {h }}$ & COPD & & Yes \\
\hline PRATT [236] & CS & USA & (ca 1978?) & military veterans & EM & & Yes \\
\hline PRICE $[237,238]$ & $\mathrm{CS}$ & UK & (ca 2004?) & & COPD & & No \\
\hline REID [239] & CS & USA & $1962-63$ & mixed $^{\text {h }}$ & $\mathrm{CB}$ & & No \\
\hline RENWIC [240] & CS & UK/England & 1992-94 & & COPD & & No \\
\hline RICCIO [241] & CS & Italy & 2002 & patients & COPD & & Yes \\
\hline $\begin{array}{l}\text { RIMING } \\
{[242,243]}\end{array}$ & CS & UK/England & 1970 & volunteers & $C B$ & & No \\
\hline RYDER [244] & CS & UK/Wales & (ca 1969?) & & EM & & No \\
\hline $\begin{array}{l}\text { SARGEA } \\
{[245,246]}\end{array}$ & CS & UK/England & 1993-96 & & COPD & VINEIS & No \\
\hline $\begin{array}{l}\text { SAWICK } \\
{[247,248]}\end{array}$ & CS & Poland & 1968 & & COPD, CB & & No \\
\hline $\begin{array}{l}\text { SCHWAR } \\
{[249,250]}\end{array}$ & CS & USA & 1976-80 & household members & $C B$ & & No \\
\hline SHAHAB [251] & CS & England & 2001 & & COPD & & No \\
\hline SHARP $[252,253]$ & CS & USA & 1960-61 & employed $^{9}$ & $C B$ & & No \\
\hline SHIMUR [254] & $\mathrm{CCh}$ & Japan & (ca 1994?) & & $C B$ & & Yes \\
\hline SHIN [255] & CS & Korea & 1999-00 & household members & COPD & & No \\
\hline SICHLE [256] & CS & Greece & 2000-01 & & COPD & & No \\
\hline SILVA [257-260] & $P$ & USA & $\begin{array}{l}19722^{*} /(\mathrm{ca} \\
1992 ?)\end{array}$ & household members & $\begin{array}{l}\text { COPD, CB, } \\
\text { EM }\end{array}$ & & No \\
\hline $\begin{array}{l}\text { SOBRAD } \\
{[261-264]}\end{array}$ & CS & Spain & 1996-97 & & $\mathrm{CB}$ & & No \\
\hline SPEIZE [265] & $P$ & USA & 1974-77/1986 & household members & COPD & & No \\
\hline STERLI $[266,267]$ & CCp & USA & 1986-87 & & COPD & & Yes \\
\hline STJERN [268] & CS & Sweden & 1981 & & $C B$ & & No \\
\hline STROM [269] & CS & Sweden & $1982-83$ & long term residents & COPD & & No \\
\hline SUADIC [270] & CS & Denmark & $1985-86$ & employed $^{9}$ & $C B$ & & No \\
\hline SUTINE [271] & CS & Finland & $1971-72$ & & EM & & No \\
\hline TAGER [272] & CS & USA & 1973-74 & & COPD & & No \\
\hline TAGER2 [273] & CS & USA & 1973-74 & household members & CB & & Yes \\
\hline TANG [274] & $P$ & UK & $1967-82 /^{*}$ & mixed $^{h}$ & COPD & & No \\
\hline
\end{tabular}


Table 2 Selected details of the 218 studies of COPD, CB and/or emphysema (Continued)

\begin{tabular}{|c|c|c|c|c|c|c|c|}
\hline $\begin{array}{l}\text { THUN } \\
{[96,97,275,276]}\end{array}$ & $P$ & USA & 1982/1988 & household members & COPD & & No \\
\hline TODD $[164,178]$ & $P$ & UK & 1965-66/1977 & household members & COPD & & No \\
\hline TROISI [277] & $P$ & USA & 1980/1990 & nurses & $\mathrm{CB}$ & & No \\
\hline $\begin{array}{l}\text { TRUPIN } \\
{[278,279]}\end{array}$ & CS & USA & 2001 & telephone subscribers & COPD & & No \\
\hline TSUSHI [280] & CS & Japan & 2003-04 & volunteers & COPD & & No \\
\hline TVERDA [281] & $P$ & Norway & 1972-78/1988 & & COPD & & Yes \\
\hline URRUTI [282] & CS & Spain & (ca 2004?) & & $\mathrm{CB}$ & DEMARC & No \\
\hline $\begin{array}{l}\text { VESTBO } \\
{[168,283]}\end{array}$ & CS & Denmark & $1976-78$ & & COPD & & No \\
\hline VIEGI1 [284] & CS & Italy & 1980-82 & household members & $\mathrm{CB}$ & & No \\
\hline VIEGI2 [285] & CS & Italy & 1988-91 & & COPD & & No \\
\hline VIKGRE [286-288] & $P$ & Sweden & 1994-95/2001 & long term residents & EM & & No \\
\hline VINEIS [289] & $P$ & Europe & $\begin{array}{l}\text { 1993-98/(ca } \\
2003 ?)\end{array}$ & volunteers & COPD & & No \\
\hline VOLLM1 [290] & CS & USA & $1971-72$ & volunteers & COPD & VOLLM2 & Yes \\
\hline VOLLM2 [290] & $P$ & USA & 1971-72/1982 & volunteers & COPD & & Yes \\
\hline VONHER [291] & CS & Finland & $1978-80$ & & COPD & & No \\
\hline $\begin{array}{l}\text { WAGEN2 } \\
\text { [292-294] }\end{array}$ & CCp & Netherlands & 2001 & employed $^{9}$ & $C B$ & & No \\
\hline WALD [295] & $P$ & UK/England & 1975-82/1993 & professional/business men & COPD & TANG & Yes \\
\hline WANG2 [296] & CS & Japan & $1996-98$ & volunteers & EM & & No \\
\hline WATSON [297] & CCp & UK & (ca 2000?) & & COPD & & No \\
\hline WEISS [298] & CS & USA & 1961 & volunteers & COPD, EM & & No \\
\hline WEN [299] & $P$ & Taiwan & $1982-92 / 2000$ & mixed $^{\text {h }}$ & $\begin{array}{l}\text { COPD, CB, } \\
\text { EM }\end{array}$ & & No \\
\hline WIG [300] & CS & India & (ca 1963?) & household members & $\mathrm{CB}$ & & Yes \\
\hline WILHEL [301] & CS & Sweden & 1967 & & $\mathrm{CB}$ & & No \\
\hline WILSO1 [302] & CS & Australia & 2000 & household members & COPD & & No \\
\hline WILSO2 [303] & CS & Australia & 1998 & household members & $\mathrm{CB}, \mathrm{EM}$ & & No \\
\hline $\begin{array}{l}\text { WOJTYN } \\
{[304,305]}\end{array}$ & CS & Poland & $1968-73$ & & COPD, CB & SAWICK & No \\
\hline WOODS [306] & CS & Australia & (ca 1998?) & & $\mathrm{CB}$ & & No \\
\hline WOOLF [307] & CS & Canada & (ca 1970-73?) & employed $^{9}$ & $\mathrm{CB}$ & & No \\
\hline $\mathrm{XIAO}$ [308] & CCp & China & (ca 2003?) & & COPD & & No \\
\hline$X U$ [309] & CS & China & 2000-01 & long term residents & COPD & & No \\
\hline $\begin{array}{l}\text { YAMAGU } \\
{[20,310]}\end{array}$ & CS & China & 1986 & long term residents & COPD, CB & & No \\
\hline YUAN [311] & $P$ & China & 1986-89/1993 & & COPD & & No \\
\hline ZIELI1 [312-314] & CS & Poland & 1999 & volunteers & COPD & & No \\
\hline ZIELI2 [315-317] & CS & Poland & 2000-03 & volunteers & COPD & & No \\
\hline ZIETKO [318] & CCp & Poland & (ca 2003?) & & COPD & & Yes \\
\hline ZOIA [319] & CS & Italy & (ca 1993?) & & $\mathrm{CB}$ & & No \\
\hline
\end{tabular}

${ }^{\mathrm{a}} \mathrm{CS}=$ cross-sectional, $\mathrm{P}=$ prospective, $\mathrm{CC}=$ case control, NCC = nested case-control, $\mathrm{h}=$ hospital or clinic controls, $\mathrm{p}=$ population controls, $\mathrm{d}=\mathrm{decedent}$ controls, $\mathrm{m}=$ mixed controls.

$b_{*}=$ unknown. Values in brackets are approximate, based on one year before first publication. For prospective studies, baseline year(s)/final follow-up year. Results refer to the full follow-up period except:

DOLL1, DOLL2 CB, to 1961

HIRAYA Emphysema (except by amount smoked), to 1978

KAHN Current smoking by amount smoked, to 1970

NILSSO Smoking of any product, to 1979

THUN Ex and ever smoking, to 1986

' Unless shown otherwise in this column, the study specified no major inclusion criteria.

${ }^{d}$ COPD = chronic obstructive lung disease, $C B=$ chronic bronchitis, $E M=$ emphysema

e For subsidiary studies, this column shows the relevant principal study. 
${ }^{f}$ Weakness identified, including studies where the base for comparison of the major smoking indices was not strictly never smokers:

ALESSA Small clinical study, not stated how subjects selected

ANDER3 Small clinical study, not clear how controls were selected

BROGGE More cases than controls were drawn from hospital sample (with hospitalisation for COPD in last 5 years) and their average age was 3.5 years older CHEN3 Analysis combines current smokers with those who gave up in last 5 years, and omits those who started smoking before age 13 or after age 22 COCCI Small clinical study, not stated how cases and controls were selected

DEAN1 Cases occurred in 1969-72 while information on controls was collected in 1973. Cases were population sample but controls were household members only

DEJONG Non-representative convenience sample particularly aimed at smokers

DETORR Subjects were volunteers, invited from all smokers attending wards or clinics, so likely to have concomitant disease

DONTA1 Inclusion of various lung diseases other than COPD in study endpoint, exclusion of subjects who died, emigrated or made dramatic changes to their smoking habits during follow-up

DONTA2 Exclusion of subjects who died, emigrated or made dramatic changes to their smoking habits during follow-up

FORAST Cases without symptoms in the last year were excluded

HAWTHO Base for comparison includes smokers of up to 5 cigarettes per day

HIGGI4 Because of inadequate detail in the report and use of differing age groups in different tables, estimates are rather speculative

ITABAS Small clinical study, not stated how cases and controls were selected

JAENDI Study population were those who visited their primary care physician, so may have been less healthy than the general population. Some attempts were made to contact patients who did not visit their physician during the study period, but it is unclear if they were then included in the study. It is not clear why only $7 \%$ of subjects were age $65+$

JENSEN All subjects were participants in smoking cessation programme

KHOURY $13 \%$ of sample were 1 st degree relatives of COPD cases, and a further $3 \%$ were 1 st degree relatives of lung cancer cases

KLAYTO Base for comparison includes smokers of up to 5 pack-years

KUBIK Base for comparison includes smokers of up to 3 cigarettes per day

LUNDB2 A few subjects were analysed as controls (as determined at the start of the study) even if diagnosed with CB or asthma at the second phase of the study when the diagnosis category of the cases was determined

MARAN1 Base for comparison includes smokers of up to 0.5 pack-years

MARAN2 Base for comparison includes smokers of up to 0.5 pack-years

MOLLER Small clinical study, not clear how subjects were selected

NIEPSU Numbers of smokers not given for subset of participants undergoing spirometry (74\%), therefore estimated using same proportions as whole study sample

OMORI Different diagnostic techniques used for smokers

OSWAL2 Base for comparison includes smokers of up to 5 cigarettes per day or who had smoked for less than 5 years

PETO Three of the samples were drawn from mining areas with over $60 \%$ miners or other dusty jobs, implying about $40 \%$ of the overall sample were occupationally exposed.

PRATT Study contained small number of subjects who were cotton or tobacco farmers or who worked in tobacco factory

RICCIO Subjects were recruited through a respiratory clinic but it is not stated whether they all had respiratory conditions. The definition of a smoker seems implausible

SHIMUR Small autopsy study, not clear how subjects were selected

STERLI All decedents proxy vs. none of living sample. Living sample 1 year later than decedents

TAGER2 Age distribution for both men and women in study sample was significantly different from general population from which sample was drawn. Subjects who smoked but did not inhale were excluded

TVERDA Includes acute bronchitis

VOLLM1 Study population consists of volunteers who responded to extensive media advertising and cohort is biased towards those with respiratory disease, and analysis restricted to those with follow-up data. Subjects with abnormal baseline FEV were not invited to some phases so may have different follow-up rate

VOLLM2 Study population consists of volunteers who responded to extensive media advertising and cohort is biased towards those with respiratory disease, and analysis restricted to those with follow-up data. Subjects with abnormal baseline FEV were not invited to some phases so may have different follow-up rate WALD Includes ICD9: 416 (chronic pulmonary heart disease) and 519 (other diseases of respiratory system)

WIG Urban area is not a typical sample, as socio-economic status is above average

ZIETKO Small clinical study, not clear how controls were selected

Note that weakness is in respect of the current review, and is not a criticism of the original study which may have been designed with different objectives.

${ }^{9}$ Study conducted in employed or occupational group:

DOPICO outdoor workers for city and power company

FLETCH men-postmen, women-clerical workers

JOSHI employees at machine tool factory and woollen hosiery mill

KLAYTO employees at two research facilities

LAM1 employees in a machine factory

SHARP clerical and light assembly workers at power company

SUADIC armed forces, customs service, railway, telephone, post, banking and construction companies

WAGEN2 heterogeneous population of employees from different companies and organisations

WOOLF employees of large commercial firms

h Study conducted in mixed groups:

HAENSZ nationwide sample plus siblings of migrants to USA still resident in Norway

HAWTHO occupational groups (from industry, not otherwise specified) and census-identified sample

KATANC whites from Medicare, blacks from general population 
(58, 30.1\%), but less so by age of starting to smoke (17, $8.8 \%)$, duration of smoking $(12,6.2 \%)$ or duration of quitting $(18,9.3 \%)$.

Of the 116 principal studies of COPD, outcome is based on ICD codes in 29 (25.0\%), and lung function only in 59 (50.9\%). The GOLD criteria are used in 27 (23.2\%) studies, with MRC, ATS, ERS or BTS criteria used in 12 (10.3\%). In 69 (59.5\%) studies the subjects' asthma status is ignored, in $18(15.5 \%)$ all asthmatics subjects are excluded, and in $14(12.1 \%)$ the disease definition includes asthma. Only $19(16.4 \%)$ of the 116 principal studies mention conducting spirometry after use of a bronchodilator. The outcome is based on prevalence in $79(68.1 \%)$ principal studies, mortality in $28(24.1 \%)$ and incidence in 10 (8.6\%). In the principal studies, the median number of subjects is 2,033, and of cases 131 (range 13 to 32,822 ).

Of the 87 principal studies of $\mathrm{CB}$, the outcome is based on symptoms (not lung function) in 59 (67.8\%), and on ICD in only six (6.9\%). Other studies use selfreport, a doctor diagnosis, or other definitions. The MRC questionnaire is used in 21 (24.1\%). Asthmatics are excluded totally from six (6.9\%) studies, with asthmatics excluded only from the controls in three (3.4\%). The outcome is based on prevalence in 78 (89.7\%) of the principal studies, mortality in six (6.9\%) and incidence in three (3.4\%). The median number of subjects is 2,826, and of cases 193.5 (range 2 to 4,769).

Of the 26 principal studies of emphysema, the outcome is based on visual comparison in 10 (38.5\%), on diagnosis in seven $(26.9 \%)$, on ICD codes in five (19.2\%) and on other sources including self-report in four (15.4\%). Asthmatics are excluded in two (7.7\%) studies. The outcome is based on prevalence in 19 (73.1\%) of the studies, on mortality in five (19.2\%) and on incidence in two $(7.7 \%)$. The median number of subjects is 2,433, and of cases 96.5 (range 2 to 1384).

\section{Relative risks}

A total of 3,538 RRs are entered, 1,578 for COPD, 1,689 for $\mathrm{CB}$ and 271 for emphysema, the number recorded per study varying from 1 to 211 . Some 675 relate to subsidiary studies. Table 4 summarizes the distribution of various characteristics of the RRs by outcome, by study type for the principal studies, and overall. For fuller distributions of the RRs, referred to as necessary below, see Additional file 3.

Of the 2,863 RRs in principal studies, $67.8 \%$ relate to cross-sectional, $19.8 \%$ to prospective, and $12.4 \%$ to casecontrol studies. $81.2 \%$ of RRs are sex-specific. About half the RRs (52.0\%) are adjusted for one or more variables. Of 1,488 adjusted RRs, age is adjusted for in 1,382 (92.9\%) but only 490 (32.9\%) are adjusted for variables other than age, sex or other smoking aspects. $34.0 \%$ of the RRs are given directly or calculated from a $2 \times 2$ or $2 \times 2 \times \ell$ table, the rest being derived.

Of the 3,538 RRs, 1,439 are for major smoking indices, and 2,099 for dose-related indices (including 236 and 439 respectively in subsidiary studies). Of the 1,203 RRs in principal studies for major indices, $34.6 \%$ are for ever smoking, $37.8 \%$ current smoking and $27.6 \%$ ex smoking. $53.6 \%$ are for cigarette smoking ignoring other products, $33.8 \%$ any product smoking, and $12.6 \%$ cigarettes only. The unexposed group is typically never any product (55.8\%) or never cigarettes (43.1\%).

The distribution of smoking status for the 1,660 RRs in principal studies for dose-related indices differs considerably, with $22.8 \%$ for ever smoking, $59.6 \%$ current smoking and $17.6 \%$ ex smoking. Again, most (59.8\%) RRs relate to cigarette smoking ignoring other products. The unexposed group is never smoking (any product or cigarettes) for $50.4 \%$ of these RRs, low smoking for $39.2 \%$, and current smoking for $3.9 \%$. 52.7\% of RRs are for amount smoked, $8.1 \%$ age of starting, $19.8 \%$ packyears, $4.4 \%$ years duration, and $15.1 \%$ years quit (about half compared to never smokers or long-term quitters, the rest compared to current smokers or short-term quitters). Based on RRs with an unexposed base of never smoking, there are 174 sets of categorical data for amount smoked, 18 for age of starting, 52 for packyears, 11 for duration of smoking, and 26 for duration of quitting. For emphysema, there are few dose-related data other than for amount smoked

None of the RRs included in the meta-analyses and meta-regressions show more than minor failures of the validation tests used, attributable to rounding errors or small imprecisions or uncertainties in estimating the RRs and CIs. Additional File 3 provides further detail. 
Table 3 Distribution of the main characteristics of the 218 studies of COPD, CB and/or emphysema

\begin{tabular}{|c|c|c|c|c|c|c|c|c|c|}
\hline \multirow[b]{2}{*}{ Characteristic } & \multirow[b]{2}{*}{ Level } & \multicolumn{3}{|c|}{ Disease studied (principal studies) } & \multicolumn{3}{|c|}{ Study type (principal studies) ${ }^{a}$} & \multirow{2}{*}{$\begin{array}{r}\text { Subsidiary } \\
\text { studies }\end{array}$} & \multirow{2}{*}{$\begin{array}{r}\text { Al } \\
\text { studies }\end{array}$} \\
\hline & & COPD & $\mathrm{CB}$ & EMP & $\mathrm{CC}$ & Prosp & Cross-sec & & \\
\hline \multirow[t]{2}{*}{ Study status } & Principal & 116 & 87 & 26 & 20 & 39 & 134 & - & 193 \\
\hline & Subsidiary & (17) & $(14)$ & (2) & - & - & - & 25 & 25 \\
\hline \multirow[t]{4}{*}{ Study type } & Case/control & 14 & 7 & 0 & 20 & - & - & 2 & 22 \\
\hline & Prospective & 35 & 9 & 7 & - & 39 & - & 8 & 47 \\
\hline & Cross-sectional & 67 & 71 & 19 & - & - & 134 & 14 & 148 \\
\hline & Nested case/control & 0 & 0 & 0 & - & - & - & 1 & 1 \\
\hline \multirow[t]{3}{*}{ Study sex } & Both & 89 & 62 & 18 & 17 & 20 & 108 & 20 & 165 \\
\hline & Male & 23 & 20 & 8 & 2 & 17 & 23 & 5 & 47 \\
\hline & Female & 4 & 5 & 0 & 1 & 2 & 3 & 0 & 6 \\
\hline \multirow[t]{5}{*}{ Lowest age $^{\mathrm{b}}$} & $<20$ & 26 & 22 & 10 & 5 & 4 & 42 & 3 & 54 \\
\hline & $20-29$ & 28 & 25 & 2 & 3 & 10 & 32 & 9 & 54 \\
\hline & $30-39$ & 23 & 12 & 4 & 6 & 15 & 11 & 6 & 38 \\
\hline & $40-49$ & 26 & 17 & 4 & 5 & 7 & 29 & 5 & 46 \\
\hline & $50+$ & 13 & 11 & 6 & 1 & 3 & 20 & 2 & 26 \\
\hline \multirow[t]{6}{*}{ Highest age ${ }^{b}$} & $<50$ & 3 & 6 & 0 & 0 & 2 & 6 & 1 & 9 \\
\hline & $50-59$ & 4 & 8 & 0 & 0 & 4 & 8 & 1 & 13 \\
\hline & $60-69$ & 20 & 21 & 6 & 2 & 9 & 29 & 5 & 45 \\
\hline & $70-79$ & 19 & 12 & 1 & 4 & 6 & 19 & 8 & 37 \\
\hline & $80+$ & 70 & 39 & 19 & 14 & 18 & 71 & 10 & 113 \\
\hline & Unknown & 0 & 1 & 0 & 0 & 0 & 1 & 0 & 1 \\
\hline \multirow[t]{9}{*}{ Region } & USA/Canada & 31 & 28 & 12 & 2 & 14 & 40 & 7 & 63 \\
\hline & S/C America & 7 & 2 & 0 & 2 & 0 & 6 & 0 & 8 \\
\hline & UK & 11 & 13 & 1 & 5 & 6 & 12 & 5 & 28 \\
\hline & Western Europe & 8 & 9 & 1 & 4 & 2 & 11 & 1 & 18 \\
\hline & Scandinavia & 18 & 16 & 5 & 0 & 6 & 26 & 7 & 39 \\
\hline & Other Europe & 12 & 4 & 0 & 2 & 1 & 11 & 4 & 18 \\
\hline & Asia & 22 & 10 & 6 & 4 & 7 & 21 & 1 & 33 \\
\hline & Other & 3 & 4 & 1 & 1 & 1 & 5 & 0 & 7 \\
\hline & Multicountry & 4 & 1 & 0 & 0 & 2 & 2 & 0 & 4 \\
\hline \multirow[t]{7}{*}{ Start year of study } & $<1960$ & 7 & 10 & 3 & 2 & 7 & 4 & 3 & 16 \\
\hline & 1960-1969 & 19 & 15 & 4 & 1 & 12 & 17 & 8 & 38 \\
\hline & 1970-79 & 15 & 19 & 5 & 1 & 9 & 23 & 6 & 39 \\
\hline & 1980-89 & 12 & 10 & 4 & 2 & 5 & 13 & 2 & 22 \\
\hline & 1990-99 & 23 & 16 & 6 & 2 & 6 & 29 & 3 & 40 \\
\hline & $>1999$ & 25 & 1 & 0 & 2 & 0 & 24 & 1 & 27 \\
\hline & Unknown & 15 & 16 & 4 & 10 & 0 & 24 & 2 & 36 \\
\hline Major study weakness & Present & 19 & 5 & 2 & 9 & 4 & 13 & 6 & 32 \\
\hline \multirow[t]{8}{*}{ Smoking results available } & Current $v$ never & 89 & 70 & 15 & 14 & 34 & 98 & 23 & 169 \\
\hline & Ex $\vee$ never & 82 & 65 & 12 & 12 & 29 & 93 & 19 & 153 \\
\hline & Ever $\vee$ never & 95 & 71 & 20 & 16 & 29 & 113 & 18 & 176 \\
\hline & Amount smoked & 39 & 42 & 10 & 7 & 23 & 47 & 14 & 91 \\
\hline & Age started & 13 & 3 & 1 & 5 & 7 & 5 & 3 & 20 \\
\hline & Pack years & 42 & 13 & 3 & 10 & 5 & 43 & 5 & 63 \\
\hline & Duration of smoking & 6 & 4 & 3 & 2 & 4 & 6 & 2 & 14 \\
\hline & Duration of quitting & 8 & 7 & 2 & 3 & 8 & 7 & 4 & 22 \\
\hline \multirow[t]{5}{*}{ Outcomes } & COPD only & 93 & - & - & 13 & 28 & 52 & 11 & 104 \\
\hline & CB only & - & 63 & - & 6 & 2 & 55 & 7 & 70 \\
\hline & Emp only & - & - & 9 & 0 & 1 & 8 & 0 & 9 \\
\hline & More than one & 23 & 24 & 17 & 1 & 8 & 19 & 7 & 35 \\
\hline & COPD & 116 & - & - & 14 & 35 & 67 & 17 & 133 \\
\hline
\end{tabular}


Table 3 Distribution of the main characteristics of the 218 studies of COPD, CB and/or emphysema (Continued)

\begin{tabular}{llrrrrrrrr}
\hline & CB & - & 87 & - & 7 & 9 & 71 & 14 & 101 \\
& Emp & - & - & 26 & 0 & 7 & 19 & 2 \\
All studies & & 116 & 87 & 26 & 20 & 39 & 134 & 25 & 218 \\
& & & & & & & & & \\
\end{tabular}

${ }^{a} \mathrm{CC}=$ case control, Prosp $=$ prospective, Cross-sec $=$ cross-sectional

${ }^{\mathrm{b}}$ At start of study.

Table 4 Distribution of the main characteristics of the relative risks (principal studies only ${ }^{a}$ )

\begin{tabular}{|c|c|c|c|c|c|c|}
\hline \multirow[t]{2}{*}{ RRs } & \multirow[t]{2}{*}{ Characteristic } & \multirow[t]{2}{*}{ Level } & \multicolumn{4}{|c|}{ Outcome } \\
\hline & & & COPD & CB & Emphysema & Total \\
\hline \multirow[t]{15}{*}{ All RRs } & Total & Total & 1,342 & 1,311 & 210 & 2,863 \\
\hline & Study type & Case/control & 194 & 162 & 0 & 356 \\
\hline & & Prospective & 414 & 86 & 66 & 566 \\
\hline & & Cross-sectional & 734 & 1,063 & 144 & 1,941 \\
\hline & Sex & Both & 289 & 215 & 35 & 539 \\
\hline & & Male & 660 & 552 & 114 & 1,326 \\
\hline & & Female & 393 & 544 & 61 & 998 \\
\hline & Adjusted for any variable & No & 605 & 677 & 93 & 1,375 \\
\hline & & Yes & 737 & 634 & 117 & 1,488 \\
\hline & Adjusted for age & No & 624 & 758 & 99 & 1,481 \\
\hline & & Yes & 718 & 553 & 111 & 1,382 \\
\hline & Adjusted for other confounders ${ }^{b}$ & No & 1,051 & 1,135 & 187 & 2,373 \\
\hline & & Yes & 291 & 176 & 23 & 490 \\
\hline & Derivation & Original or $2 \times 2^{c}$ & 409 & 478 & 86 & 973 \\
\hline & & Other derived & 933 & 833 & 124 & 1890 \\
\hline \multirow[t]{10}{*}{ RRs for major smoking indices } & Total & Total & 551 & 547 & 105 & 1,203 \\
\hline & Exposed group: smoking status & Ever & 185 & 186 & 45 & 416 \\
\hline & & Current & 214 & 205 & 36 & 455 \\
\hline & & Ex & 152 & 156 & 24 & 332 \\
\hline & Exposed group: smoking product & Any product & 166 & 211 & 29 & 406 \\
\hline & & Cigarettes (+/- other) & 314 & 269 & 62 & 645 \\
\hline & & Cigarettes only & 71 & 67 & 14 & 152 \\
\hline & Unexposed & Never any product & 283 & 335 & 53 & 671 \\
\hline & & Never cigarettes & 261 & 206 & 52 & 519 \\
\hline & & Other $^{d}$ & 7 & 6 & 0 & 13 \\
\hline \multirow[t]{11}{*}{ RRs for all dose-response indices } & Total & Total & 791 & 764 & 105 & 1,660 \\
\hline & Exposed group: smoking status & Ever & 221 & 138 & 19 & 378 \\
\hline & & Current & 415 & 492 & 83 & 990 \\
\hline & & Ex & 155 & 134 & 3 & 292 \\
\hline & Exposed group: smoking product & Any product & 64 & 176 & 8 & 248 \\
\hline & & Cigarettes (+/- other) & 525 & 399 & 69 & 993 \\
\hline & & Cigarettes only & 202 & 189 & 28 & 419 \\
\hline & Unexposed & Never (any or cigs) & 389 & 387 & 60 & 836 \\
\hline & & Low (any or cigs) & 318 & 295 & 38 & 651 \\
\hline & & Current (any or cigs) & 33 & 32 & 0 & 65 \\
\hline & & Other ${ }^{\mathrm{f}} /$ not applicable $\mathrm{e}^{\mathrm{g}}$ & 51 & 50 & 7 & 108 \\
\hline \multirow[t]{4}{*}{ RRs for amount smoked } & Total & Total & 335 & 474 & 65 & 874 \\
\hline & Exposed group: smoking status & Ever & 3 & 51 & 9 & 63 \\
\hline & & Current & 323 & 413 & 55 & 791 \\
\hline & & Ex & 9 & 10 & 1 & 20 \\
\hline Sets for amount smoked & & vs. never (any or cigs) & 66 & 94 & 14 & 174 \\
\hline
\end{tabular}


Table 4 Distribution of the main characteristics of the relative risks (principal studies only ${ }^{\mathrm{a}}$ ) (Continued)

\begin{tabular}{|c|c|c|c|c|c|c|}
\hline & & vs. low amount & 65 & 97 & 16 & 178 \\
\hline & & vs. non ${ }^{h}$ & 2 & 9 & 0 & 11 \\
\hline & & Non categorical & 9 & 11 & 3 & 23 \\
\hline \multirow[t]{4}{*}{ RRs for age started } & Total & Total & 78 & 50 & 6 & 134 \\
\hline & Exposed group: smoking status & Ever & 6 & 38 & 0 & 44 \\
\hline & & Current & 65 & 12 & 6 & 83 \\
\hline & & Ex & 7 & 0 & 0 & 7 \\
\hline \multirow[t]{4}{*}{ Sets for age started } & & vs. never (any or cigs) & 12 & 4 & 2 & 18 \\
\hline & & vs. later start & 21 & 9 & 2 & 32 \\
\hline & & vs. non ${ }^{h}$ & 1 & 0 & 0 & 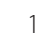 \\
\hline & & Non categorical & 6 & 0 & 0 & 6 \\
\hline \multirow[t]{4}{*}{ RRs for pack-years } & Total & Total & 225 & 88 & 15 & 328 \\
\hline & Exposed group: smoking status & Ever & 208 & 48 & 5 & 261 \\
\hline & & Current & 14 & 30 & 10 & 54 \\
\hline & & Ex & 3 & 10 & 0 & 13 \\
\hline \multirow[t]{3}{*}{ Sets for pack-years } & & vs. never (any or cigs) & 34 & 15 & 3 & 52 \\
\hline & & vs. low pack-years & 41 & 15 & 3 & 59 \\
\hline & & Non categorical & 25 & 9 & 2 & 36 \\
\hline \multirow[t]{4}{*}{ RRs for duration of smoking } & Total & Total & 17 & 39 & 17 & 73 \\
\hline & Exposed group: smoking status & Ever & 4 & 1 & 5 & 10 \\
\hline & & Current & 13 & 37 & 12 & 62 \\
\hline & & Ex & 0 & 1 & 0 & \\
\hline \multirow[t]{3}{*}{ Sets for duration of smoking } & & vs. never (any or cigs) & 2 & 6 & 3 & 11 \\
\hline & & vs. low duration & 2 & 6 & 2 & 10 \\
\hline & & Non categorical & 5 & 5 & 0 & 10 \\
\hline RRs for duration of quitting & Total & Total & 136 & 113 & 2 & 251 \\
\hline \multirow[t]{5}{*}{ Sets for duration of quitting } & & vs. never (any or cigs) & 13 & 13 & 0 & 26 \\
\hline & & vs. current & 12 & 11 & 0 & 23 \\
\hline & & vs. long term ex & 14 & 11 & 0 & 25 \\
\hline & & vs. recent ex & 14 & 11 & 0 & 25 \\
\hline & & Non categorical & 2 & 3 & 2 & \\
\hline
\end{tabular}

a The number of additional RRs recorded for subsidiary studies was 236 for COPD, 378 for CB and 61 for emphysema.

${ }^{\mathrm{b}}$ Variables other than sex, age and aspects of smoking

c Calculated directly from $2 \times 2$ or $2 \times 2 \times \ell$ table

${ }^{\mathrm{d}}$ Never or low amount (any or cigs)

${ }^{\mathrm{e}}$ Low in terms of the measure of exposure (e.g. low amount for amount smoked, later starting for age started)

${ }^{f}$ Never or low amount (any or cigs); or never and ex smokers combined

${ }^{g}$ Dose-response RR not for categorical data

${ }^{\mathrm{h}}$ Never and ex smokers combined

The meta-analyses and meta-regressions

The main findings are summarized in the following sections, with tables and forest plots. Fuller results of the meta-analyses for the major smoking variables are given in Additional file 4 for COPD, Additional file 5 for $C B$ and Additional file 6 for emphysema. Similar results for the dose-related smoking variables are given in Additional file 7 for COPD, Additional file 8 for CB and Additional file 9 for emphysema. An Excel file, available as Additional file 10 , allows the user readily to view selected results from all these meta-analyses. Detailed meta-regression outputs are given in Additional file 11. For dose-related indices,
Additional file 12 presents within-study plots of the doseresponse relationships, while Additional file 13 gives results that were originally presented in a form unsuitable for meta-analysis. The interested reader should first refer to Additional file 1, which describes the content and structure of all these Additional files.

\section{A. Risk from ever smoking}

Figures 1 and 2 (COPD), 3 and 4 (CB) and 5 (emphysema) present the results of the main meta-analyses for ever smoking any product (or cigarette smoking from studies without RRs for any product), based on mostadjusted RRs. Additional results subdivided by level of 


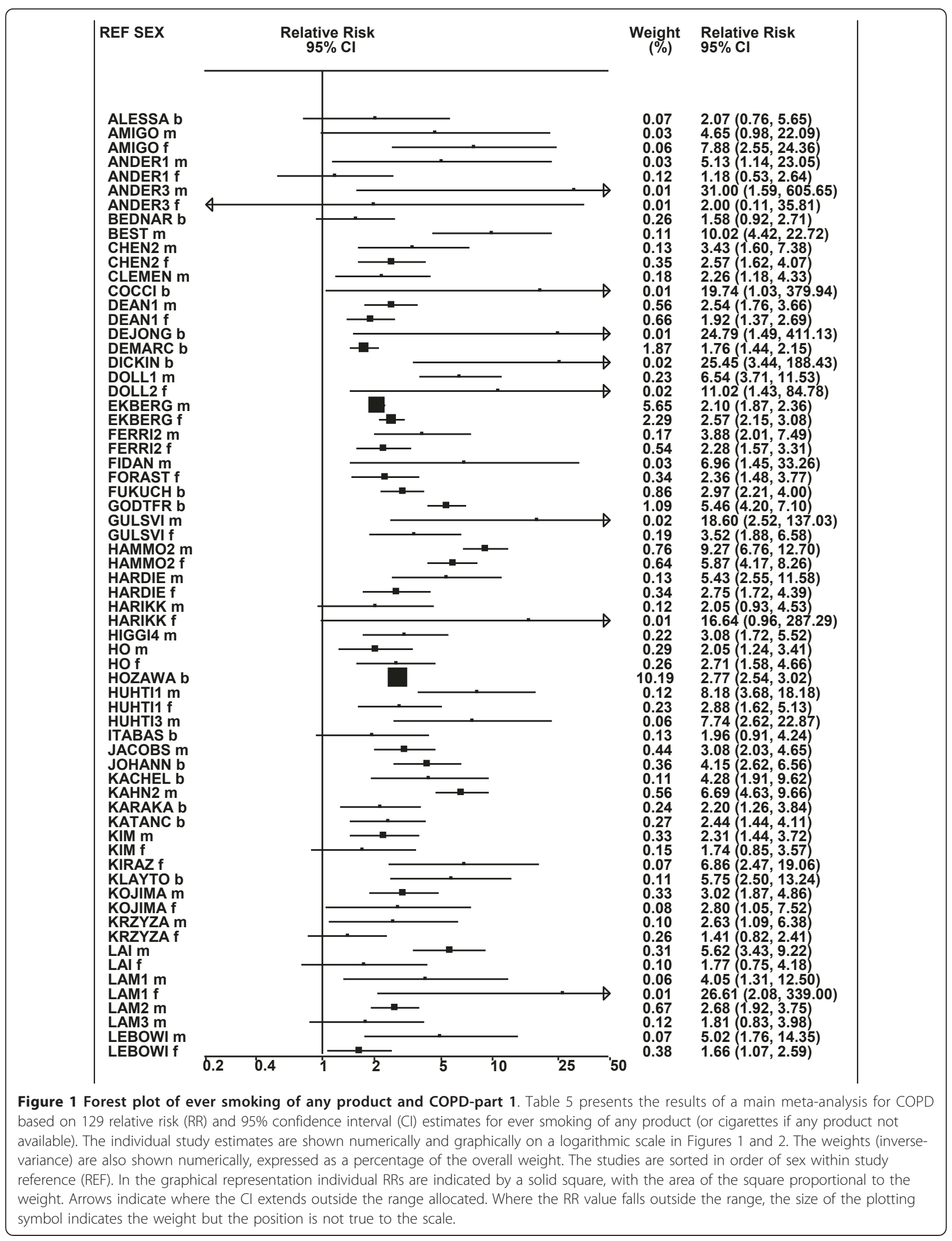




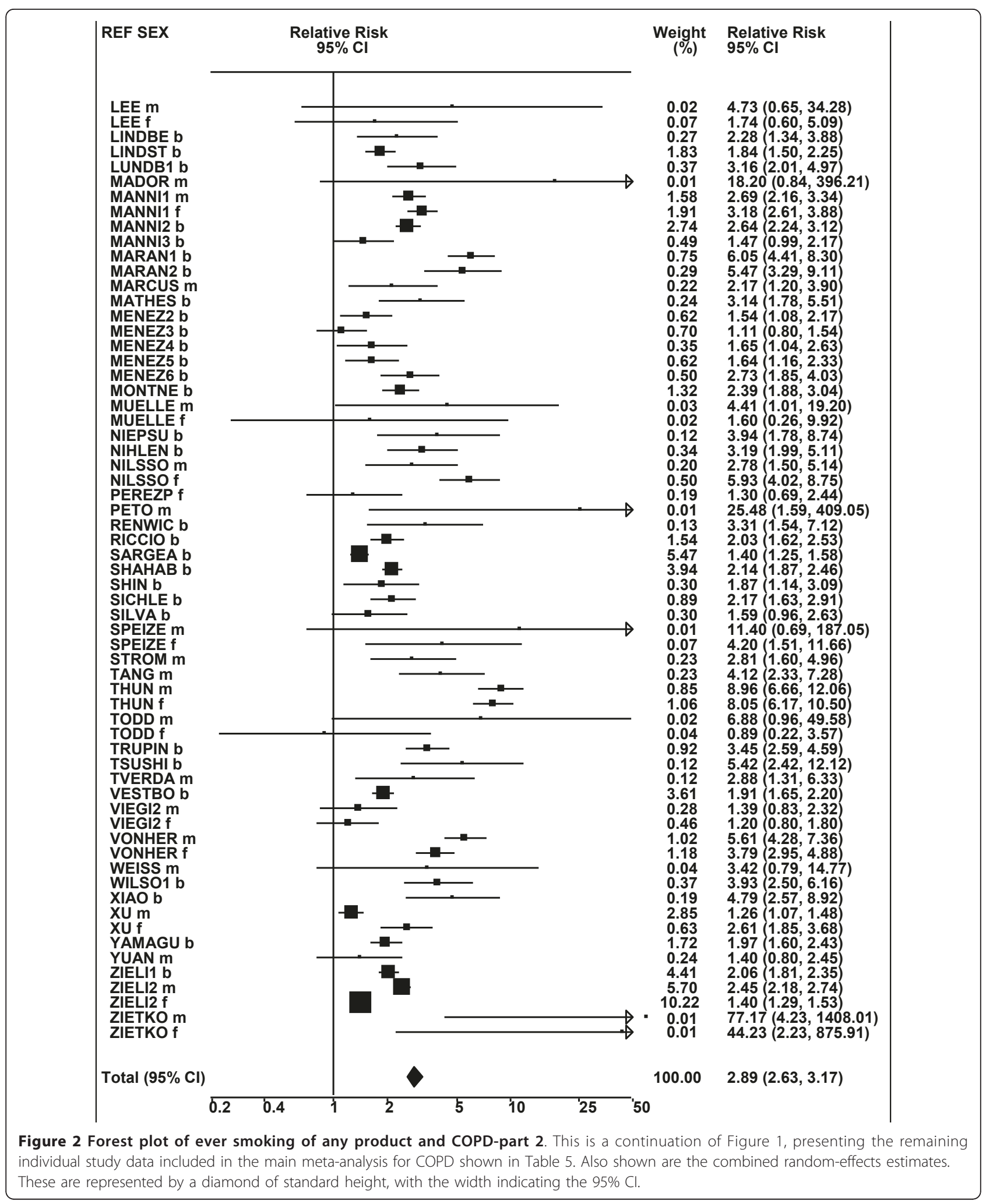




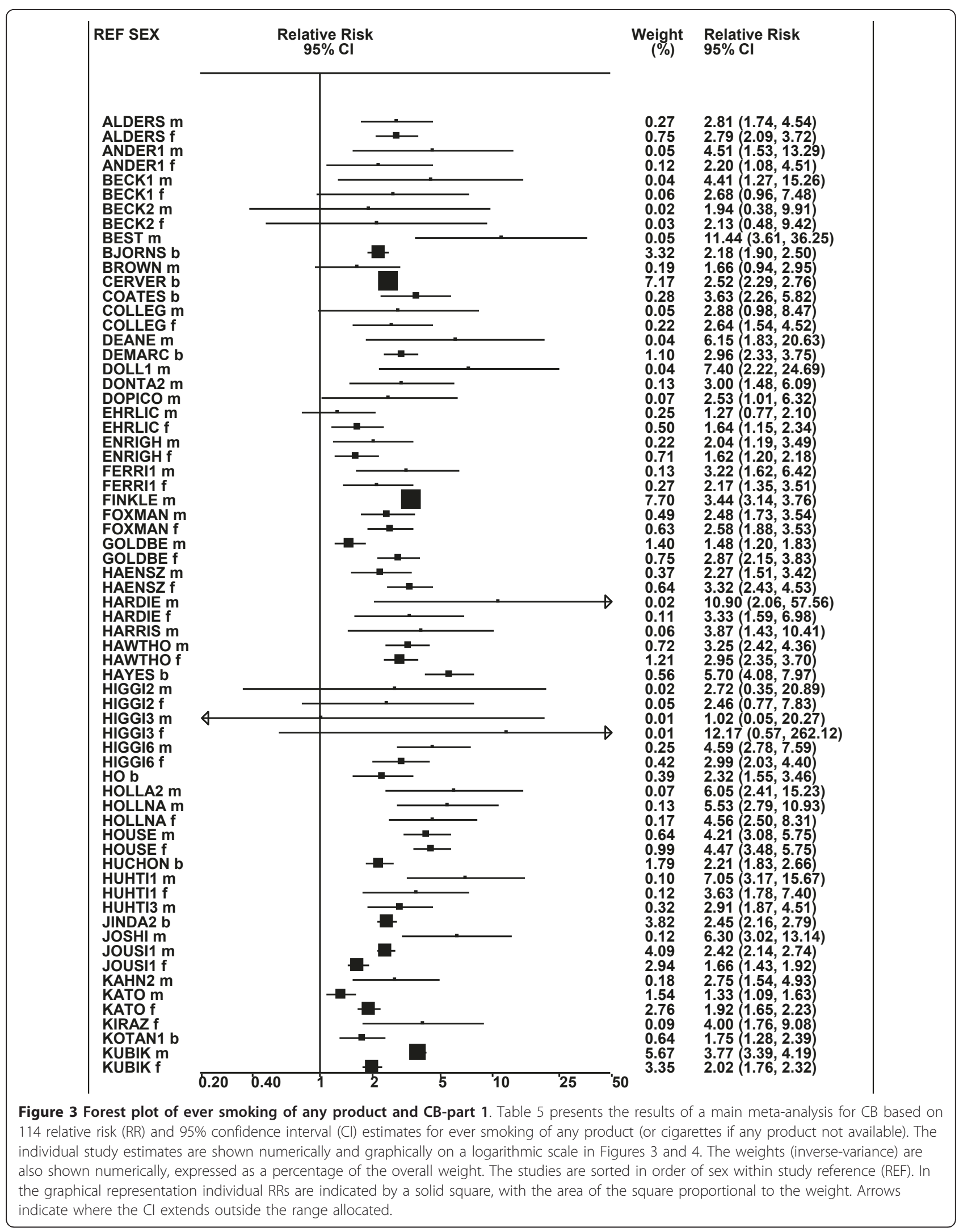




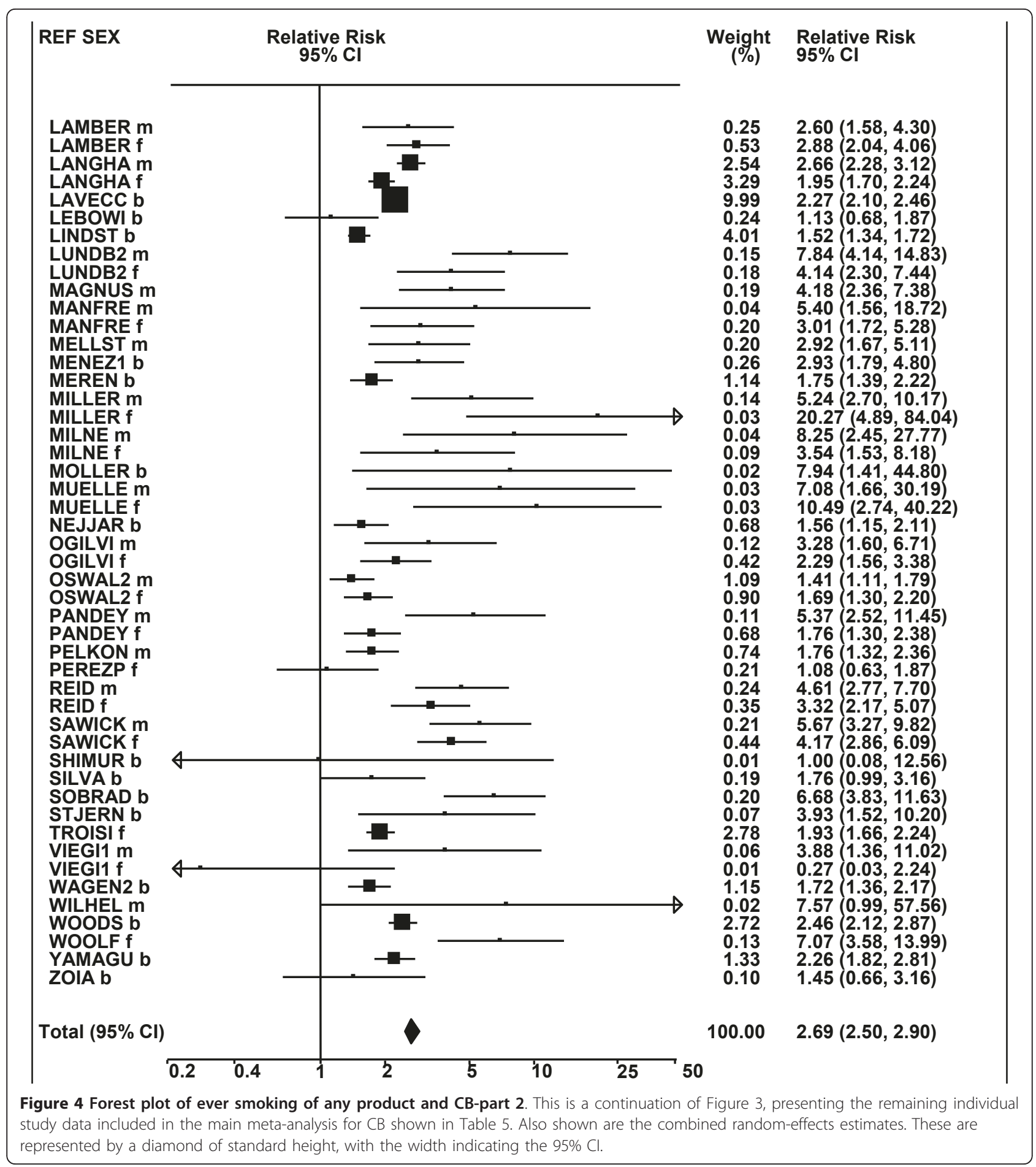

certain characteristics are shown in Table 5. From these findings, various observations can be made.

First, the RRs for all three outcomes are markedly heterogeneous, with $\mathrm{H}$ at least 5 for all three diseases $(\mathrm{p}<$ 0.001). Individual RRs vary up to 77.17 for COPD (study ZIETKO for males), 20.27 for CB (MILLER/females) and
16.58 for emphysema (HUHTI/males). Based on randomeffects estimates, a positive association is seen, strongest for emphysema (RR 4.51, 95\% CI 3.38-6.02, based on 28 RRs), but also clearly evident for COPD $(2.89,2.63-3.17, \mathrm{n}$ $=129)$ and $C B(2.69,2.50-2.90, \mathrm{n}=114)$. Although the strength of association varies markedly by study, the 


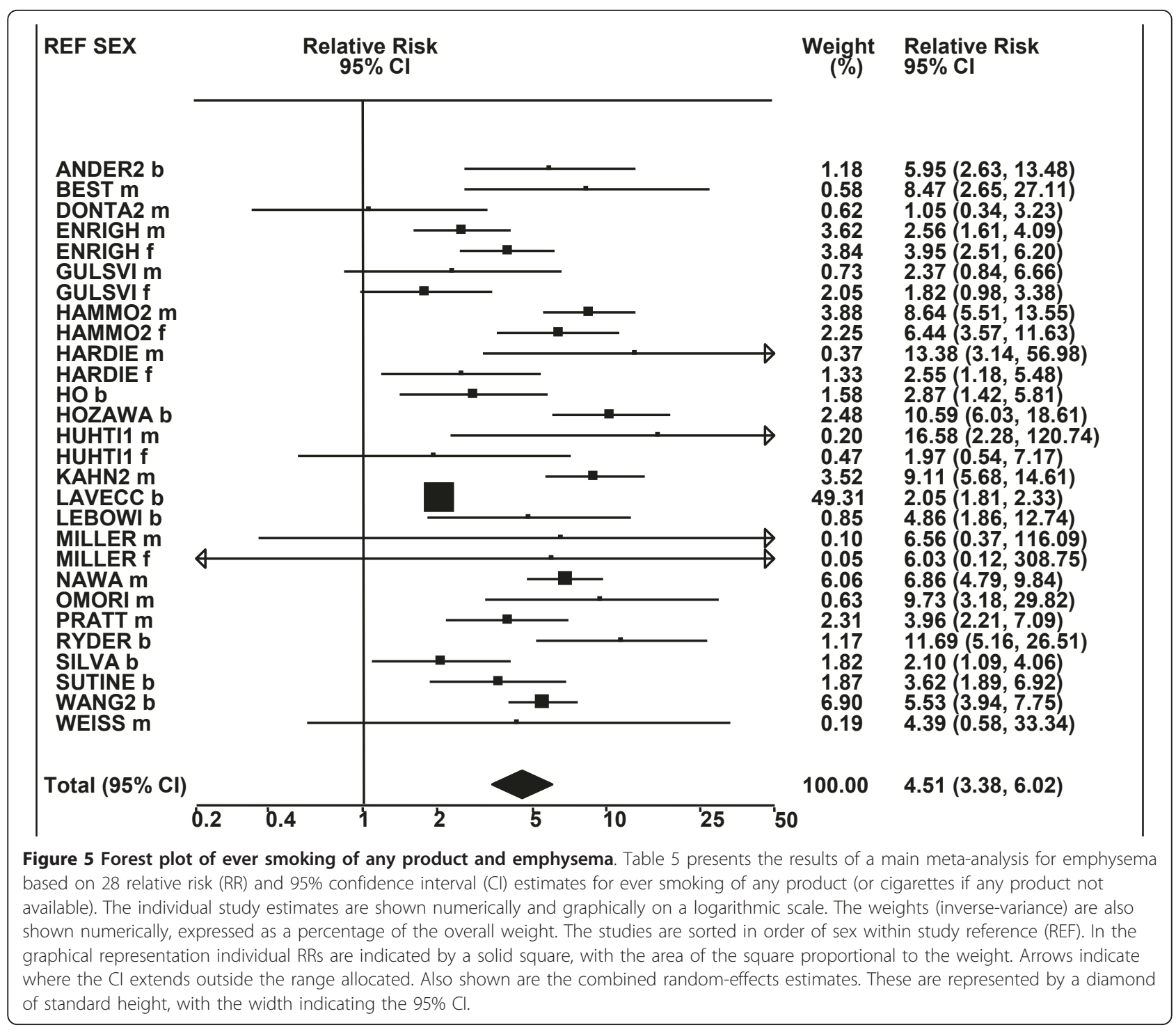

consistency of direction is clear, with only one of the 129 COPD RRs, one of the 114 CB RRs, and none of the 28 emphysema RRs below 1.0.

These estimates are little affected by preferring RRs for ever smoking cigarettes to those for ever smoking any product, the random-effects estimates changing to $2.92(2.65-3.20)$ for COPD, 2.70 (2.50-2.91) for CB and 4.57 (3.40-6.15) for emphysema. This is partly due to many studies providing only one type of RR, so that for COPD, for example, 117 of the 129 RRs are common to both meta-analyses. Nor are they affected by preferring least-adjusted, rather than most-adjusted RRs, with the estimates now $2.85(2.59-3.15, \mathrm{n}=133)$ for COPD, 2.73 $(2.52-2.95, \mathrm{n}=119)$ for $\mathrm{CB}$ and $4.16(3.03-5.71, \mathrm{n}=30)$ for emphysema, slightly more RRs being included as some studies have sex-specific unadjusted RRs but only sexes combined adjusted RRs.
Returning to the main meta-analysis (most-adjusted and preferring ever smoking any product), there is also large variation between RRs in the weight they contribute to the analysis. For COPD, of a total weight of 5,116 for the 129 RRs (mean 39.7), the largest weight is 523 for study ZIELI2 for females, with six other RRs having weights of over 200. For CB, of the total of 6,146 for the 114 RRs (mean 53.9), the largest weight is 614 for study LAVECC for sexes combined, with eight other RRs having weights over 200. For emphysema, where the total weight is much lower, 489 (mean 17.5 for the 28 RRs), the weight of 241 for LAVECC for the sexes combined RR contributes almost a half.

In investigating sources of heterogeneity, variation was studied firstly using a univariate approach, the results for the characteristics considered in Table 5 being summarized below. 
Table 5 Main meta-analyses for ever smoking of any product (or cigarettes if any product not available) ${ }^{a}$

\begin{tabular}{|c|c|c|c|c|c|}
\hline Characteristic & Level & Statistic $^{\mathrm{b}}$ & COPD & $\mathrm{CB}$ & Emphysema \\
\hline \multirow[t]{4}{*}{ All } & All & $n$ & 129 & 114 & 28 \\
\hline & & $\mathrm{F}$ & $2.34(2.27-2.40)$ & $2.42(2.36-2.48)$ & $3.22(2.95-3.52)$ \\
\hline & & $\mathrm{R}$ & $2.89(2.63-3.17)$ & $2.69(2.50-2.90)$ & $4.51(3.38-6.02)$ \\
\hline & & $\mathrm{H}, \mathrm{P}_{\mathrm{H}}$ & $8.11,<0.001$ & $5.81,<0.001$ & $6.42,<0.001$ \\
\hline \multirow[t]{10}{*}{ Sex } & Male & $n$ & 49 & 51 & 13 \\
\hline & & $\mathrm{F}$ & $2.69(2.55-2.84)$ & $2.87(2.74-3.00)$ & $5.71(4.74-6.87)$ \\
\hline & & $\mathrm{R}$ & $3.60(2.98-4.34)$ & $3.18(2.77-3.65)$ & $5.42(3.69-7.96)$ \\
\hline & Female & $n$ & 35 & 39 & 6 \\
\hline & & $\mathrm{F}$ & $2.16(2.04-2.28)$ & $2.22(2.11-2.32)$ & $3.44(2.60-4.55)$ \\
\hline & & $\mathrm{R}$ & $2.73(2.17-3.43)$ & $2.57(2.28-2.89)$ & $3.25(2.07-5.10)$ \\
\hline & Combined & $\mathrm{n}$ & 45 & 24 & 9 \\
\hline & & $\mathrm{F}$ & $2.26(2.17-2.35)$ & $2.24(2.15-2.33)$ & $2.62(2.36-2.92)$ \\
\hline & & $\mathrm{R}$ & $2.51(2.23-2.82)$ & $2.29(2.05-2.57)$ & $4.47(2.72-7.34)$ \\
\hline & Between levels & $P_{B}$ & NS & $<0.001$ & $<0.05$ \\
\hline \multirow[t]{13}{*}{ Continent } & N America & $n$ & 35 & 38 & 14 \\
\hline & & $F$ & 3.21 (3.04-3.39) & $2.91(2.75-3.07)$ & $5.33(4.49-6.33)$ \\
\hline & & $\mathrm{R}$ & $3.48(2.88-4.20)$ & $3.17(2.70-3.71)$ & $5.32(3.86-7.32)$ \\
\hline & Europe & $n$ & 59 & 60 & 10 \\
\hline & & $\mathrm{F}$ & $2.09(2.01-2.17)$ & $2.34(2.27-2.41)$ & $2.19(1.95-2.46)$ \\
\hline & & $\mathrm{R}$ & $2.79(2.46-3.16)$ & $2.62(2.39-2.89)$ & $3.13(2.01-4.87)$ \\
\hline & Asia & $\mathrm{n}$ & 23 & 9 & 4 \\
\hline & & $\mathrm{F}$ & $2.26(2.08-2.45)$ & $2.08(1.92-2.24)$ & $5.76(4.59-7.23)$ \\
\hline & & $\mathrm{R}$ & $2.73(2.15-3.46)$ & $2.21(1.78-2.75)$ & 5.59 (3.93-7.95) \\
\hline & Other or multicountry & $\mathrm{n}$ & 12 & 7 & 0 \\
\hline & & $\mathrm{F}$ & $1.91(1.71-2.14)$ & $2.34(2.09-2.61)$ & \\
\hline & & $\mathrm{R}$ & $2.13(1.66-2.73)$ & $2.09(1.59-2.74)$ & \\
\hline & Between levels & $P_{B}$ & $<0.001$ & $<0.05$ & $<0.001$ \\
\hline \multirow[t]{13}{*}{ Publication year } & Before 1980 & $n$ & 25 & 49 & 13 \\
\hline & & $\mathrm{F}$ & $3.82(3.40-4.29)$ & $3.02(2.86-3.18)$ & $5.81(4.74-7.12)$ \\
\hline & & $\mathrm{R}$ & $3.79(2.82-5.10)$ & $3.22(2.82-3.68)$ & $5.37(3.72-7.75)$ \\
\hline & 1980-89 & $\mathrm{n}$ & 10 & 28 & 5 \\
\hline & & $\mathrm{F}$ & $2.13(1.80-2.50)$ & $2.44(2.33-2.56)$ & $2.10(1.86-2.37)$ \\
\hline & & $\mathrm{R}$ & $2.34(1.79-3.05)$ & $2.72(2.33-3.17)$ & $2.39(1.49-3.82)$ \\
\hline & 1990-99 & $\mathrm{n}$ & 19 & 17 & 3 \\
\hline & & $\mathrm{F}$ & $3.75(3.36-4.18)$ & $2.09(1.96-2.22)$ & $3.14(2.34-4.22)$ \\
\hline & & $\mathrm{R}$ & $3.31(2.39-4.58)$ & $2.24(1.91-2.63)$ & $3.14(2.34-4.22)$ \\
\hline & 2000 or later & $n$ & 75 & 20 & 7 \\
\hline & & $\mathrm{F}$ & $2.19(2.13-2.26)$ & $2.19(2.09-2.29)$ & $5.76(4.72-7.04)$ \\
\hline & & $\mathrm{R}$ & $2.62(2.36-2.90)$ & $2.19(1.93-2.48)$ & $5.58(3.63-5.58)$ \\
\hline & Between levels & $P_{B}$ & $<0.001$ & $<0.001$ & $<0.001$ \\
\hline \multirow[t]{10}{*}{ Study type } & Case-control & $\mathrm{n}$ & 16 & 10 & 0 \\
\hline & & $\mathrm{F}$ & $2.51(2.10-2.99)$ & $2.35(2.04-2.69)$ & \\
\hline & & $\mathrm{R}$ & $2.95(2.14-4.06)$ & $2.72(1.85-3.77)$ & \\
\hline & Prospective & $\mathrm{n}$ & 37 & 11 & 6 \\
\hline & & $\mathrm{F}$ & $4.49(4.12-4.89)$ & $2.30(2.08-2.55)$ & $6.12(4.77-7.85)$ \\
\hline & & $\mathrm{R}$ & $3.71(2.94-4.69)$ & $2.60(2.04-3.30)$ & $4.95(2.72-8.99)$ \\
\hline & Cross-sectional & $\mathrm{n}$ & 76 & 93 & 22 \\
\hline & & $\mathrm{F}$ & $2.16(2.10-2.22)$ & $2.43(2.36-2.49)$ & $2.93(2.67-3.22)$ \\
\hline & & $\mathrm{R}$ & $2.54(2.32-2.80)$ & $2.70(2.49-2.93)$ & $4.36(3.19-5.96)$ \\
\hline & Between levels & $P_{B}$ & $<0.001$ & NS & $<0.05$ \\
\hline Outcome subtype & Mortality & $\mathrm{n}$ & 29 & 3 & 4 \\
\hline
\end{tabular}


Table 5 Main meta-analyses for ever smoking of any product (or cigarettes if any product not available) ${ }^{\text {a }}$ (Continued)

\begin{tabular}{|c|c|c|c|c|c|}
\hline & & $\mathrm{F}$ & $4.50(4.09-4.94)$ & $4.10(2.54-6.60)$ & $8.24(6.24-10.87)$ \\
\hline & & $\mathrm{R}$ & $3.95(3.00-5.21)$ & $5.49(2.14-14.11)$ & $8.24(6.24-10.87)$ \\
\hline & $\begin{array}{l}\text { Lung function (COPD) or } \\
\text { symptoms (CB) }\end{array}$ & $\mathrm{n}$ & 60 & 83 & 0 \\
\hline & & $\mathrm{F}$ & $2.16(2.09-2.23)$ & $2.51(2.44-2.58)$ & \\
\hline & & $\mathrm{R}$ & $2.35(2.13-2.59)$ & $2.78(2.55-3.03)$ & \\
\hline & Other & $\mathrm{n}$ & 40 & 28 & 24 \\
\hline & & $\mathrm{F}$ & $2.31(2.19-2.45)$ & $2.11(2.00-2.22)$ & $2.89(2.63-3.18)$ \\
\hline & & $\mathrm{R}$ & $3.10(2.56-3.76)$ & $2.31(2.01-2.65)$ & $4.00(2.97-5.37)$ \\
\hline & Between levels & $P_{B}$ & $<0.001$ & $<0.05$ & $<0.01$ \\
\hline \multirow[t]{13}{*}{ How asthma taken into account } & Ignored & $\mathrm{n}$ & 76 & 103 & 26 \\
\hline & & $\mathrm{F}$ & $2.60(2.52-2.70)$ & $2.44(2.38-2.50)$ & $3.22(2.94-3.52)$ \\
\hline & & $\mathrm{R}$ & $2.95(2.62-3.32)$ & $2.69(2.50-2.91)$ & $4.61(3.43-6.19)$ \\
\hline & Excluded & $\mathrm{n}$ & 21 & 7 & 2 \\
\hline & & $\mathrm{F}$ & $1.83(1.73-1.94)$ & $1.91(1.71-2.13)$ & $3.22(1.45-7.11)$ \\
\hline & & $\mathrm{R}$ & $2.35(1.92-2.87)$ & $2.13(1.61-2.80)$ & $3.20(0.36-28.37)$ \\
\hline & Included in outcome definition & $\mathrm{n}$ & 19 & - & - \\
\hline & & $\mathrm{F}$ & $1.76(1.61-1.92)$ & & \\
\hline & & $\mathrm{R}$ & $2.55(2.00-3.26)$ & & \\
\hline & Other & $\mathrm{n}$ & 13 & 4 & 0 \\
\hline & & $\mathrm{F}$ & $3.68(3.20-4.24)$ & $5.41(3.58-8.18)$ & \\
\hline & & $\mathrm{R}$ & $3.62(2.80-4.69)$ & $5.37(3.13-9.21)$ & \\
\hline & Between levels & $P_{B}$ & $<0.001$ & $<0.1$ & NS \\
\hline \multirow[t]{13}{*}{ Number of cases } & $1-50$ & $\mathrm{n}$ & 23 & 17 & 6 \\
\hline & & $F$ & $4.06(3.12-5.29)$ & $2.70(1.98-3.69)$ & $2.89(1.78-4.67)$ \\
\hline & & $\mathrm{R}$ & $4.45(3.19-6.21)$ & $2.70(1.98-3.69)$ & $2.89(1.78-4.67)$ \\
\hline & $51-100$ & $\mathrm{n}$ & 29 & 13 & 9 \\
\hline & & $F$ & $2.42(2.12-2.75)$ & $3.71(2.91-4.72)$ & $2.87(2.15-3.83)$ \\
\hline & & $\mathrm{R}$ & $2.55(2.09-3.12)$ & $4.15(2.97-5.80)$ & $3.19(2.10-4.85)$ \\
\hline & $101-200$ & $\mathrm{n}$ & 30 & 19 & 5 \\
\hline & & $F$ & $2.39(2.18-2.63)$ & $3.07(2.66-3.53)$ & $7.30(5.31-10.03)$ \\
\hline & & $\mathrm{R}$ & $2.69(2.24-3.24)$ & $3.48(2.64-4.58)$ & $7.48(4.73-11.85)$ \\
\hline & $201+$ & $\mathrm{n}$ & 47 & 65 & 8 \\
\hline & & $\mathrm{F}$ & $2.31(2.24-2.38)$ & $2.38(2.32-2.44)$ & $3.03(2.74-3.24)$ \\
\hline & & $\mathrm{R}$ & $2.91(2.54-3.32)$ & $2.51(2.31-2.72)$ & $4.96(2.99-8.22)$ \\
\hline & Between levels & $P_{B}$ & NS & NS & NS \\
\hline \multirow[t]{7}{*}{ Analysis type } & Prevalence & $\mathrm{n}$ & 93 & 105 & 22 \\
\hline & & $F$ & $2.17(2.11-2.23)$ & $2.43(2.37-2.50)$ & $2.93(2.67-3.22)$ \\
\hline & & $\mathrm{R}$ & $2.57(2.35-2.81)$ & $2.71(2.51-2.93)$ & $4.36(3.19-5.96)$ \\
\hline & Onset & $n$ & 36 & 9 & 6 \\
\hline & & $F$ & $4.55(4.17-4.96)$ & $2.02(1.78-2.28)$ & $6.12(4.77-7.85)$ \\
\hline & & $\mathrm{R}$ & $3.77(2.98-4.78)$ & $2.37(1.80-3.13)$ & $4.95(2.72-8.99)$ \\
\hline & Between levels & $P_{B}$ & $<0.001$ & NS & $<0.05$ \\
\hline \multirow[t]{9}{*}{ Smoking product } & Any & $\mathrm{n}$ & 48 & 61 & 11 \\
\hline & & $\mathrm{F}$ & $2.60(2.47-2.74)$ & $2.59(2.51-2.68)$ & $2.51(2.24-2.80)$ \\
\hline & & $\mathrm{R}$ & $2.99(2.61-3.44)$ & $2.89(2.63-3.19)$ & $4.16(2.55-6.78)$ \\
\hline & $\begin{array}{l}\text { Cigarettes (ignoring other } \\
\text { products) }\end{array}$ & $\mathrm{n}$ & 73 & 51 & 15 \\
\hline & & $\mathrm{F}$ & $2.08(2.01-2.15)$ & $2.18(2.10-2.26)$ & $4.96(4.26-5.78)$ \\
\hline & & $\mathrm{R}$ & $2.48(2.23-2.76)$ & $2.44(2.17-2.73)$ & $4.58(3.33-6.30)$ \\
\hline & Cigarettes only & $\mathrm{n}$ & 8 & 2 & 2 \\
\hline & & $F$ & $6.38(5.61-7.24)$ & $3.03(2.29-4.01)$ & $6.69(3.43-13.05)$ \\
\hline & & $\mathrm{R}$ & $6.42(4.22-9.78)$ & $5.04(1.29-19.70)$ & $6.69(3.43-13.05)$ \\
\hline
\end{tabular}




\begin{tabular}{|c|c|c|c|c|c|}
\hline & Between levels & $P_{B}$ & $<0.001$ & $<0.05$ & $<0.01$ \\
\hline \multirow[t]{6}{*}{ Unexposed base ${ }^{c}$} & Never any product & $\mathrm{n}$ & 57 & 70 & 14 \\
\hline & & $\mathrm{F}$ & $2.95(2.82-3.09)$ & $2.61(2.52-2.69)$ & $2.75(2.47-3.06)$ \\
\hline & Never cigarettes & $\mathrm{n}$ & 72 & 44 & 14 \\
\hline & & $\mathrm{F}$ & $2.05(1.98-2.12)$ & $2.16(2.07-2.24)$ & $4.62(3.93-5.43)$ \\
\hline & & $\mathrm{R}$ & $2.47(2.22-2.74)$ & $2.42(2.15-2.73)$ & $4.28(3.08-5.96)$ \\
\hline & Between levels & $P_{B}$ & $<0.001$ & $<0.01$ & $<0.05$ \\
\hline \multirow[t]{6}{*}{ RR adjusted for age } & Yes & $\mathrm{n}$ & 69 & 63 & 15 \\
\hline & & $\mathrm{F}$ & $2.46(2.35-2.56)$ & $2.17(2.10-2.24)$ & $2.70(2.43-3.00)$ \\
\hline & & $\mathrm{R}$ & $2.97(2.56-3.45)$ & $2.46(2.26-2.69)$ & $4.44(2.90-6.82)$ \\
\hline & No & $n$ & 60 & 51 & 13 \\
\hline & & $\mathrm{F}$ & $2.26(2.18-2.34)$ & $2.78(2.67-2.88)$ & $4.91(4.17-5.79)$ \\
\hline & Between levels & $P_{B}$ & NS & $<0.001$ & $<0.05$ \\
\hline \multirow{7}{*}{$\begin{array}{l}\text { RR adjusted for factors other than age } \\
\text { or sex }\end{array}$} & Yes & $\mathrm{n}$ & 34 & 30 & 6 \\
\hline & & $\mathrm{F}$ & $2.09(1.98-2.21)$ & $2.28(2.20-2.37)$ & $2.11(1.87-2.38)$ \\
\hline & & $\mathrm{R}$ & $2.64(2.23-3.13)$ & $2.41(2.14-2.71)$ & $2.11(1.87-2.38)$ \\
\hline & No & $\mathrm{n}$ & 95 & 84 & 22 \\
\hline & & $\mathrm{F}$ & $2.43(2.35-2.51)$ & $2.53(2.45-2.61)$ & $5.26(4.62-6.00)$ \\
\hline & & $\mathrm{R}$ & $3.00(2.68-3.36)$ & $2.86(2.60-3.15)$ & $5.02(3.88-6.50)$ \\
\hline & Between levels & $P_{B}$ & $<0.1$ & $<0.1$ & $<0.001$ \\
\hline
\end{tabular}

${ }^{a}$ Within each study, results are selected in the following order or preference, within each sex, for: unexposed group-never any product, never cigarettes, other; smoking product-any, cigarettes (ignoring other products), cigarettes only; overlapping studies-principal, subsidiary; and then for single sex results in preference to combined sex results. Results adjusted for the most potential confounders are selected.

${ }^{b} \mathrm{n}=$ number of estimates combined, $\mathrm{F}=$ fixed-effect meta-analysis $\mathrm{RR}(95 \% \mathrm{Cl}), \mathrm{R}=$ random-effects meta-analysis $\mathrm{RR}(95 \% \mathrm{Cl}), \mathrm{H}=\mathrm{heterogeneity} \mathrm{chisquared} \mathrm{per}$ degree of freedom, $P_{H}=$ probability value for heterogeneity expressed as $p<0.001, p<0.05, p<0.1$ or NS ( $p \geq 0.1$ ), $P_{B}=$ probability value for between levels (see methods) similarly expressed.

' Includes acceptable near-equivalent estimate (see methods) if estimate for strictly defined never smoker base not available (COPD: 3 for never cigarettes, CB: 2 for never any product and 4 for never cigarettes).

Sex RRs for males are highest for all three outcomes, with the variation by sex significant for CB $(p<0.001)$ and emphysema $(\mathrm{p}<0.05)$.

Continent There is significant variation by continent for COPD ( $\mathrm{p}<0.001), \mathrm{CB}(\mathrm{p}<0.05)$ and emphysema ( $\mathrm{p}<$ $0.001)$. For COPD and CB, RRs are higher for North America than for Europe, Asia or other countries (including multicentre results). For emphysema, RRs are again relatively low for Europe, though RRs for North America and Asia are similar.

Publication year For all three outcomes, there is significant $(\mathrm{p}<0.001)$ variation by publication year. Though there is some indication that RRs are relatively high for studies published before 1980, the pattern is erratic for both COPD (high for pre-1980 and 1990-99, low for 1980-89 and 2000 or later) and emphysema (high for pre-1980 and 2000 or later, low for 1980-89 and 1990-99).

Study type For COPD, there is marked variation ( $<<$ 0.001), with RRs higher for prospective studies than for other study designs. For $\mathrm{CB}$, no variation is evident, most RRs coming from cross-sectional studies. For emphysema, where no results come from case-control studies, RRs are again higher in prospective studies, particularly for the fixed-effect estimates $(\mathrm{p}<0.05)$.

Outcome subtype For all three outcomes, the estimates are substantially higher when based on mortality, although for $\mathrm{CB}$ and emphysema few RRs are so based. For COPD, the random-effects estimates of 3.95 (3.00$5.21, \mathrm{n}=29)$ based on mortality, and $2.35(2.13-2.59, \mathrm{n}$ $=60$ ) based on lung function, differ substantially.

How asthma was taken into account For COPD, the random-effect estimates tend to be lower when asthmatics are excluded $(2.35,1.92-2.87, \mathrm{n}=21)$ or when asthma is included as part of the definition (2.55, $2.00-3.26, \mathrm{n}=19)$, than when it is ignored $(2.95,2.62$ $3.32, \mathrm{n}=76)$ or is taken into account in other ways or it is unclear whether the definition of the outcome includes asthma or not $(3.62,2.80-4.69, \mathrm{n}=13)$. For $\mathrm{CB}$ and emphysema, the great majority of RRs come from studies where the comparison is made irrespective of asthma. 
Study size There is no convincing evidence that RRs vary according to the number of cases of the outcome that are studied.

Analysis type For COPD, RRs based on onset are clearly higher than those based on prevalence ( $\mathrm{p}<$ $0.001)$. A similar tendency is seen for emphysema ( $\mathrm{p}<$ $0.05)$, though only six RRs are based on onset. For CB, where again nearly all RRs are based on prevalence, no difference is seen by analysis type.

Smoking product For COPD, a clear difference is seen by definition of smoking product $(\mathrm{p}<0.001)$, with random-effects estimates of $6.42(4.22-9.78, \mathrm{n}=8)$ for cigarette only smoking, $2.48(2.23-2.76, \mathrm{n}=73)$ for cigarettes ignoring other products, and 2.99 (2.61-3.44, $\mathrm{n}=48$ ) for any product. For CB and emphysema, RRs based on cigarette only smoking are few, and the pattern less clear.

Unexposed base group For COPD, RRs are lower when the comparison group is never cigarettes (so that smokers of other products only may be included in the denominator) than when it is never any product ( $\mathrm{p}<$ $0.001)$. For $C B$, there is a smaller difference in the same direction $(p<0.01)$. For emphysema, however, fixedeffect estimates are lower when the comparison group is never any product $(\mathrm{p}<0.05)$, but this difference is reversed when random-effects estimates are used.

Age-adjustment For COPD, there is no difference in RRs based on age-adjusted or age-unadjusted RRs. For CB ( $\mathrm{p}<0.001)$ and emphysema $(\mathrm{p}<0.05)$, however, adjusted RRs are lower.

Adjustment for factors other than age or sex For all three outcomes, there is a tendency for RRs adjusted for other factors to be lower than those that are not so adjusted ( $\mathrm{p}<0.1$ for COPD and $\mathrm{CB}, \mathrm{p}<0.001$ for emphysema).

Variation by other characteristics (see Additional file 10) was also studied. For no outcome is there any clear evidence that RRs varied by the type of tobacco (blended or Virginia) typically used in the country where the study was conducted, by the lowest, or highest, age of subjects included in the study, by presence of the study weaknesses defined in Table 2 , by whether the outcome was assessed using a bronchodilator (only relevant to COPD), or by whether the RR was directly available, derived from $2 \times 2$ tables provided, or using more complex methods. Differences are seen by start year of the study, but, like publication year, they do not follow any clear pattern over time. For emphysema, estimates are higher $(\mathrm{p}<0.001)$ for studies providing RRs only for ever smoking than studies providing RRs for both ever smoking and current smoking, with random-effects estimates, respectively, $5.51(4.08-7.43, \mathrm{n}=11)$ and 3.77 $(2.63-5.42, \mathrm{n}=17)$. Sexes combined RRs tend to be lower if adjusted for sex for COPD $(p<0.05)$ and emphysema ( $\mathrm{p}<0.001)$, but not for CB. RRs adjusted for more factors tend to be lower for COPD ( $<<0.01)$, $\mathrm{CB}(\mathrm{p}<0.01)$ and emphysema $(\mathrm{p}<0.001)$. This is unsurprising given the results already noted for adjustment for age and for factors other than age or sex.

For COPD, the relationship to the characteristics was also studied separately for three subtypes of outcomebased on mortality (31 RRs), on lung function (62 RRs) and on other definitions (42 RRs). The tendency for RRs to be higher for North American studies is clearest when outcome is based on mortality, also evident when based on lung function only, but not evident when based on other definitions. The relationship of risk to study type cannot usefully be studied as nearly all relevant mortality studies are prospective, and nearly all other studies are cross-sectional. Similarly most data from mortality studies are of onset, whereas most data from other studies are of prevalence. The higher RRs noted in the overall results for smoking of cigarettes only are also evident solely in the mortality studies, as no RRs for this exposure are included for the other COPD subtypes. There is, however, a consistent tendency for all subtypes for RRs to be higher when the comparison group is never smoking of any product than when it is never smoking of cigarettes.

As only three CB RRs based on mortality are included, the relationship to the characteristics for $\mathrm{CB}$ is only studied separately for two subtypes-outcomes based on symptoms ( $83 \mathrm{RRs})$, and other than on mortality or symptoms ( $28 \mathrm{RRs}$ ). Tendencies noted in Table 5 for RRs to be higher in males than females, lower if adjusted for age than if unadjusted, and lower if the unexposed base group is never cigarettes than if it is never any product, are apparent for both subtypes.

For emphysema, the relationship to the characteristics separated by subtype of outcome cannot usefully be studied due to limited numbers, with four of the 28 RRs being based on mortality, and 24 based on other definitions.

In an attempt to evaluate the independent role of the characteristics, meta-regression analyses were conducted for COPD and $\mathrm{CB}$, the results from the basic model being summarized in Table 6 . There are too few data points for emphysema for useful meta-regression analysis, especially since almost half the total weight comes from one study (LAVECC).

For COPD the deviance reduces from 1,038.1 on 128 degrees of freedom to 421.8 on 112 degrees of freedom on fitting the basic model, substantially reducing, but not eliminating, the heterogeneity. The results in Table 6 demonstrate an independent role of six characteristics noted in the univariate analyses: sex (lower RRs for females), continent (higher for North America), smoking product (higher for cigarette smokers than smokers of 
Table 6 Meta-regression results for ever smoking of any product (or cigarettes if any product not available) ${ }^{\mathrm{a}}$

\begin{tabular}{|c|c|c|c|c|c|}
\hline \multirow[b]{2}{*}{ Characteristic } & \multirow[b]{2}{*}{ Level } & \multicolumn{2}{|l|}{ COPD } & \multicolumn{2}{|l|}{$\mathrm{CB}$} \\
\hline & & Estimate $^{\mathrm{b}}\left(\mathrm{SE}^{\mathrm{c}}\right)$ & $p^{d}$ & Estimate $^{\mathrm{b}}\left(\mathrm{SE}^{\mathrm{c}}\right)$ & $p^{d}$ \\
\hline Constant & & $+1.149(0.141)$ & & $+1.316(0.266)$ & \\
\hline \multirow[t]{3}{*}{ Sex } & Male & Base & $<0.05$ & Base & $<0.1$ \\
\hline & Female & $-0.212(0.044)$ & & $-0.171(0.036)$ & \\
\hline & Combined & $-0.006(0.039)$ & & $-0.121(0.077)$ & \\
\hline \multirow[t]{4}{*}{ Continent } & N America & Base & $<0.05$ & Base & $<0.01$ \\
\hline & Europe & $-0.200(0.040)$ & & $-0.278(0.039)$ & \\
\hline & Asia & $-0.295(0.067)$ & & $-0.281(0.060)$ & \\
\hline & Other & $-0.355(0.072)$ & & $-0.177(0.074)$ & \\
\hline \multirow[t]{3}{*}{ Outcome subtype } & Mortality & Base & $<0.01$ & Base & NS \\
\hline & Lung function (COPD) or symptoms (CB) & $-0.404(0.092)$ & & $-0.212(0.249)$ & \\
\hline & Other & $-0.114(0.085)$ & & $-0.302(0.248)$ & \\
\hline \multirow[t]{4}{*}{ How asthma taken into account } & Ignored & Base & $<0.001$ & Base & $<0.05$ \\
\hline & Excluded & $-0.143(0.043)$ & & $-0.109(0.066)$ & \\
\hline & Included in outcome definition & $-0.461(0.060)$ & & No data & \\
\hline & Other & $+0.283(0.093)$ & & $+0.996(0.214)$ & \\
\hline \multirow[t]{3}{*}{ Smoking product } & Any & Base & $<0.01$ & Base & NS \\
\hline & Cigarettes (ignoring other products) & $+0.428(0.128)$ & & $-0.060(0.128)$ & \\
\hline & Cigarettes only & $+0.589(0.107)$ & & $+0.449(0.149)$ & \\
\hline \multirow[t]{2}{*}{ Unexposed group } & Never any product & Base & $<0.05$ & Base & NS \\
\hline & Never cigarettes & $-0.603(0.123)$ & & $-0.111(0.126)$ & \\
\hline \multirow[t]{2}{*}{ RR adjusted for age } & Yes & Base & NS & Base & $<0.01$ \\
\hline & No & $+0.046(0.045)$ & & $+0.214(0.032)$ & \\
\hline \multirow[t]{2}{*}{ RR adjusted for factor other than age or sex } & Yes & Base & $<0.1$ & Base & NS \\
\hline & No & $+0.195(0.052)$ & & $-0.113(0.070)$ & \\
\hline Midpoint age & Per 10 years & $+0.003(0.002)$ & NS & $+0.003(0.001)$ & NS \\
\hline
\end{tabular}

a Based on the same data as for Table 5. See that table for further definition of RRs selected for analysis, and numbers of estimates of each characteristic level.

${ }^{b}$ Estimates are of log RR. For a particular entry, the predicted RR for a given estimate is calculated by adding the constant to the values for each level applicable to the estimate (taking the value for the base level as zero) and taking the exponential of the result.

c $\mathrm{SE}=$ standard error.

${ }^{d}$ The $p$ value is estimated from the drop in deviance from removing the characteristic from the model using an F-test. It is expressed as $p<0.001, p<0.01, p<$ $0.05, p<0.1$ or NS $(p \geq 0.1)$.

any product), the unexposed base (higher for never any product than never cigarettes), and particularly the outcome subtype (lower when based on lung function), and the way asthma is taken into account (lowest when asthma is included in the COPD definition). Effects of adjustment and of age are not clearly seen, however. For none of the secondary characteristics do their inclusion into the model significantly improve the fit (at $\mathrm{p}<0.05$ ). This includes study type and analysis type, which are highly significant $(p<0.001)$ in the univariate analyses shown in Table 5 . Both these are highly correlated with outcome subtype-thus where mortality is the outcome, the study type will nearly always be prospective, and the analysis type will nearly always be onset.

Inspection of standardized residuals from the basic model for COPD reveals two estimates where the observed RR differ markedly from the RR fitted by the model. The largest residual of -3.49 is for males in study $\mathrm{XU}$, where an observed RR of 1.26 compares with a fitted RR of 2.20. The corresponding RRs for females are 2.61 observed and 1.78 fitted, with a residual of +1.12. For study GODTFR, sexes combined, the observed RR of 5.46 compares with a fitted value of 2.79 , with a residual of +2.58 . Other residuals are all less than $+/-2.20$.

For CB the deviance reduces from 657.1 on 113 degrees of freedom to 433.3 on 103 degrees of freedom on fitting the basic model, again substantially reducing, but not eliminating, the heterogeneity. Though the direction of differences by level of the various characteristics is quite similar to that for COPD, the effects of individual characteristics are less clear, with significant differences (at $\mathrm{p}<0.05)$ only for continent, how asthma was taken into account, and age-adjustment. No secondary characteristics help to improve the model fit (at $p<0.05$ ), except for publication year, where a tendency is seen for earlier published studies to provide higher RRs. 
The largest standardized residual from the basic model for $\mathrm{CB},-2.74$, is for males in study GOLDBE, where the observed RR of 1.48 compares to a fitted RR of 2.69, corresponding RRs for females being 2.87 observed and 2.27 fitted, with a residual of +0.79 . Another large residual, -2.53, is for females in study JOUSI1, where the observed RR of 1.66 compares to a fitted value of 2.43 , with the corresponding RRs for males being 2.42 observed and 2.88 fitted, with a residual of -1.36 . Other residuals are all less than $+/-2.20$.

\section{B. Risk from current smoking}

Figures 6 and 7 (COPD), 8 and 9 (CB) and 10 (emphysema) present the results of the main meta-analyses for current smoking of any product. As before, RRs for smoking of cigarettes are used if RRs for any product smoking are not available, and RRs are most-adjusted. Some results by levels of characteristics studied are shown in Table 7.

As for ever smoking, the RRs for COPD, CB and emphysema are heterogeneous $(\mathrm{p}<0.001)$, with the largest seen being 43.92 for COPD (DICKIN/sexes combined), 27.02 for CB (MILLER/females), and a remarkable 489.54, with lower 95\% CL 211.74, for emphysema (AUERBA/males). The random-effects estimates (COPD 3.51, 95\% CI 3.08-3.99, $\mathrm{n}=120$; CB, 3.41, 3.13-3.72, $\mathrm{n}=113$; emphysema $4.87,2.83-8.41, \mathrm{n}=22$ ) are all clearly positive, and somewhat larger than the corresponding estimates for ever smoking. Similarly to ever smoking, the individual RRs are virtually all above 1.0, though varying substantially. The estimates are also little affected by preferring RRs for current smoking of cigarettes to those for current smoking of any product, the random-effects estimates changing to 3.59 (3.154.09) for COPD, 3.45 (3.17-3.77) for CB and 5.00 (2.878.72) for emphysema. The estimates are again little affected by preferring least, rather than most, adjusted RRs, with the estimates now 3.41 (3.00-3.87) for COPD, 3.43 (3.12-3.77) for $\mathrm{CB}$ and $4.32(2.40-7.78)$ for emphysema.

For the main meta-analysis, the studies contributing the most to the total weight are the same as for the corresponding meta-analysis for ever smoking: ZIELI2/ females for COPD (11.7\% of the total of 4,226$)$, and LAVECC/sexes combined for CB $(11.4 \%$ of 4,326$)$ and emphysema (61.9\% of 287).

For the characteristics considered in Table 7 the pattern of variation seems quite similar to that for ever smoking in Table 5. Thus, as for ever smoking, RRs tend to be higher for males and for North American studies for all three outcomes, higher for prospective studies for COPD, and higher when based on mortality for COPD and $\mathrm{CB}$, with no obvious variation by study size, and an erratic pattern for publication year. RRs also show a similar pattern by how asthma is taken into account for COPD to that seen for ever smoking, and are again higher when based on onset for COPD, higher for cigarette only smoking for COPD, higher when the unexposed group is never smoked any product for COPD, and lower for RRs unadjusted for age for CB. As for ever smoking, variation in RRs by other characteristics (not shown in Table 7) was also studied. For most of these there seems little evidence of any difference. For COPD, there is a tendency $(p<0.001)$ for estimates to be higher for studies providing RRs only for current smoking than for studies providing RRs for both ever smoking and current smoking, with random-effects estimates, respectively, $4.52(2.69-7.59, \mathrm{n}=10)$ and 3.40 (3.00-3.87, $\mathrm{n}=110)$, but no such differences are seen for $\mathrm{CB}$ and emphysema. Compared to the results for ever smoking, there seems less clear evidence of an effect of adjustment, except as already noted for adjustment for age for $\mathrm{CB}$ (Table 7).

For COPD, the relationship to the characteristics was also studied separately for outcomes based on mortality (33 RRs), based only on lung function (58 RRs) and based on other definitions (36 RRs). As for ever smoking, risk is higher in North American studies when the outcome is based on mortality or lung function, but not when based on other definitions. Also as for ever smoking, and for reasons stated in the previous section, variation cannot usefully be studied by study type, or by analysis type (onset or prevalence), or in relation to smoking of cigarettes only. Again RRs are consistently higher for all the outcome subtypes when the comparison group is never smoking of any product than when it is never smoking of cigarettes.

As only four CB RRs based on mortality are included, the relationship to the characteristics for $\mathrm{CB}$ is only studied separately for outcomes based on symptoms (81 RRs) and based other than on mortality or symptoms (28 RRs). The tendency noted in Table 7 for RRs to be higher for North American studies is only evident when outcome is based on symptoms, but the tendency for RRs to be lower if adjusted for confounders seems evident in both groups.

As is the case for ever smoking, the relationship to the characteristics by outcome subtype cannot usefully be studied for emphysema due to limited numbers, with only four of 28 RRs based on mortality.

Also as for ever smoking, meta-regression analyses are conducted for $\mathrm{COPD}$ and $\mathrm{CB}$, the results from the basic model being summarized in Table 8 .

For COPD the deviance reduces from 1,643.4 on 119 degrees of freedom to 433.3 on 103 degrees of freedom on fitting the basic model. The results in Table 8 confirm the independent role of the six characteristics noted for ever smoking: sex, continent, smoking 


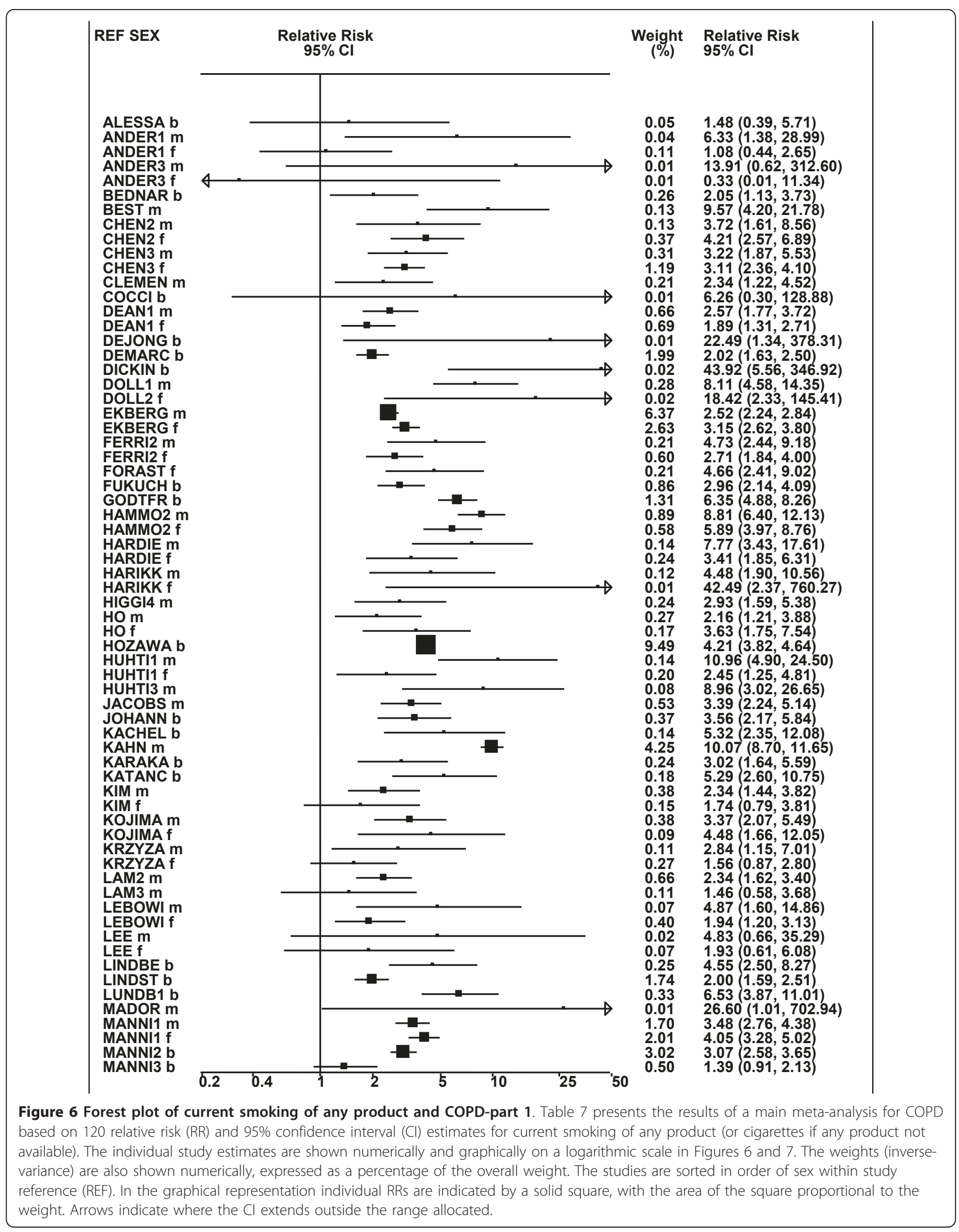




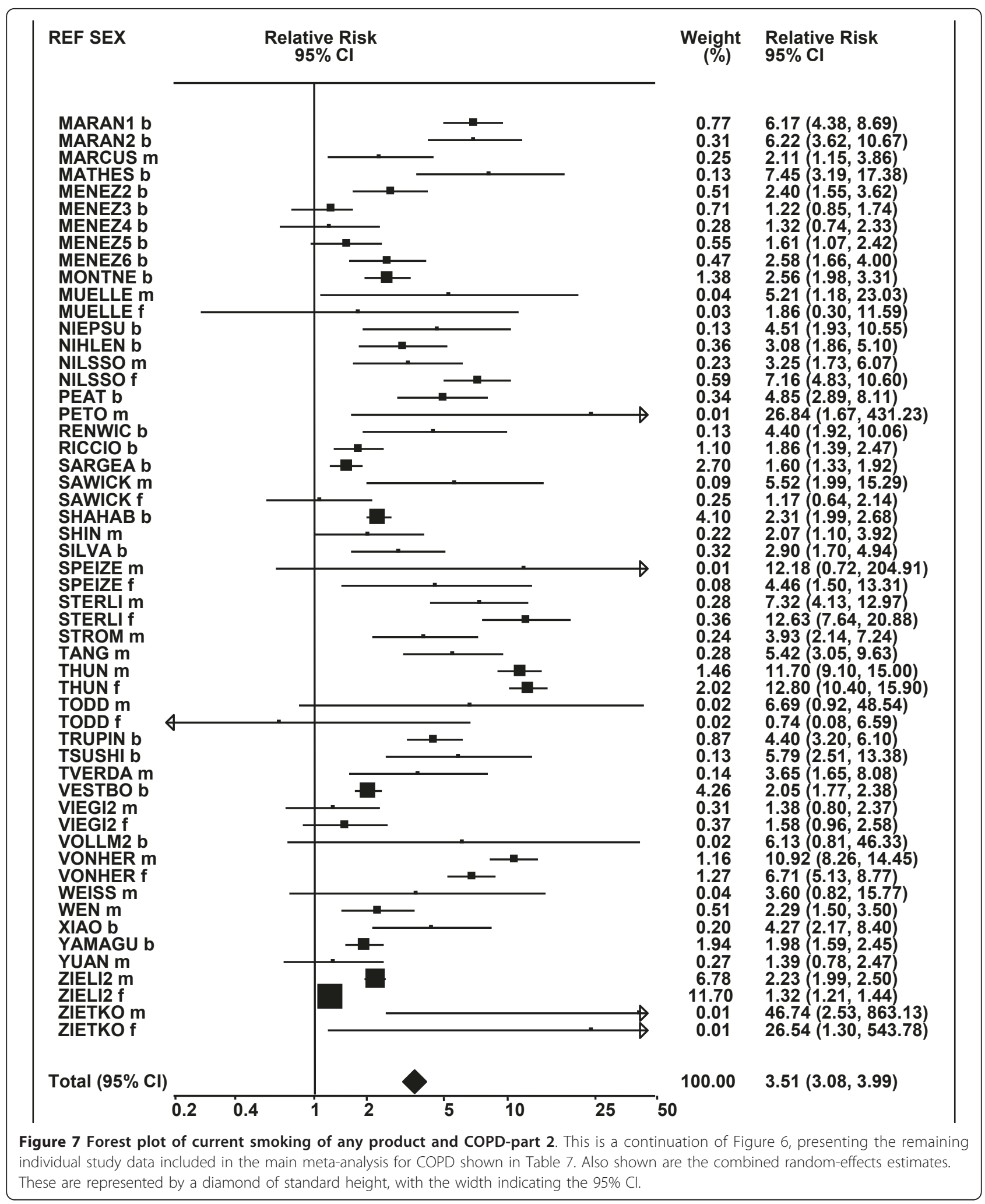




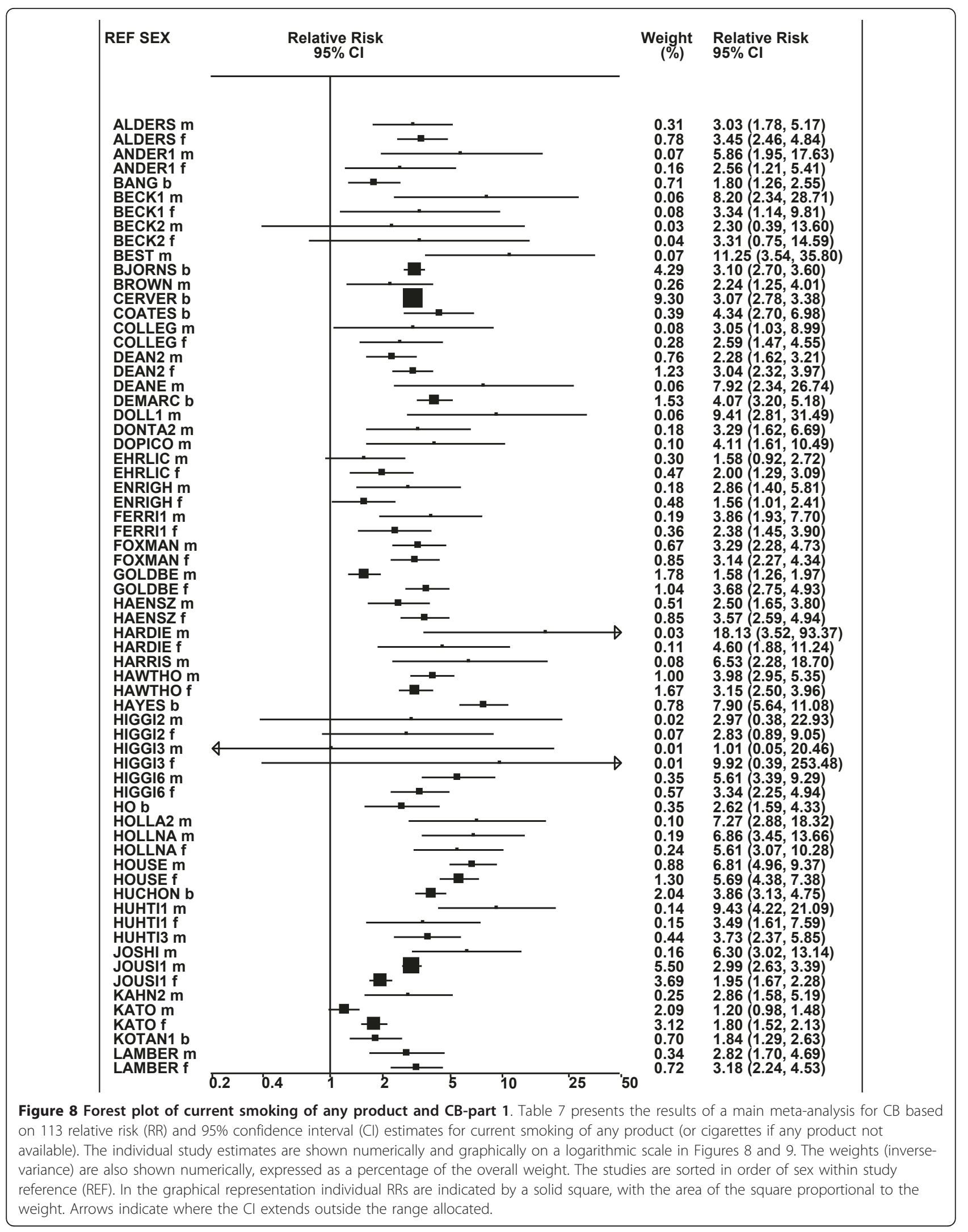




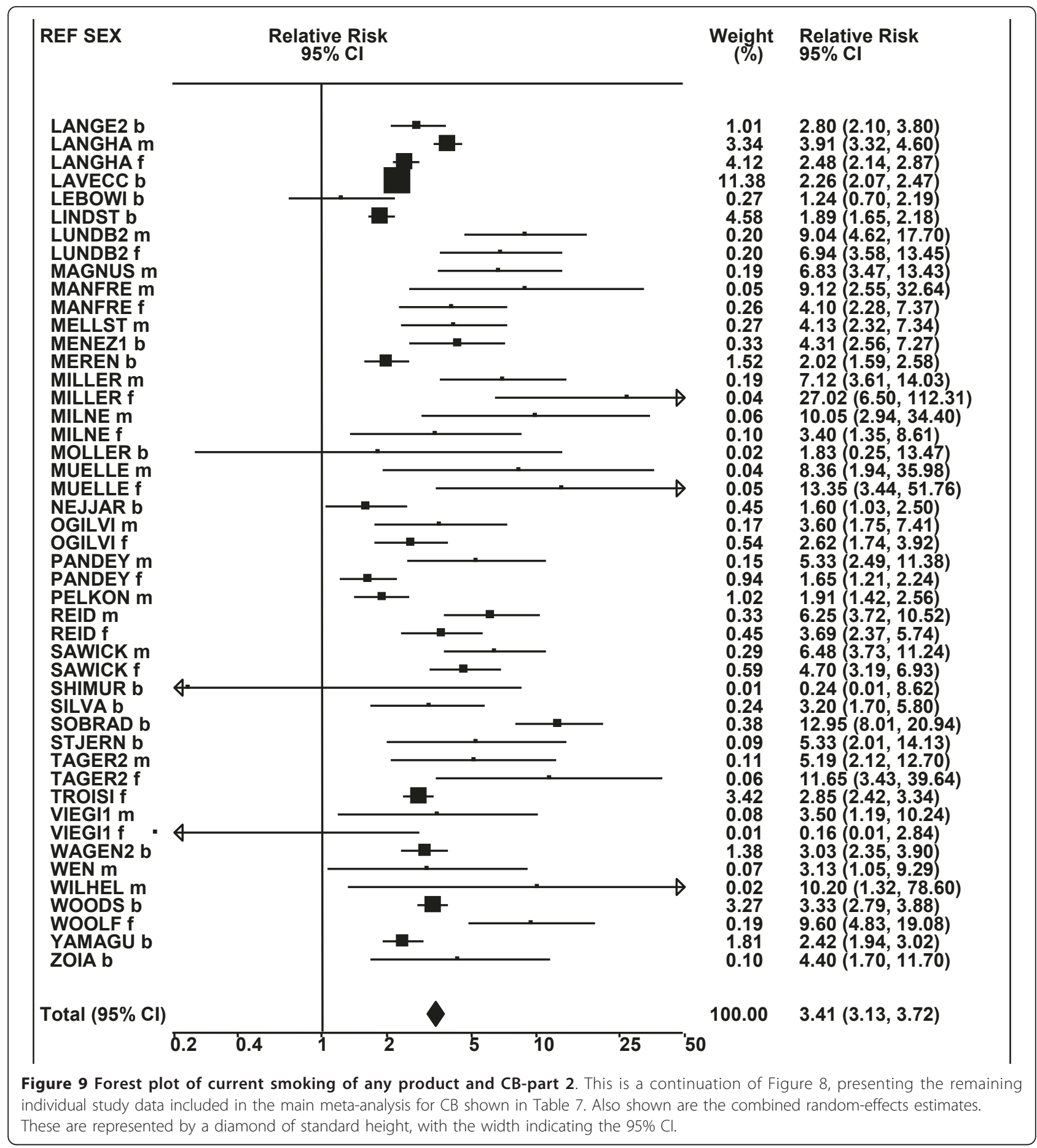

product, the unexposed group, outcome subtype, and the way asthma is taken into account. A significant effect ( $p<0.05$ ) of age is also seen. No secondary variable significantly improves the fit (at $\mathrm{p}<0.05$ ). The largest standardized residual from the basic model, +2.30 , is for males in study VONHER where the observed RR of 10.92 compares to a fitted RR of 5.58, corresponding
RRs for females being 6.71 observed and 4.49 fitted, with a residual of +1.44 .

For CB the deviance reduces from 649.2 on 112 degrees of freedom to 407.7 on 97 degrees of freedom on fitting the basic model. The characteristics which significantly (at $\mathrm{p}<0.05$ ) contribute to the model are continent, outcome subtype, whether the RR is adjusted for 


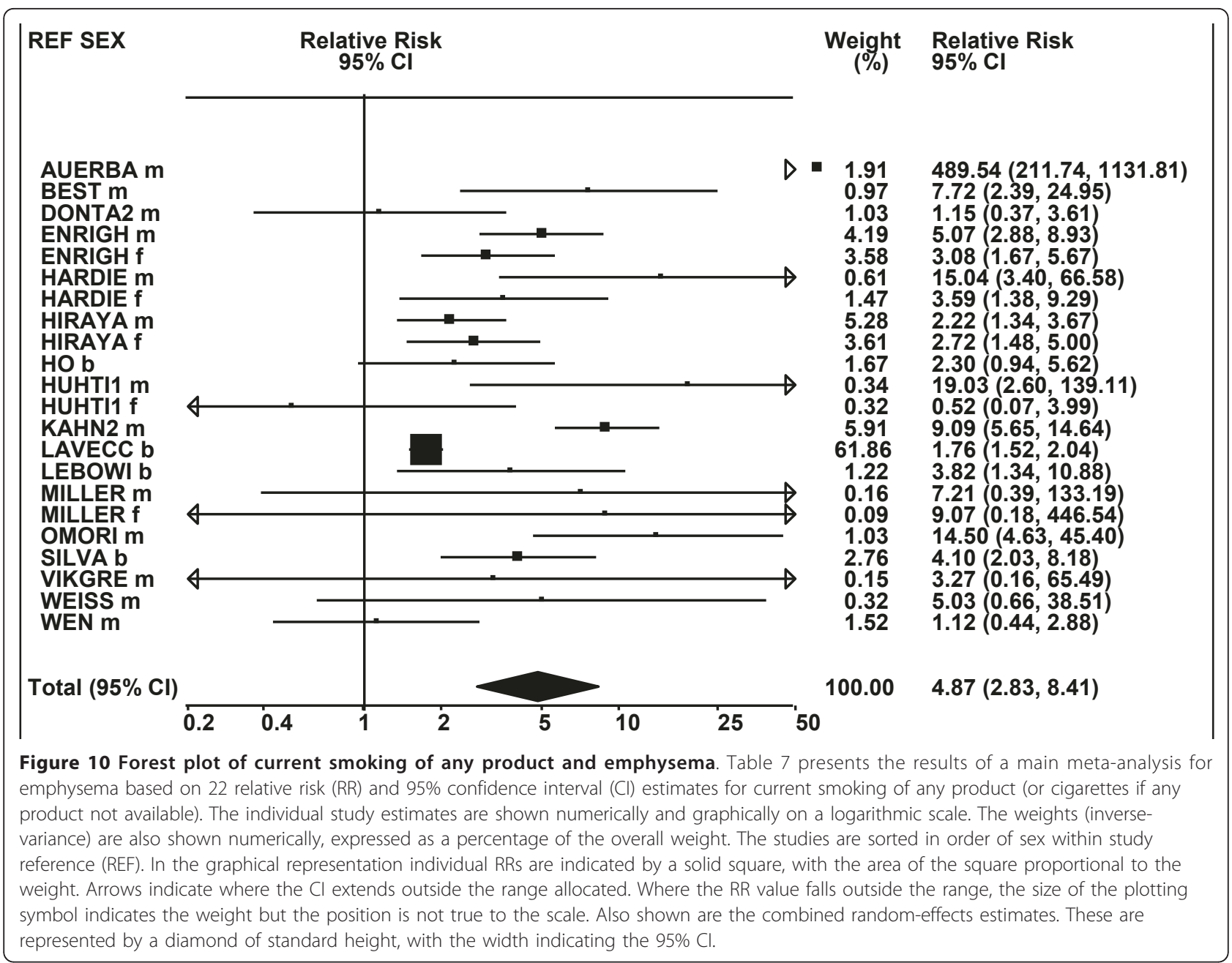

age, and how asthma is taken into account. As for ever smoking, the largest standardized residuals are for males in study GOLDBE (-3.44) and females in study JOUSI1 $(-2.88)$.

\section{Risk from ever or current smoking}

In an attempt to incorporate data from all the studies (except those with only dose-related data), additional analyses were carried out. The first set of analyses uses results for ever smoking if available from a study, but if not results for current smoking. Conversely, the second set prefers results for current smoking to results for ever smoking where both are available. The meta-analysis RRs are shown in Table 9. The RRs are intermediate between those for ever smoking (Table 5) and those for current smoking (Table 7). For example for COPD, random-effects estimates are 2.89 (95\%CI 2.63-3.17) specifically for ever smoking, 3.00 (2.71-3.32) preferring ever smoking, 3.46 (3.07-3.90) preferring current smoking, and 3.51 (3.08-3.99) specifically for current smoking. As so many of the RRs are common between the specific ever smoking analyses in Table 5 and the analyses preferring ever smoking in Table 9 the pattern of RRs by level of the characteristics studied tends to be quite similar. The same is true for the specific current smoking analyses and the analyses preferring current smoking in Table 9. Results for ever or current smoking by level of selected characteristics are therefore only presented in the Additional files.

\section{Risk from ex smoking}

Figures 11 and 12 (COPD), 13 and 14 (CB) and 15 (emphysema) present the results of the main meta-analyses for ex smoking of any product (or cigarettes if any product was not available), based on most-adjusted RRs. Some results by levels of characteristics are shown in Table 10

Again the RRs are markedly heterogeneous $(p<0.001$ for all three outcomes), ranging up to 55.86 for COPD (ANDER3/males), 96.60 for CB (MOLLER/combined sexes) and 12.50 for emphysema (BEST/males). The random-effects estimates (COPD 2.35, 95\% CI 2.11-2.63, n 
Table 7 Main meta-analyses for current smoking of any product (or cigarettes, if any product not available) ${ }^{\mathrm{a}}$

\begin{tabular}{|c|c|c|c|c|c|}
\hline Characteristic & Level & Statistic $^{\mathrm{b}}$ & COPD & CB & Emphysema \\
\hline \multirow[t]{4}{*}{$\overline{\text { All }}$} & All & $N$ & 120 & 113 & 22 \\
\hline & & $\mathrm{F}$ & $3.00(2.91-3.09)$ & $2.82(2.74-2.90)$ & $2.61(2.33-2.93)$ \\
\hline & & $\mathrm{R}$ & 3.51 (3.08-3.99) & $3.41(3.13-3.72)$ & $4.87(2.83-8.41)$ \\
\hline & & $\mathrm{H}, \mathrm{P}_{\mathrm{H}}$ & $13.81,<0.001$ & $5.80,<0.001$ & $11.54,<0.001$ \\
\hline \multirow[t]{10}{*}{ Sex } & Male & $N$ & 48 & 51 & 13 \\
\hline & & $\mathrm{F}$ & $3.80(3.60-4.02)$ & $3.08(2.90-3.28)$ & $6.71(5.29-8.53)$ \\
\hline & & $\mathrm{R}$ & $4.11(3.28-5.15)$ & $4.07(3.44-4.83)$ & $7.66(3.00-19.61)$ \\
\hline & Female & $n$ & 31 & 37 & 5 \\
\hline & & $\mathrm{F}$ & $2.53(2.39-2.68)$ & $2.75(2.60-2.90)$ & $2.85(1.94-4.18)$ \\
\hline & & $\mathrm{R}$ & $3.28(2.35-4.58)$ & $3.23(2.80-3.72)$ & $2.85(1.94-4.18)$ \\
\hline & Combined & $\mathrm{n}$ & 41 & 25 & 4 \\
\hline & & $\mathrm{F}$ & $2.81(2.69-2.95)$ & $2.74(2.62-2.86)$ & $1.86(1.62-2.14)$ \\
\hline & & $\mathrm{R}$ & $3.04(2.60-3.55)$ & $2.98(2.57-3.47)$ & $2.54(1.54-4.20)$ \\
\hline & Between levels & $P_{B}$ & $<0.05$ & NS & $<0.05$ \\
\hline \multirow[t]{13}{*}{ Continent } & N America & $n$ & 39 & 40 & 10 \\
\hline & & $\mathrm{F}$ & $5.02(4.76-5.29)$ & $3.44(3.20-3.69)$ & $8.13(6.32-10.45)$ \\
\hline & & R & $4.56(3.69-5.62)$ & $4.11(3.41-4.97)$ & $8.99(3.34-24.26)$ \\
\hline & Europe & $n$ & 55 & 58 & 7 \\
\hline & & $\mathrm{F}$ & $2.31(2.22-2.41)$ & $2.79(2.69-2.89)$ & $1.83(1.58-2.11)$ \\
\hline & & $\mathrm{R}$ & $3.31(2.80-3.92)$ & $3.28(2.96-3.62)$ & $2.88(1.36-6.09)$ \\
\hline & Asia & $\mathrm{n}$ & 17 & 9 & 5 \\
\hline & & $\mathrm{F}$ & $2.73(2.45-3.05)$ & $1.83(1.65-2.02)$ & $2.52(1.83-3.47)$ \\
\hline & & $\mathrm{R}$ & $2.86(2.27-3.60)$ & $2.26(1.67-3.05)$ & $2.74(1.50-4.99)$ \\
\hline & $\begin{array}{l}\text { Other or } \\
\text { multicountry }\end{array}$ & $n$ & 9 & 6 & 0 \\
\hline & & $\mathrm{F}$ & $2.15(1.89-2.44)$ & $3.32(2.94-3.75)$ & \\
\hline & & $\mathrm{R}$ & $2.37(1.75-3.21)$ & $3.11(2.33-4.16)$ & \\
\hline & Between levels & $P_{B}$ & $<0.001$ & $<0.001$ & $<0.01$ \\
\hline \multirow[t]{13}{*}{ Publication year } & Before 1980 & $n$ & 24 & 50 & 7 \\
\hline & & $\mathrm{F}$ & $5.64(5.12-6.21)$ & $3.59(3.36-3.84)$ & $15.01(10.59-21.28)$ \\
\hline & & $\mathrm{R}$ & $3.81(2.64-5.50)$ & $3.96(3.41-4.59)$ & $10.21(2.09-49.96)$ \\
\hline & 1980-89 & $n$ & 11 & 26 & 6 \\
\hline & & $\mathrm{F}$ & $2.18(1.84-2.59)$ & $2.29(2.16-2.44)$ & $1.83(1.59-2.10)$ \\
\hline & & $\mathrm{R}$ & $2.47(1.87-3.27)$ & $3.25(2.67-3.96)$ & $1.83(1.59-2.10)$ \\
\hline & 1990-99 & $n$ & 18 & 17 & 3 \\
\hline & & $\mathrm{F}$ & $6.20(5.59-6.88)$ & $2.74(2.56-2.93)$ & $3.65(2.50-5.31)$ \\
\hline & & $\mathrm{R}$ & $4.91(3.37-7.16)$ & $3.04(2.51-3.68)$ & $3.57(2.30-5.55)$ \\
\hline & 2000 or later & $\mathrm{n}$ & 67 & 20 & 6 \\
\hline & & $\mathrm{F}$ & $2.60(2.52-2.69)$ & $2.86(2.72-3.01)$ & $4.04(2.65-6.16)$ \\
\hline & & $\mathrm{R}$ & $3.13(2.73-3.60)$ & $2.94(2.50-3.47)$ & $4.57(2.05-10.20)$ \\
\hline & Between levels & $P_{B}$ & $<0.001$ & $<0.001$ & $<0.01$ \\
\hline \multirow[t]{10}{*}{ Study type } & Case-control & $n$ & 14 & 9 & 0 \\
\hline & & $\mathrm{F}$ & $3.72(3.09-4.48)$ & $3.39(2.90-3.96)$ & \\
\hline & & $\mathrm{R}$ & $4.69(2.83-7.77)$ & $3.66(2.77-4.83)$ & \\
\hline & Prospective & $n$ & 38 & 12 & 8 \\
\hline & & $\mathrm{F}$ & $6.46(6.01-6.95)$ & $2.96(2.67-3.29)$ & $3.61(2.81-4.64)$ \\
\hline & & $\mathrm{R}$ & $4.34(3.38-5.56)$ & $3.12(2.56-3.78)$ & $3.12(1.74-5.60)$ \\
\hline & Cross-sectional & $n$ & 68 & 92 & 14 \\
\hline & & $\mathrm{F}$ & $2.53(2.45-2.62)$ & $2.78(2.70-2.87)$ & $2.39(2.10-2.73)$ \\
\hline & & $\mathrm{R}$ & $3.00(2.63-3.41)$ & $3.42(3.10-3.77)$ & $6.60(2.74-15.92)$ \\
\hline & Between levels & $P_{B}$ & $<0.001$ & NS & NS \\
\hline Outcome subtype & Mortality & $\mathrm{n}$ & 31 & 4 & 5 \\
\hline
\end{tabular}


Table 7 Main meta-analyses for current smoking of any product (or cigarettes, if any product not available) ${ }^{a}$ (Continued)

\begin{tabular}{|c|c|c|c|c|c|}
\hline & & $\mathrm{F}$ & $6.57(6.09-7.10)$ & $4.16(2.67-6.48)$ & $3.79(2.87-5.00)$ \\
\hline & & $\mathrm{R}$ & $4.57(3.39-6.15)$ & $4.96(2.43-10.12)$ & $3.42(1.60-7.35)$ \\
\hline & $\begin{array}{l}\text { Lung function } \\
\text { (COPD) or symptoms } \\
\text { (CB) }\end{array}$ & $n$ & 56 & 81 & 0 \\
\hline & & F & $2.41(2.32-2.50)$ & $3.06(2.95-3.17)$ & \\
\hline & & R & $2.79(2.42-3.20)$ & $3.63(3.29-3.99)$ & \\
\hline & Other & $n$ & 33 & 28 & 17 \\
\hline & & $\mathrm{F}$ & $3.45(3.21-3.70)$ & $2.24(2.12-2.37)$ & $2.42(2.13-2.74)$ \\
\hline & & $\mathrm{R}$ & $3.77(3.01-4.72)$ & $2.69(2.26-3.21)$ & $5.56(2.65-11.68)$ \\
\hline & Between levels & $P_{B}$ & $<0.001$ & $<0.001$ & NS \\
\hline \multirow{13}{*}{$\begin{array}{l}\text { How asthma taken } \\
\text { into account }\end{array}$} & Ignored & $n$ & 72 & 103 & 19 \\
\hline & & $\mathrm{F}$ & $3.78(3.64-3.92)$ & $2.80(2.72-2.89)$ & $2.59(2.30-2.91)$ \\
\hline & & $\mathrm{R}$ & $3.60(3.08-4.20)$ & $3.38(3.08-3.70)$ & $5.02(2.80-9.00)$ \\
\hline & Excluded & $n$ & 17 & 6 & 3 \\
\hline & & $\mathrm{F}$ & 1.79 (1.69-1.90) & $2.85(2.53-3.21)$ & $4.03(1.85-8.77)$ \\
\hline & & $\mathrm{R}$ & $3.05(2.34-3.97)$ & $3.30(2.39-4.54)$ & $3.90(0.57-26.55)$ \\
\hline & $\begin{array}{l}\text { Included in outcome } \\
\text { definition }\end{array}$ & $n$ & 18 & - & - \\
\hline & & $\mathrm{F}$ & $2.11(1.88-2.37)$ & & \\
\hline & & $\mathrm{R}$ & $2.73(2.10-3.55)$ & & \\
\hline & Other & $n$ & 13 & 4 & 0 \\
\hline & & $\mathrm{F}$ & $4.00(3.42-4.67)$ & $6.93(4.39-10.92)$ & \\
\hline & & $\mathrm{R}$ & $4.03(2.96-5.48)$ & $5.70(2.63-12.37)$ & \\
\hline & Between levels & $\mathrm{P}_{\mathrm{B}}$ & $<0.001$ & NS & NS \\
\hline \multirow[t]{13}{*}{ Number of cases } & $1-50$ & $n$ & 21 & 18 & 8 \\
\hline & & $\mathrm{F}$ & $4.62(3.42-6.24)$ & $3.85(2.81-5.28)$ & $2.09(1.31-3.34)$ \\
\hline & & $\mathrm{R}$ & $4.90(3.34-7.18)$ & $3.85(2.81-5.28)$ & $2.09(1.31-3.34)$ \\
\hline & $51-100$ & $n$ & 23 & 14 & 6 \\
\hline & & $F$ & $3.08(2.64-3.60)$ & $4.92(3.84-6.30)$ & $4.83(3.06-7.61)$ \\
\hline & & $\mathrm{R}$ & $3.21(2.48-4.16)$ & $5.28(3.88-7.03)$ & $5.14(2.53-10.45)$ \\
\hline & $101-200$ & $\mathrm{n}$ & 29 & 19 & 1 \\
\hline & & F & $2.61(2.35-2.89)$ & $3.93(3.41-4.52)$ & $14.50(4.63-45.41)$ \\
\hline & & $\mathrm{R}$ & $2.92(2.39-3.57)$ & $4.71(3.37-6.57)$ & $14.50(4.63-45.41)$ \\
\hline & $201+$ & $n$ & 47 & 62 & 7 \\
\hline & & F & $3.02(2.92-3.12)$ & $2.74(2.66-2.83)$ & $2.48(2.19-2.81)$ \\
\hline & & $\mathrm{R}$ & $3.66(3.02-4.43)$ & $3.06(2.78-3.27)$ & $6.62(2.44-18.00)$ \\
\hline & Between levels & $P_{B}$ & NS & $<0.05$ & NS \\
\hline \multirow[t]{7}{*}{ Analysis type } & Prevalence & $n$ & 84 & 103 & 14 \\
\hline & & $F$ & $2.57(2.48-2.65)$ & $2.82(2.74-2.91)$ & $2.39(2.10-2.73)$ \\
\hline & & $\mathrm{R}$ & $3.14(2.78-3.56)$ & $3.44(3.14-3.77)$ & $6.60(2.74-15.92)$ \\
\hline & Onset & $n$ & 36 & 10 & 8 \\
\hline & & $\mathrm{F}$ & $6.58(6.11-7.09)$ & $2.75(2.42-3.13)$ & $3.61(2.81-4.64)$ \\
\hline & & $\mathrm{R}$ & $4.40(3.41-5.69)$ & $3.00(2.31-3.89)$ & $3.12(1.74-5.60)$ \\
\hline & Between levels & $P_{B}$ & $<0.001$ & NS & NS \\
\hline \multirow[t]{6}{*}{ Smoking product } & Any & $n$ & 46 & 57 & 5 \\
\hline & & $\mathrm{F}$ & $3.09(2.92-3.26)$ & $2.84(2.72-2.96)$ & $2.05(1.78-2.36)$ \\
\hline & & $\mathrm{R}$ & $3.67(3.05-4.40)$ & $3.54(3.17-3.97)$ & $3.84(1.20-12.22)$ \\
\hline & $\begin{array}{l}\text { Cigarettes (ignoring } \\
\text { other products) }\end{array}$ & $n$ & 66 & 52 & 16 \\
\hline & & F & $2.67(2.57-2.77)$ & $2.79(2.67-2.91)$ & $4.37(3.54-5.40)$ \\
\hline & & $\mathrm{R}$ & $3.06(2.60-3.60)$ & $3.27(2.84-3.76)$ & $5.11(2.45-10.65)$ \\
\hline
\end{tabular}


Table 7 Main meta-analyses for current smoking of any product (or cigarettes, if any product not available) ${ }^{\mathrm{a}}$ (Continued)

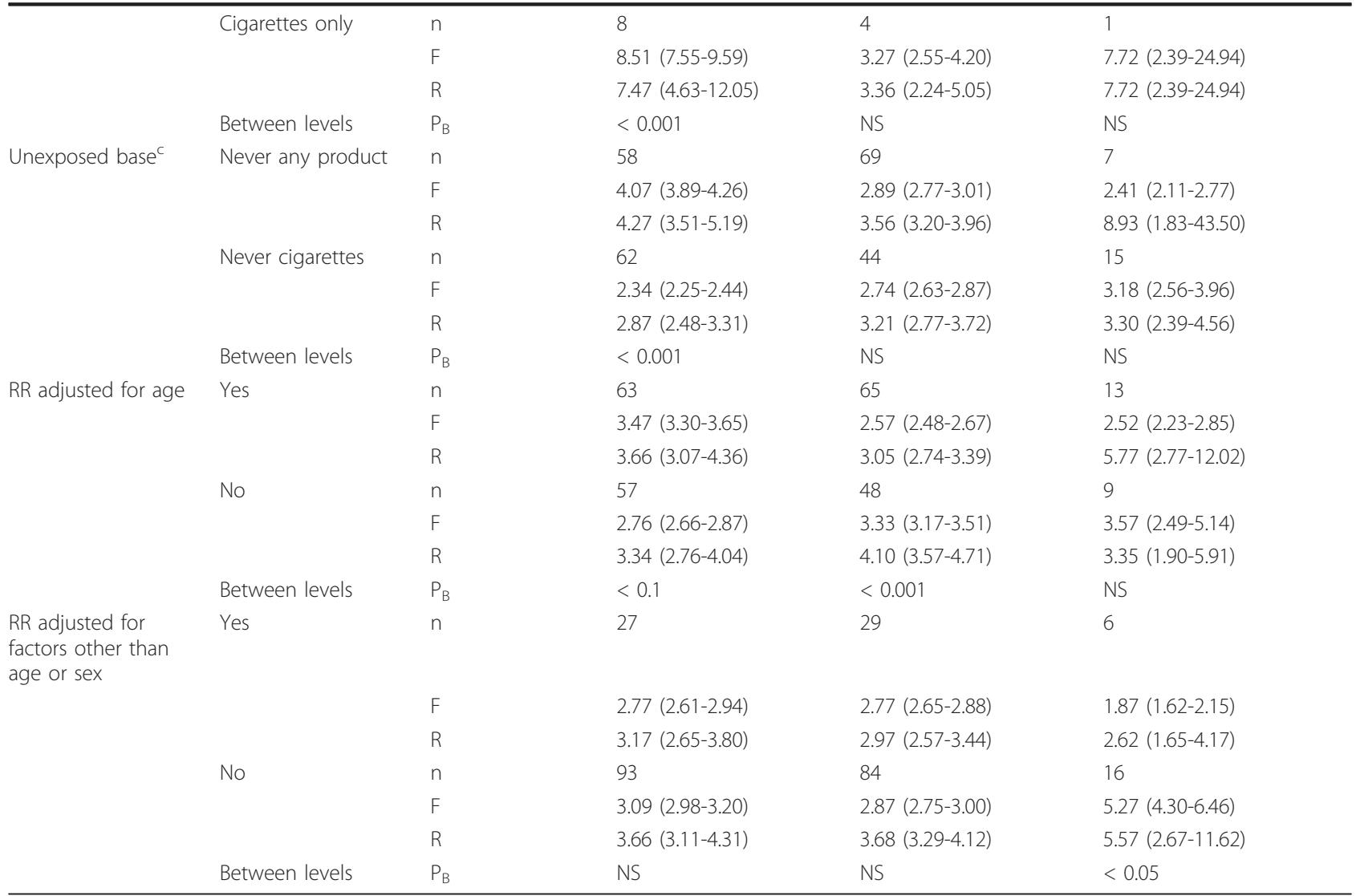

${ }^{a}$ Within each study, results are selected in the following order or preference, within each sex, for: unexposed group-never any product, never cigarettes, other; smoking product-any, cigarettes (ignoring other products), cigarettes only; overlapping studies-principal, subsidiary; and then for single sex results in preference to combined sex results. Results adjusted for the most potential confounders are selected.

${ }^{b} \mathrm{n}=$ number of estimates combined, $\mathrm{F}=$ fixed-effect meta-analysis $\mathrm{RR}(95 \% \mathrm{Cl}), \mathrm{R}=$ random-effects meta-analysis $\mathrm{RR}(95 \% \mathrm{Cl}), \mathrm{H}=\mathrm{heterogeneity} \mathrm{chisquared} \mathrm{per}$ degree of freedom, $P_{H}=$ probability value for heterogeneity expressed as $p<0.001, p<0.05, p<0.1$ or NS ( $p \geq 0.1$ ), $P_{B}=$ probability value for between levels (see methods) similarly expressed.

' Includes acceptable near-equivalent estimate (see methods) if estimate for strictly defined never smoker base not available (COPD: 3 for never cigarettes, CB: 2 for never any product and 4 for never cigarettes).

$=110 ; \mathrm{CB} 1.63,1.50-1.78, \mathrm{n}=105$; emphysema 3.52, 2.51-4.94, $\mathrm{n}=17$ ), though all clearly positive, are smaller than the corresponding estimates for current smoking. Individual RRs are only occasionally below 1.0 and never significantly so. Estimates are little affected by preferring RRs for ever smoking cigarettes to those for ever smoking any product, the random-effects estimates changing to 2.37 (2.12-2.64) for COPD and unchanged for $\mathrm{CB}$ and for emphysema. They are little changed by preferring least-adjusted RRs, with the estimates now 2.37 (2.13-2.63) for COPD, 1.72 (1.55-1.92) for CB and 3.68 (2.70-5.00) for emphysema.

For the main meta-analysis, the studies contributing the greatest to the total weight are ZIELI2/females for COPD (11.9\% of the total of 3,510), and LAVECC/sexes combined for CB $(13.1 \%$ of 2,493$)$ and emphysema (48.4\% of 300$)$.
For the characteristics considered in Table 10, the pattern of variation is quite similar to that for current smoking seen in Table 7. Thus, for COPD, RRs are, for both current and ex smoking, higher in males, lower in European studies, lower in cross-sectional studies, higher where the outcome is mortality, higher for cigarette only smoking and higher for never any product as the unexposed base. For $\mathrm{CB}$, RRs are higher for mortality for both current and ex smoking, but the differences by continent seen for current smoking are not evident for ex smoking. The same is true for differences by age-adjustment (not shown in Table 10). The small numbers of emphysema RRs for ex smoking (17) preclude reliable study of variation by level of the characteristics of interest. Further details of variations in RRs by level of the characteristics for all three outcomes, overall and (for COPD and CB) by outcome subtype are given in the Additional files. 
Table 8 Meta-regression analyses for current smoking of any product (or cigarettes if any product not available) ${ }^{\mathrm{a}}$

\begin{tabular}{|c|c|c|c|c|c|}
\hline \multirow[b]{2}{*}{ Characteristic } & \multirow[b]{2}{*}{ Level } & \multicolumn{2}{|l|}{ COPD } & \multicolumn{2}{|l|}{$\mathrm{CB}$} \\
\hline & & Estimate $^{\mathrm{b}}\left(\mathrm{SE}^{\mathrm{c}}\right)$ & $p^{d}$ & Estimate $^{\mathrm{b}}\left(\mathrm{SE}^{\mathrm{c}}\right)$ & $p^{d}$ \\
\hline Constant & & $+1.011(0.156)$ & & $+1.335(0.258)$ & \\
\hline \multirow[t]{3}{*}{ Sex } & Male & Base & $<0.05$ & Base & NS \\
\hline & Female & $-0.218(0.045)$ & & $-0.097(0.043)$ & \\
\hline & Combined & $-0.007(0.045)$ & & $+0.039(0.082)$ & \\
\hline \multirow[t]{4}{*}{ Continent } & N America & Base & $<0.001$ & Base & $<0.001$ \\
\hline & Europe & $-0.347(0.051)$ & & $-0.353(0.052)$ & \\
\hline & Asia & $-0.499(0.079)$ & & $-0.493(0.070)$ & \\
\hline & Other & $-0.510(0.081)$ & & $-0.144(0.086)$ & \\
\hline \multirow[t]{3}{*}{ Disease definition } & Mortality & Base & $<0.001$ & Base & $<0.01$ \\
\hline & Lung function (COPD) or symptoms (CB) & $-0.435(0.071)$ & & $-0.104(0.232)$ & \\
\hline & Other & $+0.049(0.076)$ & & $-0.406(0.232)$ & \\
\hline \multirow[t]{4}{*}{ How asthma taken into account } & Ignored & Base & $<0.001$ & Base & $<0.05$ \\
\hline & Excluded & $-0.334(0.051)$ & & $+0.159(0.076)$ & \\
\hline & Included in outcome definition & $-0.721(0.072)$ & & No data & \\
\hline & Other & $-0.055(0.103)$ & & $+1.225(0.237)$ & \\
\hline \multirow[t]{3}{*}{ Smoking product } & Any & Base & $<0.05$ & Base & NS \\
\hline & Cigarettes (ignoring other products) & $+0.255(0.084)$ & & $-0.023(0.136)$ & \\
\hline & Cigarettes only & $+0.505(0.099)$ & & $+0.410(0.136)$ & \\
\hline \multirow[t]{2}{*}{ Unexposed group } & Never any product & Base & $<0.01$ & Base & NS \\
\hline & Never cigarettes & $-0.446(0.077)$ & & $-0.127(0.130)$ & \\
\hline \multirow[t]{2}{*}{ RR adjusted for age } & Yes & Base & NS & Base & $<0.01$ \\
\hline & No & $+0.076(0.050)$ & & $+0.219(0.039)$ & \\
\hline \multirow[t]{2}{*}{ RR adjusted for factor other than age or sex } & Yes & Base & NS & Base & NS \\
\hline & No & $+0.148(0.057)$ & & $-0.007(0.072)$ & \\
\hline Midpoint age & Per 10 years & $+0.012(0.002)$ & $<0.05$ & $+0.003(0.002)$ & NS \\
\hline
\end{tabular}

a Based on the same data as for Table 7. See that table for further definition of RRs selected for analysis, and numbers of estimates of each characteristic level.

${ }^{b}$ Estimates are of log RR. For a particular entry, the predicted RR for a given estimate is calculated by adding the constant to the values for each level applicable to the estimate (taking the value for the base level as zero) and taking the exponential of the result.

c $\mathrm{SE}=$ standard error.

${ }^{d}$ The $p$ value is estimated from the drop in deviance from removing the characteristic from the model using an F-test. It is expressed as $p<0.001, p<0.01, p<$ $0.05, p<0.1$ or NS $(p \geq 0.1)$.

\section{E. Risk by amount smoked}

Table 11 summarizes the results of meta-analyses using RRs categorized by amount smoked. These are based on those 33 studies for COPD, 44 for CB and 10 for emphysema which provided data that could be used in the meta-analyses. For all three outcomes, results are shown for one of the sets of "key values" (see methods) and for the comparison of the highest and lowest exposures. For all three outcomes, a clear increase is seen for RRs for categories containing 5, but not 20, cigarettes/day, with the meta-analysis RR increasing monotonically with increasing amount smoked. Randomeffects estimates for categories containing 45, but not 20 cigarettes/day, are $9.50(7.38-12.22, \mathrm{n}=26)$ for COPD, 7.37 (5.86-9.28, $\mathrm{n}=35)$ for $\mathrm{CB}$ and 7.19 (2.74$18.87, \mathrm{n}=6$ ) for emphysema. The key value analyses do not use results for all the dose-response data available, as a number of the studies use broad doseresponse categories (such as 1-20 or $20+$ cigs/day) which span more than one of the key values. The "highest vs. lowest" analyses in Table 11 include results from all the dose response relationships which can be meta-analysed, and emphasise the positive relationship, with random-effects estimates of 2.32 (1.90-2.83, $\mathrm{n}=$ 44) for COPD, $2.42(2.10-2.79, \mathrm{n}=62)$ for $C B$, and 2.73 (1.23-6.04) for emphysema. Fuller results are given in the Additional files. These results, which also include some meta-analyses by level of selected characteristics, show that the increase with amount smoked is also clearly evident using an alternative set of key values $(1,10,20,30,40,999)$, though numbers of available RRs are quite sparse for the higher values, and using least-adjusted rather than most-adjusted RRs. The additional files also include available results for some other studies which present dose response data in a form that cannot readily be included in the meta-analyses (e.g. comparison of mean or median consumption in cases and non-cases). These results do 
Table 9 Main meta-analyses for current or ever smoking of any product (or cigarettes, if not available)a

\begin{tabular}{lllll}
\hline Preference & Statistic $^{\mathbf{b}}$ & COPD & CB & Emphysema \\
\hline Ever smoking to current smoking & $\mathrm{n}$ & 138 & 121 & 33 \\
& $\mathrm{~F}$ & $2.47(2.40-2.53)$ & $2.42(2.36-2.48)$ & $3.32(3.04-3.61)$ \\
& $\mathrm{R}$ & $3.00(2.71-3.32)$ & $2.70(2.51-2.90)$ & $4.83(3.46-6.73)$ \\
Current smoking to ever smoking & $\mathrm{H}, \mathrm{P}_{\mathrm{H}}$ & $10.61,<0.001$ & $5.61,<0.001$ & $9.95,<0.001$ \\
& $\mathrm{n}$ & 139 & 121 & 33 \\
& $\mathrm{~F}$ & $2.87(2.79-2.95)$ & $2.82(2.75-2.90)$ & $3.44(3.13-3.78)$ \\
& $\mathrm{R}$ & $3.46(3.07-3.90)$ & $3.27(3.02-3.54)$ & $5.05(3.51-7.25)$ \\
\hline
\end{tabular}

\footnotetext{
${ }^{a}$ Within each study, results are selected in the following order of preference, within each sex, for: smoking status-ever, current or current, ever according to analysis; unexposed group-never any product, never cigarettes, other; smoking product-any, cigarettes (ignoring other products), cigarettes only; overlapping studies-principal, subsidiary; and then for single sex results in preference to combined sex results. Results adjusted for the most potential confounders are selected.

${ }^{b} \mathrm{n}=$ number of estimates combined, $\mathrm{F}=$ fixed-effect meta-analysis $\mathrm{RR}(95 \% \mathrm{Cl}), \mathrm{R}=$ random-effects meta-analysis $\mathrm{RR}(95 \% \mathrm{Cl}), \mathrm{H}=$ heterogeneity chisquared per degree of freedom.
}

not appear inconsistent with those summarized in Table 11.

\section{F. Risk by age of starting to smoke}

There is rather limited evidence available for age of starting, with only 10 studies for COPD, three for $\mathrm{CB}$ and one for emphysema providing data usable in metaanalyses. Table 12 summarizes the meta-analysis results. Random-effects RRs for earliest compared to latest starting are significantly elevated for COPD (1.49, 1.26-1.76, $\mathrm{n}=14)$ and $C B(2.08,1.29-3.35, \mathrm{n}=6)$, but not for emphysema $(1.14,0.70-1.88, \mathrm{n}=2)$. The increase in risk with earlier starting seen for COPD is consistent with the results of the key value analyses, with randomeffects estimates rising to $3.12(2.07-4.70, \mathrm{n}=8)$ for categories containing 14, but not 18 years.

\section{G. Risk by pack-years}

Table 13 summarizes the results for pack-years, based on 24 studies for COPD, eight for CB and two for emphysema. Key value analysis was not attempted for emphysema, due to the limited data. For COPD and CB, a clear dose-response is seen, with meta-analysis RRs increased for categories containing 5, but not 20 packyears, and increasing monotonically with increasing pack-years. Random-effects estimates for categories containing 45, but not 20, pack-years are 3.69 (2.79-4.86, n = 15) for COPD, and 7.04 (5.06-9.79, $\mathrm{n}=36)$ for CB. The "highest vs. lowest" analyses confirm the existence of a dose-response relationship for all three outcomes, with random-effects estimates of $2.80(2.37-3.30, \mathrm{n}=31)$ for COPD, $3.09(2.33-4.10, \mathrm{n}=11)$ for $\mathrm{CB}$, and 2.42 (1.25-4.70) for emphysema. Fuller results are given in the Additional files. As for amount smoked, these results show that the dose-related increase can be clearly seen using alternative key values (1, 10, 20, 30, 999), and using least-adjusted rather than most-adjusted RRs. The additional file also summarizes results for quite a number of other studies presenting dose response data in a form that cannot readily be meta-analysed. Many of these reported a significantly increased risk with increasing pack-years.

\section{H. Risk by duration of smoking}

Evidence for duration of smoking that can be used in meta-analyses is only available for three studies for COPD, three for CB and two for emphysema. Table 14 summarizes the results of the meta-analyses, which for $\mathrm{CB}$ and emphysema are based on heterogeneous data. Random-effects RRs for longest compared to shortest duration show no clear pattern for COPD $(1.12,0.63$ $1.98, \mathrm{n}=3)$, CB $(2.25,0.68-7.42, \mathrm{n}=4)$, or emphysema (7.67, 0.15-390.65, $\mathrm{n}=2$ ).

\section{Risk by duration of quitting (vs. never smoking)}

The number of studies providing usable data for duration of quitting compared to never smoking is seven for COPD, and seven for $\mathrm{CB}$, but none for emphysema. As shown in Table 15, there is some evidence of higher risks in short-term quitters for COPD, with the shortest vs. longest random-effects meta-analysis estimate 2.21 (1.24$3.94, \mathrm{n}=10$ ) and a tendency for estimates to be lower for the longer-term quitters in the key value analysis, though the trend is not monotonic. For $\mathrm{CB}$, evidence of higher risks in short-term quitters is less convincing, with the shortest vs. longest estimate of $1.25(0.99-1.59, \mathrm{n}=11)$ not significant, and RRs varying little by key value. The results are limited by the variability of the categories used by different studies to classify duration of quitting. This makes it difficult to find a key scheme which includes sufficient numbers of studies across the range. For instance, for COPD, the key scheme shown in Table 15 includes only three RRs at the two shorter levels, whereas an alternative set of key values (20, 12 and 3 years, shown in the Additional files) incorporates only three RRs at the two longer levels. 


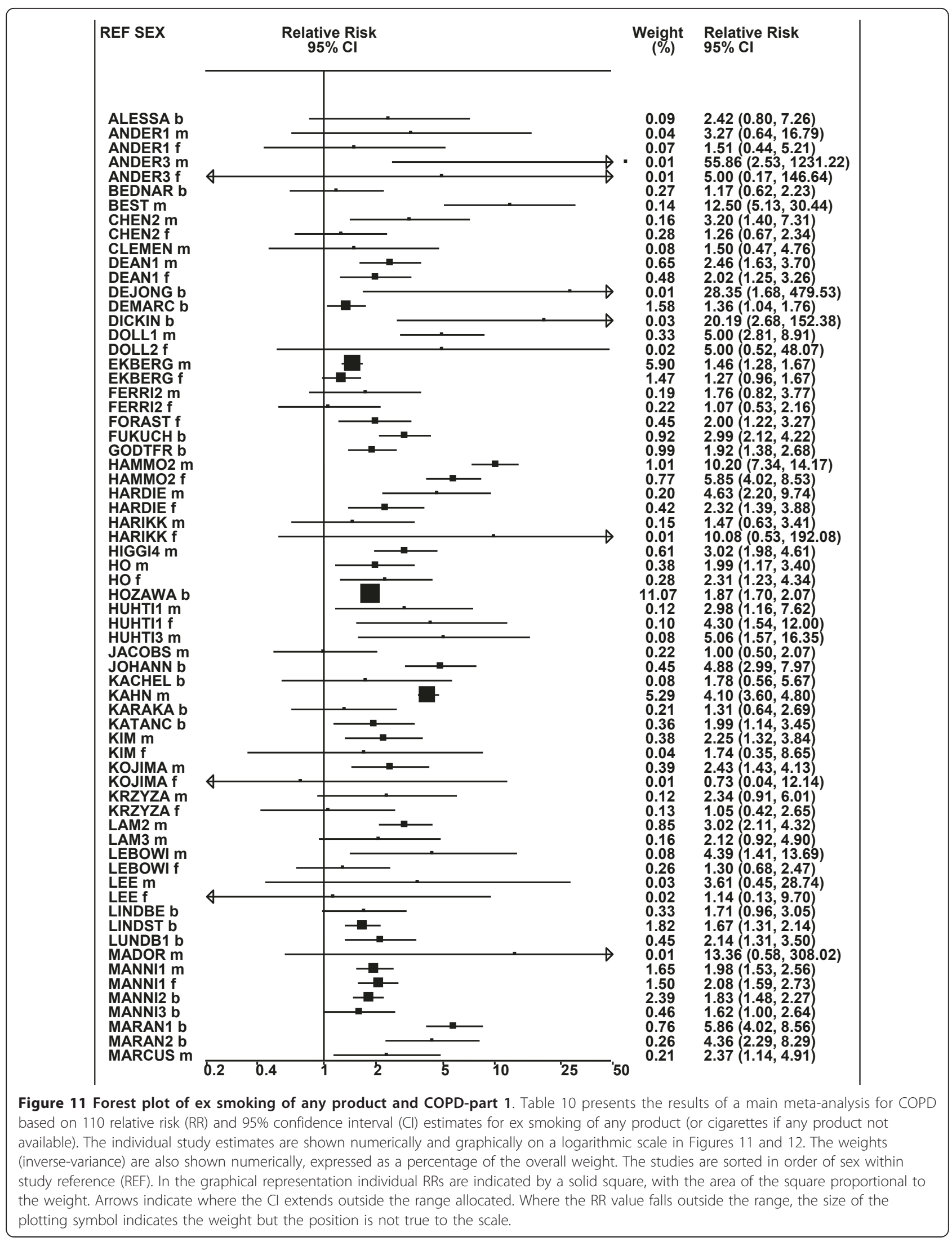




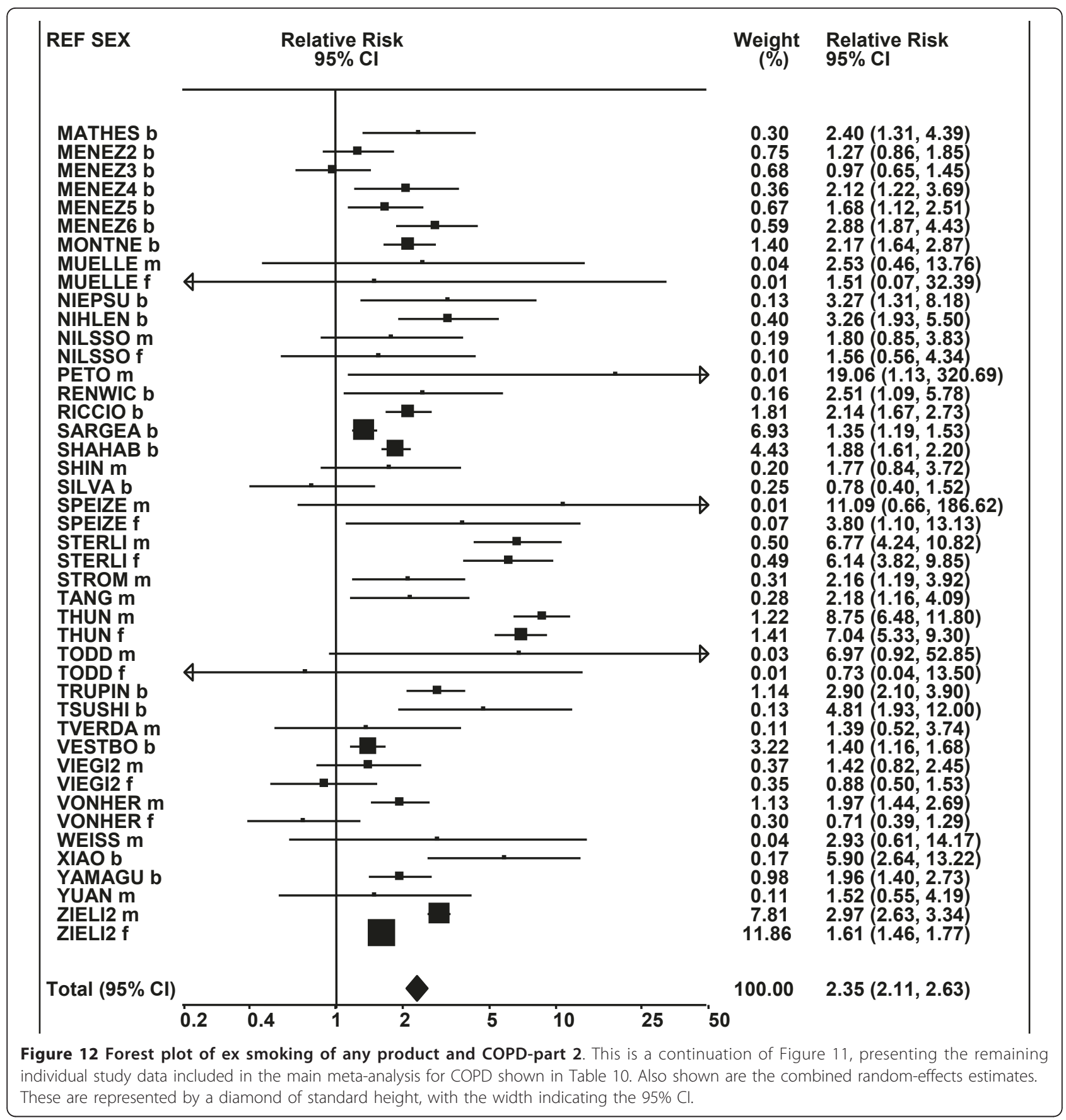

\section{J. Risk by duration of quitting (vs. current smoking)}

For duration of quitting compared to current smoking, data are available from one less study than for duration of quitting compared to never smoking for COPD, but from the same studies for $\mathrm{CB}$. The longest vs. shortest analysis shown in Table 16 is the inverse of the shortest vs. longest analysis in Table 15 . The key value analyses are based on a limited number of RRs but are consistent with the association declining with longer-term quitting. For categories including 12 , but not 7 , years quitting random-effects meta-analysis RRs relative to current smoking are $0.52(0.37-0.71, \mathrm{n}=9)$ for COPD and 0.65 (0.41-1.04, $\mathrm{n}=9)$ for CB.

Further analyses based on within-study differences

Some studies provide independent RRs for males and females for the same definition of outcome and exposure. Random-effects meta-analysis of the male/female sex ratio for current and ever smoking for each outcome confirm the impression already gained from the analyses 


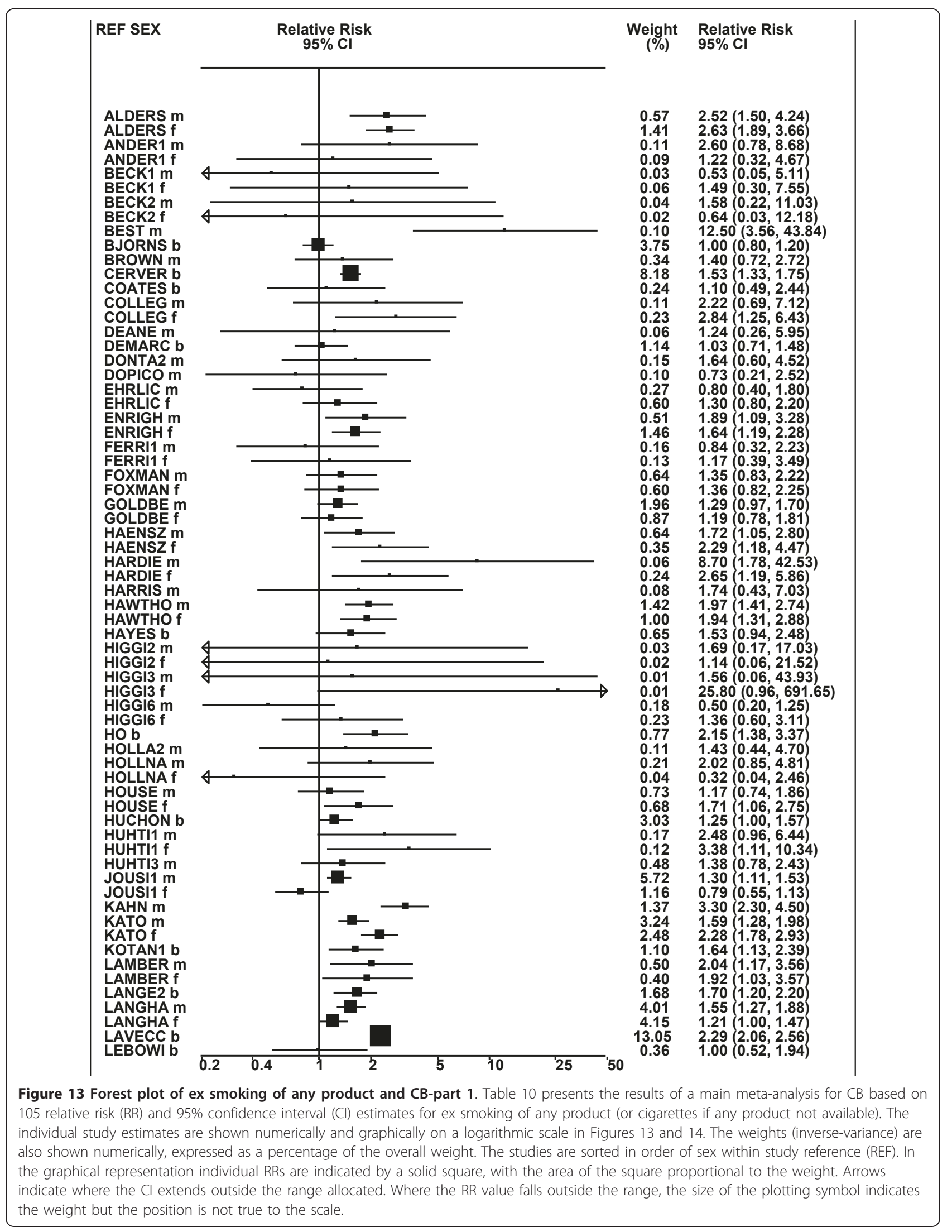




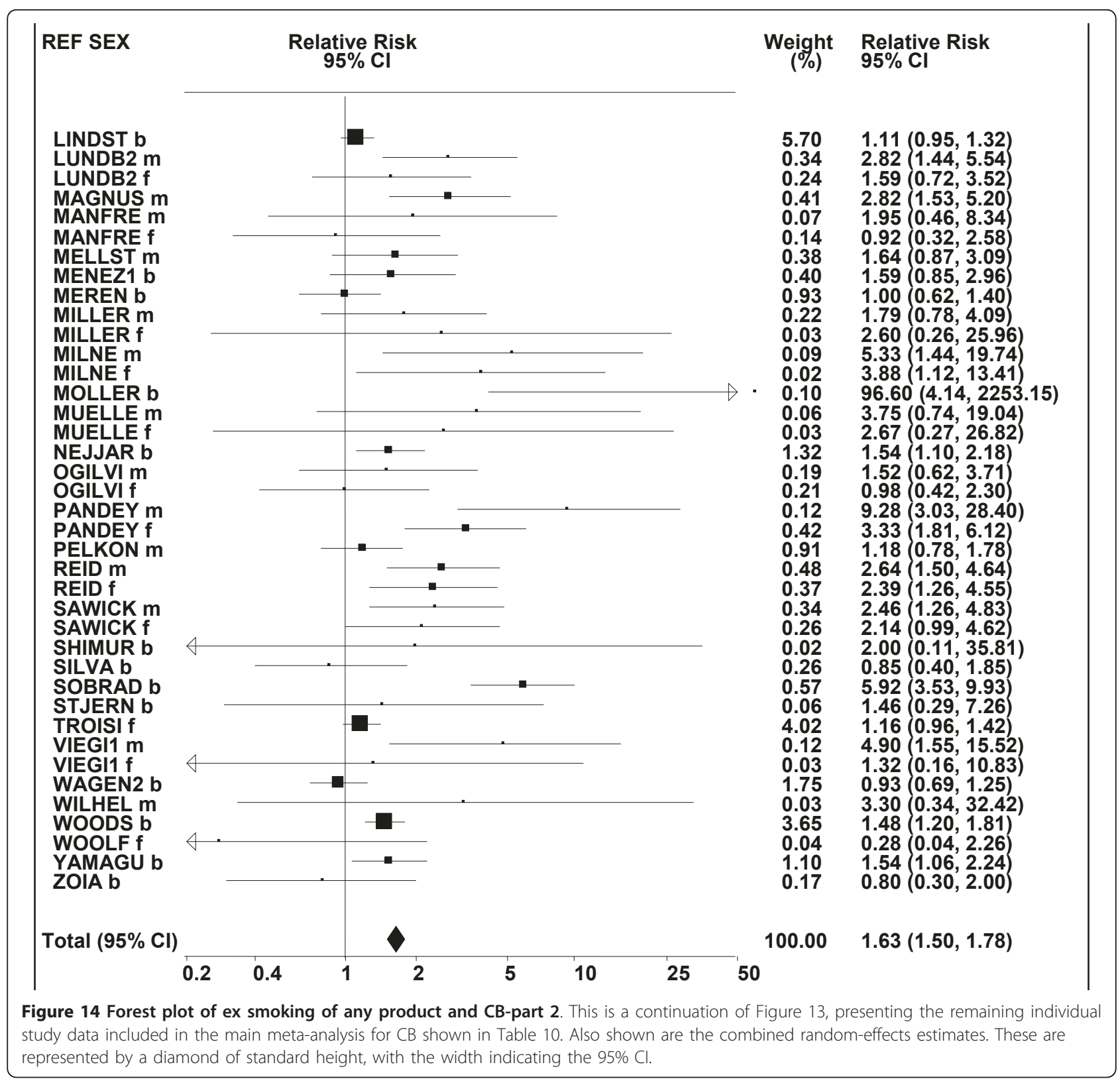

shown in Tables 5 to 8 that RRs are somewhat higher for males, though again the difference is not always statistically significant. For ever smoking, the meta-analysis RRs of the sex ratio are 1.28 (1.02-1.60) for COPD, 1.16 (0.97-1.38) for CB and 1.44 (0.72-2.87) for emphysema, based on, respectively, 31, 35 and 6 RRs. For current smoking the estimates are $1.25(1.00-1.58, \mathrm{n}=29)$ for COPD, $1.17(0.96-1.42, \mathrm{n}=35)$ for $\mathrm{CB}$, and $1.98(0.75-$ $5.22, \mathrm{n}=5$ ) for emphysema.

Some studies also provide separate non-independent least-adjusted and most-adjusted RRs for the same definition of exposure. There is little evidence that adjustment reduces the RR for ever or current smoking. For ever smoking, using the same preferences as in the main meta-analyses (Figures 1, 2, 3, 4 and 5), the mostadjusted estimate is lower than the least-adjusted estimate for 14 of the $30(46.7 \%)$ pairs for COPD, for 18 of the $41(43.9 \%)$ pairs for $\mathrm{CB}$, and for 2 of the $5(40.0 \%)$ pairs for emphysema. For current smoking the corresponding numbers are 11/26 (42.3\%) for COPD, 16/36 (44.4\%) for CB and $2 / 3$ (66.7\%) for emphysema. In no case do the percentages differ from $50 \%$ (at $\mathrm{p}<0.05$ ), and in each case the random-effects meta-analysis estimate based on the least-adjusted pair members is similar to the corresponding estimate based on the mostadjusted pair members (data not shown). 


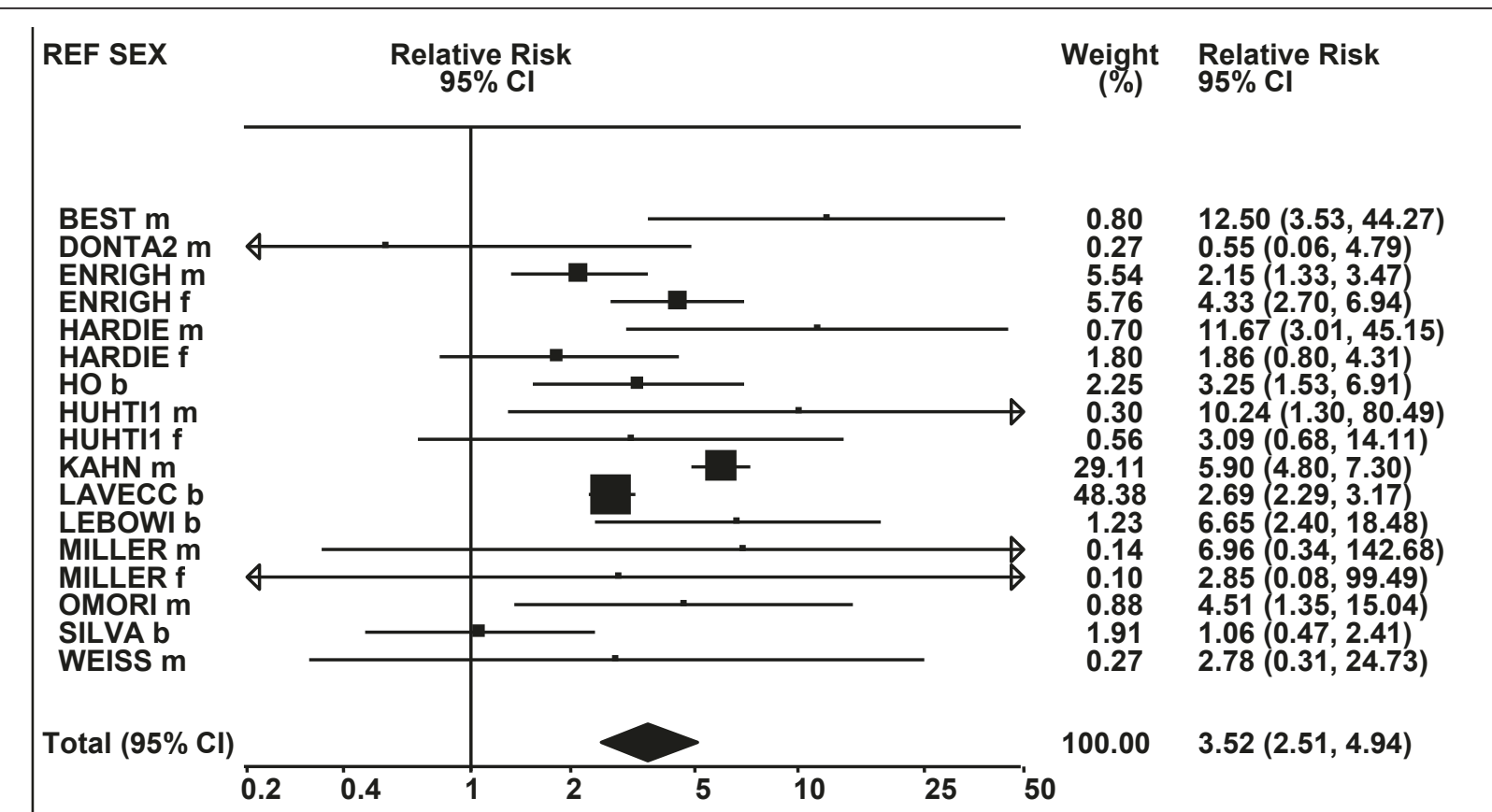

Figure 15 Forest plot of ex smoking of any product and emphysema. Table 10 presents the results of a main meta-analysis for emphysema based on 17 relative risk (RR) and 95\% confidence interval (Cl) estimates for ex smoking of any product (or cigarettes if any product not available). The individual study estimates are shown numerically and graphically on a logarithmic scale. The weights (inverse-variance) are also shown numerically, expressed as a percentage of the overall weight. The studies are sorted in order of sex within study reference (REF). In the graphical representation individual RRs are indicated by a solid square, with the area of the square proportional to the weight. Arrows indicate where the $\mathrm{Cl}$ extends outside the range allocated. Also shown are the combined random-effects estimates. These are represented by a diamond of standard height, with the width indicating the $95 \% \mathrm{Cl}$.

After excluding studies with no pipe or cigar smokers, some studies allow comparison of RRs of the risk of current smoking vs. never smoking for cigarette smokers ignoring other products with equivalent RRs for cigarette only smokers. These estimates are non-independent. For 7 of the 9 pairs of RRs for COPD, for all 6 of the pairs for $C B(p<0.05)$ and for both the pairs for emphysema the RR is lower for cigarette smokers ignoring other products. However the RR ratio is never markedly different from 1, ranging from 0.78 to 1.13 for COPD, from 0.84 to 0.99 for $\mathrm{CB}$, and from 0.86 to 0.96 for emphysema.

RRs for a dose-related index of smoking may be adjusted for other such indices. However, this is only at all common for age of starting to smoke, where adjustment for amount smoked is carried out in five of the 10 studies providing data for COPD, and in one of the three providing data for $\mathrm{CB}$. It is not possible to assess the effect of adjustment for amount smoked, as three of the six relevant studies provide the adjusted RR and no other RR, and the other three provide only adjusted and totally unadjusted RRs.

For all three outcomes, Egger's test [16] shows significant evidence of publication bias for both ever smoking (COPD $\mathrm{p}<0.001, \mathrm{CB} \mathrm{p}<0.05$, emphysema $\mathrm{p}<0.01$ ) and current smoking (COPD p $<0.05, \mathrm{CB} \mathrm{p}<0.001$, emphysema $\mathrm{p}<0.05)$. Figures 16 (COPD), 17 (CB) and 18 (emphysema) show funnel plots for ever smoking. All the plots give an impression of there being more lowerweight RRs above the mean and more higher-weight RRs below the mean.

\section{Discussion}

\section{Evidence of a relationship}

The meta-analyses carried out demonstrate a clear relationship of smoking to all three outcomes consideredCOPD, CB and emphysema. This is evident for ever, current and ex smoking, and for outcomes based on mortality, lung function, symptom prevalence or other methods. That this relationship is causal is supported by the evidence of a dose-response, risk increasing with amount smoked and pack-years for all three outcomes, and (based on more limited data) decreasing with increasing age of starting to smoke for COPD and CB, and with increasing duration of quitting for COPD. It is also supported by the similarity of results based on most-adjusted and least-adjusted RRs, and by withinstudy comparisons showing that additional confounder adjustment little affected estimates for the same exposure definition. 
Table 10 Main meta-analyses for ex smoking of any product (or cigarettes, if any product not available) ${ }^{a}$

\begin{tabular}{|c|c|c|c|c|c|}
\hline Factor & Level & Statistic $^{\mathrm{b}}$ & COPD & CB & Emphysema \\
\hline \multirow[t]{4}{*}{ All } & All & $\mathrm{N}$ & 110 & 105 & 17 \\
\hline & & $F$ & $2.12(2.05-2.19)$ & $1.56(1.50-1.62)$ & $3.50(3.13-3.92)$ \\
\hline & & $\mathrm{R}$ & $2.35(2.11-2.63)$ & $1.63(1.50-1.78)$ & $3.52(2.51-4.94)$ \\
\hline & & $\mathrm{H}, \mathrm{P}_{\mathrm{H}}$ & $7.43,<0.001$ & $3.14,<0.001$ & $3.87,<0.001$ \\
\hline \multirow[t]{10}{*}{ Sex } & Male & $n$ & 44 & 46 & 9 \\
\hline & & $\mathrm{F}$ & $2.80(2.64-2.97)$ & $1.63(1.52-1.76)$ & $5.12(4.26-6.15)$ \\
\hline & & $\mathrm{R}$ & $2.87(2.35-3.50)$ & $1.80(1.57-2.06)$ & $4.70(2.66-8.30)$ \\
\hline & Female & $n$ & 28 & 35 & 4 \\
\hline & & $\mathrm{F}$ & $1.92(1.78-2.06)$ & $1.52(1.40-1.65)$ & $3.50(2.36-5.19)$ \\
\hline & & $\mathrm{R}$ & $2.02(1.53-1.68)$ & $1.64(1.40-1.93)$ & $3.50(2.36-5.19)$ \\
\hline & Combined & $\mathrm{n}$ & 38 & 24 & 4 \\
\hline & & $\mathrm{F}$ & $1.83(1.74-1.92)$ & $1.54(1.46-1.63)$ & $2.68(2.30-3.12)$ \\
\hline & & $\mathrm{R}$ & $2.07(1.83-2.35)$ & $1.44(1.22-1.71)$ & $2.68(1.58-4.54)$ \\
\hline & Between levels & $P_{B}$ & $<0.001$ & NS & $<0.05$ \\
\hline \multirow[t]{13}{*}{ Continent } & N America & $n$ & 36 & 37 & 9 \\
\hline & & $F$ & $2.77(2.61-2.93)$ & $1.45(1.32-1.60)$ & $4.70(3.97-5.57)$ \\
\hline & & $\mathrm{R}$ & $2.98(2.35-3.78)$ & $1.47(1.25-1.73)$ & $3.82(2.30-6.34)$ \\
\hline & Europe & $n$ & 50 & 55 & 6 \\
\hline & & $\mathrm{F}$ & 1.81 (1.73-1.89) & $1.57(1.50-1.65)$ & $2.71(2.32-3.17)$ \\
\hline & & $\mathrm{R}$ & $1.99(1.76-2.25)$ & $1.71(1.51-1.94)$ & $3.00(1.70-5.28)$ \\
\hline & Asia & $n$ & 16 & 7 & 2 \\
\hline & & $\mathrm{F}$ & $2.84(2.91-3.25)$ & $1.94(1.69-2.23)$ & $3.56(1.88-6.75)$ \\
\hline & & $\mathrm{R}$ & $2.79(2.23-3.48)$ & $2.17(1.63-2.88)$ & $3.56(1.88-6.75)$ \\
\hline & Other or multicountry & $\mathrm{n}$ & 8 & 6 & 0 \\
\hline & & $F$ & $1.52(1.31-1.76)$ & $1.34(1.14-1.57)$ & \\
\hline & & $\mathrm{R}$ & $1.58(1.22-2.05)$ & $1.33(1.13-1.57)$ & \\
\hline & Between levels & $P_{B}$ & $<0.001$ & NS & $<0.05$ \\
\hline \multirow[t]{10}{*}{ Study type } & Case-control & $n$ & 11 & 9 & 0 \\
\hline & & $\mathrm{F}$ & $3.38(2.78-4.10)$ & $1.65(1.38-1.98)$ & \\
\hline & & $\mathrm{R}$ & $3.45(2.26-5.28)$ & $1.87(1.18-2.96)$ & \\
\hline & Prospective & $n$ & 35 & 10 & 4 \\
\hline & & $\mathrm{F}$ & $3.92(3.60-4.26)$ & $1.59(1.40-1.81)$ & $5.32(4.36-6.49)$ \\
\hline & & $\mathrm{R}$ & $2.88(2.23-3.73)$ & $1.79(1.24-2.60)$ & $2.99(0.91-9.84)$ \\
\hline & Cross-sectional & $n$ & 64 & 86 & 13 \\
\hline & & $\mathrm{F}$ & $1.85(1.78-1.92)$ & 1.55 (1.49-1.62) & $2.87(2.50-3.30)$ \\
\hline & & $\mathrm{R}$ & $1.99(1.81-2.19)$ & $1.60(1.45-1.75)$ & $3.16(2.50-4.00)$ \\
\hline & Between levels & $P_{B}$ & $<0.001$ & NS & $<0.01$ \\
\hline \multirow{10}{*}{$\begin{array}{l}\text { Outcome } \\
\text { subtype }\end{array}$} & Mortality & $n$ & 29 & 2 & 2 \\
\hline & & $\mathrm{F}$ & $4.41(4.04-4.80)$ & $3.61(2.61-4.99)$ & $6.02(4.90-7.40)$ \\
\hline & & $\mathrm{R}$ & $3.55(2.75-4.57)$ & $5.57(1.56-19.92)$ & $6.56(3.93-10.94)$ \\
\hline & $\begin{array}{l}\text { Lung function (COPD) or } \\
\text { symptoms (CB) }\end{array}$ & $n$ & 51 & 76 & - \\
\hline & & $\mathrm{F}$ & $1.86(1.78-1.93)$ & $1.39(1.32-1.46)$ & \\
\hline & & $\mathrm{R}$ & $1.92(1.72-2.13)$ & $1.50(1.36-1.64)$ & \\
\hline & Other & $\mathrm{n}$ & 30 & 27 & 15 \\
\hline & & $\mathrm{F}$ & $1.87(1.74-2.02)$ & $1.90(1.78-2.04)$ & $2.78(2.43-3.18)$ \\
\hline & & $\mathrm{R}$ & $2.18(1.80-2.64)$ & $1.84(1.58-2.14)$ & $2.95(2.23-3.91)$ \\
\hline & Between levels & $P_{B}$ & $<0.001$ & $<0.001$ & $<0.001$ \\
\hline \multirow[t]{2}{*}{$\begin{array}{l}\text { Smoking } \\
\text { product }\end{array}$} & Any & $\mathrm{n}$ & 43 & 53 & 4 \\
\hline & & $\mathrm{F}$ & $1.76(1.65-1.88)$ & $1.64(1.55-1.74)$ & $2.72(2.31-3.19)$ \\
\hline
\end{tabular}


Table 10 Main meta-analyses for ex smoking of any product (or cigarettes, if any product not available) ${ }^{\text {a }}$ (Continued)

\begin{tabular}{|c|c|c|c|c|c|}
\hline & & $\mathrm{R}$ & $2.09(1.78-2.45)$ & $1.67(1.45-1.93)$ & $2.72(2.31-3.19)$ \\
\hline & $\begin{array}{l}\text { Cigarettes (ignoring other } \\
\text { products) }\end{array}$ & $n$ & 59 & 49 & 12 \\
\hline & & $\mathrm{F}$ & 2.07 (1.99-2.15) & $1.46(1.38-1.54)$ & $4.42(3.76-5.18)$ \\
\hline & & $\mathrm{R}$ & $2.19(1.94-2.47)$ & $1.53(1.38-1.70)$ & $3.33(2.17-5.10)$ \\
\hline & Cigarettes only & $n$ & 8 & 3 & 1 \\
\hline & & $\mathrm{F}$ & $6.79(5.87-7.86)$ & $2.80(2.13-3.67)$ & $12.50(3.53-44.27)$ \\
\hline & & $\mathrm{R}$ & $5.78(3.81-8.77)$ & $3.28(1.84-5.84)$ & $12.50(3.53-44.27)$ \\
\hline & Between levels & $P_{B}$ & $<0.001$ & $<0.05$ & $<0.1$ \\
\hline \multirow{7}{*}{$\begin{array}{l}\text { Unexposed } \\
\text { base }^{c}\end{array}$} & Never any product & $n$ & 54 & 65 & 6 \\
\hline & & $\mathrm{F}$ & $2.41(2.28-2.54)$ & 1.69 (1.60-1.79) & $3.67(3.23-4.16)$ \\
\hline & & $\mathrm{R}$ & $2.60(2.12-3.17)$ & $1.75(1.53-2.01)$ & $4.70(2.58-8.53)$ \\
\hline & Never cigarettes & $\mathrm{n}$ & 56 & 40 & 11 \\
\hline & & $F$ & $1.96(1.88-2.04)$ & $1.44(1.36-1.52)$ & $2.93(2.28-3.76)$ \\
\hline & & $\mathrm{R}$ & $2.15(1.92-2.42)$ & $1.50(1.35-1.66)$ & $2.99(1.96-4.56)$ \\
\hline & Between levels & $P_{B}$ & $<0.05$ & $<0.05$ & NS \\
\hline
\end{tabular}

a Within each study, results are selected in the following order or preference, within each sex, for: unexposed group-never any product, never cigarettes, other; smoking product-any, cigarettes (ignoring other products), cigarettes only; overlapping studies-principal, subsidiary; and then for single sex results in preference to combined sex results. Results adjusted for the most potential confounders are selected.

${ }^{b} \mathrm{n}=$ number of estimates combined, $\mathrm{F}=$ fixed-effect meta-analysis $\mathrm{RR}(95 \% \mathrm{Cl}), \mathrm{R}=$ random-effects meta-analysis $\mathrm{RR}(95 \% \mathrm{Cl}), \mathrm{H}=\mathrm{heterogeneity} \mathrm{chisquared} \mathrm{per}$ degree of freedom, $P_{H}=$ probability value for heterogeneity expressed as $p<0.001, p<0.05, p<0.1$ or NS ( $p \geq 0.1$ ), $P_{B}=$ probability value for between levels (see methods) similarly expressed.

c Includes acceptable near-equivalent estimate (see methods) if estimate for strictly defined never smoker base not available (COPD: 3 for never cigarettes, CB: 2 for never any product and 4 for never cigarettes).

Table 11 Meta-analyses for amount smoked ${ }^{\mathrm{a}}$

\begin{tabular}{|c|c|c|c|c|}
\hline Amount smoked & Statistic $^{\mathbf{b}}$ & COPD & CB & Emphysema \\
\hline Number of sets ${ }^{c}$ & & 42 & 57 & 11 \\
\hline \multirow[t]{4}{*}{ About 5 cigs/day ${ }^{\text {d, g }}$} & $\mathrm{N}$ & 40 & 53 & 9 \\
\hline & $F$ & $2.58(2.39-2.78)$ & $2.04(1.91-2.16)$ & $2.21(1.88-2.59)$ \\
\hline & $\mathrm{R}$ & $2.89(2.41-3.45)$ & $2.32(2.07-2.60)$ & $4.24(1.88-9.55)$ \\
\hline & $\mathrm{H}, \mathrm{P}_{\mathrm{H}}$ & $3.58,<0.001$ & $2.08,<0.001$ & $7.82,<0.001$ \\
\hline \multirow[t]{4}{*}{ About 20 cigs/day ${ }^{e, ~ g}$} & $\mathrm{~N}$ & 23 & 33 & 6 \\
\hline & $\mathrm{F}$ & $6.24(5.79-6.73)$ & $3.64(3.33-3.98)$ & $7.10(4.83-10.44)$ \\
\hline & $\mathrm{R}$ & $6.21(4.72-8.17)$ & $4.43(3.68-5.32)$ & $5.07(2.04-12.61)$ \\
\hline & $\mathrm{H}, \mathrm{P}_{\mathrm{H}}$ & $7.83,<0.001$ & $3.32,<0.001$ & $3.56,<0.01$ \\
\hline \multirow[t]{4}{*}{ About 45 cigs/day f, g } & N & 26 & 35 & 6 \\
\hline & $\mathrm{F}$ & $9.83(8.85-10.92)$ & $6.00(5.48-6.57)$ & $12.39(7.49-20.50)$ \\
\hline & $\mathrm{R}$ & $9.50(7.38-12.22)$ & 7.37 (5.86-9.28) & 7.19 (2.74-18.87) \\
\hline & $\mathrm{H}, \mathrm{P}_{\mathrm{H}}$ & $3.28,<0.001$ & $4.66,<0.001$ & $2.23,<0.05$ \\
\hline \multirow[t]{4}{*}{ Highest v lowest } & N & 44 & 62 & 11 \\
\hline & $\mathrm{F}$ & $2.22(2.08-2.37)$ & $1.99(1.87-2.12)$ & $1.41(1.17-1.70)$ \\
\hline & $\mathrm{R}$ & $2.32(1.90-2.83)$ & $2.42(2.10-2.79)$ & $2.73(1.23-6.04)$ \\
\hline & $\mathrm{H}, \mathrm{P}_{\mathrm{H}}$ & $6.02,<0.001$ & $3.65,<0.001$ & $7.50,<0.001$ \\
\hline
\end{tabular}

${ }^{a}$ Within each study, results are selected in the following order of preference, within each sex, for: smoking status-current, ever; unexposed group (where relevant)-never any product, never cigarettes; smoking product-cigarettes (ignoring other products), cigarettes only, any product; overlapping studies-principal, subsidiary; and then for single sex results in preference to combined sex results. Results adjusted for the most potential confounders are selected.

${ }^{\mathrm{b}} \mathrm{n}=$ number of estimates combined, $\mathrm{F}=$ fixed-effect meta-analysis $\mathrm{RR}(95 \% \mathrm{Cl}), \mathrm{R}=$ random-effects meta-analysis $\mathrm{RR}(95 \% \mathrm{Cl}), \mathrm{H}=$ heterogeneity chisquared per degree of freedom, $\mathrm{P}_{\mathrm{H}}=$ probability value for heterogeneity expressed as $p<0.001, p<0.05, p<0.1$ or NS ( $p \geq 0.1$ ).

c Number of sets of RRs available for the key value analysis, where base for comparison is never smoked.

${ }^{\mathrm{d}}$ Category for which results are provided includes 5 cigs/day but does not include 20 cigs/day.

e Category for which results are provided includes 20 cigs/day but does not include 5 or 45 cigs/day.

${ }^{f}$ Category for which results are provided includes 45 cigs/day but does not include 20 cigs/day.

${ }^{g}$ Base for comparison is never smoked. 
Table 12 Meta-analyses for age started to smoke

\begin{tabular}{|c|c|c|c|c|}
\hline Age started & Statistic $^{\mathrm{b}}$ & COPD & $\mathrm{CB}$ & Emphysema \\
\hline Number of sets ${ }^{c}$ & & 10 & 2 & 2 \\
\hline \multirow[t]{4}{*}{ About age 26 years ${ }^{d, g}$} & N & 6 & Insufficient data & Insufficient data \\
\hline & $\mathrm{F}$ & $1.74(1.29-2.34)$ & & \\
\hline & $\mathrm{R}$ & $1.91(1.25-1.91)$ & & \\
\hline & $\mathrm{H}, \mathrm{P}_{\mathrm{H}}$ & $1.48, \mathrm{NS}$ & & \\
\hline \multirow[t]{4}{*}{ About age 18 years ${ }^{e, g}$} & N & 6 & Insufficient data & Insufficient data \\
\hline & $\mathrm{F}$ & $1.96(1.60-2.41)$ & & \\
\hline & $\mathrm{R}$ & $2.11(1.08-4.11)$ & & \\
\hline & $\mathrm{H}, \mathrm{P}_{\mathrm{H}}$ & $7.43,<0.001$ & & \\
\hline \multirow[t]{4}{*}{ About age 14 years $^{f, g}$} & $\mathrm{~N}$ & 8 & Insufficient data & Insufficient data \\
\hline & F & $3.34(2.74-2.08)$ & & \\
\hline & $\mathrm{R}$ & $3.12(2.07-4.70)$ & & \\
\hline & $\mathrm{H}, \mathrm{P}_{\mathrm{H}}$ & $2.88,<0.01$ & & \\
\hline \multirow[t]{4}{*}{ Earliest $\vee$ latest } & $\mathrm{N}$ & 14 & 6 & 2 \\
\hline & $\mathrm{F}$ & $1.41(1.30-1.52)$ & $1.99(1.42-2.79)$ & $1.14(0.70-1.88)$ \\
\hline & $\mathrm{R}$ & $1.49(1.26-1.76)$ & $2.08(1.29-3.35)$ & $1.14(0.70-1.88)$ \\
\hline & $\mathrm{H}, \mathrm{P}_{\mathrm{H}}$ & $1.29, \mathrm{NS}$ & $1.54, \mathrm{NS}$ & $0.01, \mathrm{NS}$ \\
\hline
\end{tabular}

${ }^{a}$ Within each study, results are selected in the following order of preference, within each sex, for: smoking status-current, ever; unexposed group (where relevant)-never any product, never cigarettes; smoking product-cigarettes (ignoring other products), cigarettes only, any product; overlapping studies-principal, subsidiary; and then for single sex results in preference to combined sex results. Results adjusted for the most potential confounders are selected.

${ }^{b} \mathrm{n}=$ number of estimates combined, $\mathrm{F}=$ fixed-effect meta-analysis $\mathrm{RR}(95 \% \mathrm{Cl}), \mathrm{R}=$ random-effects meta-analysis $\mathrm{RR}(95 \% \mathrm{Cl}), \mathrm{H}=\mathrm{heterogeneity} \mathrm{chisquared} \mathrm{per}$ degree of freedom, $\mathrm{P}_{\mathrm{H}}=$ probability value for heterogeneity expressed as $\mathrm{p}<0.001, \mathrm{p}<0.05, \mathrm{p}<0.1$ or NS ( $\mathrm{p} \geq 0.1$ ).

' Number of sets of RRs available for the key value analysis, where base for comparison is never smoked.

${ }^{\mathrm{d}}$ Category for which results are provided includes 26 years but does not include 18 years.

e Category for which results are provided includes 18 years but does not include 14 or 26 years.

${ }^{\mathrm{f}}$ Category for which results are provided includes 14 years but does not include 18 years.

${ }^{\mathrm{g}}$ Base for comparison is never smoked

\section{Heterogeneity}

The studies are remarkably consistent in reporting an increased risk in ever smokers. Only two of the $271 \mathrm{RRs}$ for the three outcomes combined considered in Figures 1, 2 and 3 are less than 1.0. However, studies also vary markedly in the magnitude of the estimated RR, as illustrated by the high values of $\mathrm{H}$ seen in the meta-analyses of the major smoking indices, which often exceed 5 and sometimes exceed 10 . ( $\mathrm{H}$ values of 5 and 10 are the same as $\mathrm{I}^{2}$ values [17] of $80 \%$ and $\left.90 \%\right)$. This is unsurprising given the many sources of variation involved, including sex, location, timing, study design and populations, definition of outcome and exposure, type of product smoked, and extent of confounder adjustment.

Using univariate and multivariate (meta-regression) methods, we investigated variation in risk by a number of characteristics of the study and the RR. For each outcome no characteristic on its own explains a major part of the variation, and substantial excess heterogeneity remains even after fitting multivariate models. However, differences in the strength of the association with smoking by level of some characteristics are apparent, these differences being quite similar for each outcome and each major smoking index. RRs tend to be higher for North American studies, for males, and for cigarette smoking than smoking of any product. For COPD RRs are substantially higher for studies of mortality or onset, especially those where the definition of COPD excludes asthma, and lower where the definition is lung function based. Studies of mortality are less common for $\mathrm{CB}$ or emphysema, but also give relatively high estimates. Effects of some other characteristics, such as study timing and study type, though significant in some univariate analyses, are not significant with the multivariate approach. As some characteristics are correlated (e.g. mortality studies are often prospective, US studies are more often prospective than elsewhere, and studies using lung function criteria are commonly cross-sectional) it is not straightforward to identify underlying effects. However, we feel that the main meta-regression models for ever smoking (Table 6) and current smoking (Table 8) for COPD and $\mathrm{CB}$ are useful in explaining some of the heterogeneity, their usefulness being confirmed by the fact that adding in further characteristics did not significantly improve prediction. Particularly for COPD, the metaregressions show there are many characteristics that independently modify the risk estimates. Meta-regressions were not tried for emphysema, where there were 
Table 13 Meta-analyses for pack-years ${ }^{a}$

\begin{tabular}{|c|c|c|c|c|}
\hline Pack-years & Statistic $^{\mathbf{b}}$ & COPD & $\mathrm{CB}$ & Emphysema \\
\hline $\begin{array}{l}\text { Number of } \\
\text { sets }^{c}\end{array}$ & & 28 & 11 & 3 \\
\hline \multirow[t]{4}{*}{ About $5^{\text {d, g }}$} & N & 23 & 10 & $\begin{array}{l}\text { Insufficient } \\
\text { data }\end{array}$ \\
\hline & $\mathrm{F}$ & $\begin{array}{l}1.13(1.06- \\
1.20)\end{array}$ & $\begin{array}{l}2.11(1.74- \\
2.55)\end{array}$ & \\
\hline & $\mathrm{R}$ & $\begin{array}{l}1.25(1.09- \\
1.44)\end{array}$ & $\begin{array}{l}1.74(1.17- \\
2.58)\end{array}$ & \\
\hline & $\mathrm{H}, \mathrm{P}_{\mathrm{H}}$ & $2.06,<0.01$ & $2.85,<0.01$ & \\
\hline \multirow[t]{4}{*}{ About $20^{\mathrm{e}, \mathrm{g}}$} & $N$ & 11 & 8 & $\begin{array}{l}\text { Insufficient } \\
\text { data }\end{array}$ \\
\hline & $F$ & $\begin{array}{l}1.68(1.58- \\
1.79)\end{array}$ & $\begin{array}{l}4.54(3.69- \\
5.58)\end{array}$ & \\
\hline & $\mathrm{R}$ & $\begin{array}{l}2.53(1.87- \\
3.43)\end{array}$ & $\begin{array}{l}4.54(3.69- \\
5.58)\end{array}$ & \\
\hline & $\mathrm{H}, \mathrm{P}_{\mathrm{H}}$ & $4.44,<0.001$ & $0.63, \mathrm{NS}$ & \\
\hline \multirow[t]{4}{*}{ About $45^{\text {e, }} \mathrm{g}$} & N & 15 & 8 & $\begin{array}{l}\text { Insufficient } \\
\text { data }\end{array}$ \\
\hline & $\mathrm{F}$ & $\begin{array}{l}3.14(2.97- \\
3.32)\end{array}$ & $\begin{array}{l}7.33(5.98- \\
8.97)\end{array}$ & \\
\hline & $\mathrm{R}$ & $\begin{array}{l}3.69(2.79- \\
4.86)\end{array}$ & $\begin{array}{l}7.04(5.06- \\
9.79)\end{array}$ & \\
\hline & $\mathrm{H}, \mathrm{P}_{\mathrm{H}}$ & $6.34,<0.001$ & $1.82,<0.1$ & \\
\hline \multirow{4}{*}{$\begin{array}{l}\text { Highest v } \\
\text { lowest }\end{array}$} & $\mathrm{N}$ & 31 & 11 & 3 \\
\hline & $\mathrm{F}$ & $\begin{array}{l}2.82(2.69- \\
2.97)\end{array}$ & $\begin{array}{l}2.52(2.25- \\
2.82)\end{array}$ & $\begin{array}{l}1.86(1.40- \\
2.47)\end{array}$ \\
\hline & $\mathrm{R}$ & $\begin{array}{l}2.80(2.37- \\
3.30)\end{array}$ & $\begin{array}{l}3.09(2.33- \\
4.10)\end{array}$ & $\begin{array}{l}2.42(1.25- \\
4.70)\end{array}$ \\
\hline & $\mathrm{H}, \mathrm{P}_{\mathrm{H}}$ & $4.09,<0.001$ & $2.23,<0.05$ & $1.79, \mathrm{NS}$ \\
\hline
\end{tabular}

a Within each study, results are selected in the following order of preference, within each sex, for: smoking status-current, ever; unexposed group (where relevant)-never any product, never cigarettes; smoking product-cigarettes (ignoring other products), cigarettes only, any product; overlapping studiesprincipal, subsidiary; and then for single sex results in preference to combined sex results. Results adjusted for the most potential confounders are selected.

${ }^{\mathrm{b}} \mathrm{n}=$ number of estimates combined, $\mathrm{F}=$ fixed-effect meta-analysis RR ( $95 \%$ $\mathrm{Cl}), \mathrm{R}=$ random-effects meta-analysis $\mathrm{RR}(95 \% \mathrm{Cl}), \mathrm{H}=$ heterogeneity chisquared per degree of freedom, $\mathrm{P}_{\mathrm{H}}=$ probability value for heterogeneity expressed as $p<0.001, p<0.05, p<0.1$ or NS ( $p \geq 0.1$ ).

c Number of sets of RRs available for the key value analysis, where base for comparison is never smoked.

${ }^{d}$ Category for which results are provided includes 5 pack-years but does not include 20 pack-years.

e Category for which results are provided includes 20 pack-years but does not include 5 or 45 pack-years.

${ }^{f}$ Category for which results are provided includes 45 pack-years but does not include 20 pack-years.

${ }^{\mathrm{g}}$ Base for comparison is never smoked.

fewer RRs available, or for ex smoking, where the relationship was weaker than for ever or current smoking. Sources of variation are discussed further in the following paragraphs.

\section{Sex}

If possible, sex-specific results are included in the metaanalyses, with combined sex results included only if not. Though variation by sex was not significant in all the
Table 14 Meta-analyses for duration of smoking ${ }^{a, b}$

\begin{tabular}{lllll}
\hline $\begin{array}{l}\text { Duration of } \\
\text { smoking }\end{array}$ & Statistic & COPD & CB & Emphysema \\
\hline $\begin{array}{l}\text { Longest vs. } \\
\text { shortest }\end{array}$ & $\mathrm{N}$ & 3 & 4 & 2 \\
& $\mathrm{~F}$ & $1.12(0.63-$ & $2.73(1.52-$ & $7.82(2.00-$ \\
& $\mathrm{1.98)}$ & $4.92)$ & $30.58)$ \\
& $\mathrm{R}$ & $1.12(0.63-$ & $2.25(0.68-$ & $7.67(0.15-$ \\
& & $1.98)$ & $7.42)$ & $390.65)$ \\
& $\mathrm{H}, \mathrm{P}_{\mathrm{H}}$ & $0.76, \mathrm{NS}$ & $3.60,<0.05$ & $8.31,<0.01$ \\
\hline
\end{tabular}

a Within each study, results are selected in the following order of preference, within each sex, for: smoking status-current, ever; never cigarettes; smoking product-cigarettes (ignoring other products), cigarettes only, any product; overlapping studies-principal, subsidiary; and then for single sex results in preference to combined sex results. Results adjusted for the most potential confounders are selected.

${ }^{\mathrm{b}}$ The number of sets of RRs available for key value analysis, where base for comparison is never smoked, is 3 for COPD, 4 for CB and 3 for emphysema, and no key value analysis was carried out.

${ }^{c} \mathrm{n}=$ number of estimates combined, $\mathrm{F}=$ fixed-effect meta-analysis RR (95\% $\mathrm{Cl}), \mathrm{R}=$ random-effects meta-analysis $\mathrm{RR}(95 \% \mathrm{Cl}), \mathrm{H}=$ heterogeneity chisquared per degree of freedom, $\mathrm{P}_{\mathrm{H}}=$ probability value for heterogeneity expressed as $p<0.001, p<0.05, p<0.1$ or NS ( $p \geq 0.1$ ).

main analyses, risk estimates generally tended to be higher for males than females. This is supported by additional analyses comparing RRs within-study for the same outcome and exposure definition. The higher RRs for males do not necessarily indicate any greater susceptibility, and seem more likely to reflect increased smoking. We note that some publications (e.g. [18-20]) have suggested that women may have a greater susceptibility than men to the effects of smoking on COPD or lung function, but others (e.g. [21-23]) have suggested the opposite. A detailed overall assessment of this aspect is beyond the scope of this paper.

\section{Age}

In the meta-regressions a continuous variable was included that indicated the midpoint of the age group to which the RR applied. The fitted coefficient was always positive, but significant (at $\mathrm{p}<0.05$ ) only for current smoking for COPD. Note that for each study only RRs for the whole age range were entered, though the availability of age-specific data was recorded. Proper assessment of the relationship of age to the RRs for the different outcomes would require entry and analysis of these further data. For the present, the data can only be regarded as indicating that $\mathrm{RRs}$ for studies in older populations may be greater than those in younger populations.

\section{Location}

The meta-regressions showed significant variation in risk by continent, mainly due to higher RRs for North American studies. Similar differences are seen in the univariate analyses for emphysema, and also for ex 
Table 15 Meta-analyses for duration of quitting (vs. never smoked) ${ }^{a}$

\begin{tabular}{llll}
\hline Duration of quitting & Statistic $^{\mathbf{b}}$ & COPD & CB \\
\hline Number of sets $^{\mathrm{c}}$ & & 10 & 11 \\
About 12 years $^{\mathrm{d} \text {, g }}$ & $\mathrm{n}$ & 10 & 9 \\
& $\mathrm{~F}$ & $3.45(2.96-4.01)$ & $1.40(1.21-2.62)$ \\
& $\mathrm{R}$ & $2.12(1.06-4.26)$ & $2.20(1.33-3.65)$ \\
& $\mathrm{H}, \mathrm{P}_{\mathrm{H}}$ & $12.74,<0.001$ & $8.04,<0.001$ \\
About 7 years $^{\mathrm{e}}$ g & $\mathrm{n}$ & 3 & 5 \\
& $\mathrm{~F}$ & $8.15(5.88-11.28)$ & $1.83(1.49-2.25)$ \\
& $\mathrm{R}$ & $4.94(1.21-20.07)$ & $2.36(1.29-4.32)$ \\
& $\mathrm{H}, \mathrm{P}_{\mathrm{H}}$ & $14.80,<0.001$ & $6.52,<0.001$ \\
About 3 years $^{\mathrm{f}}$ g & $\mathrm{n}$ & 3 & 7 \\
& $\mathrm{~F}$ & $3.58(2.44-5.25)$ & $2.16(1.82-2.57)$ \\
& $\mathrm{R}$ & $4.08(0.80-20.77)$ & $2.42(1.73-3.38)$ \\
& $\mathrm{H}, \mathrm{P}_{\mathrm{H}}$ & $16.66,<0.001$ & $2.79,<0.05$ \\
Shortest $v$ longest & $\mathrm{n}$ & 10 & 11 \\
& $\mathrm{~F}$ & $2.20(1.76-2.76)$ & $1.29(1.06-1.56)$ \\
& $\mathrm{R}$ & $2.21(1.24-3.94)$ & $1.25(0.99-1.59)$ \\
& $\mathrm{H}, \mathrm{P}_{\mathrm{H}}$ & $4.88,<0.001$ & $1.29, \mathrm{NS}$ \\
\hline
\end{tabular}

a Within each study, results are selected in the following order of preference, within each sex, for: smoking status-current, ever; unexposed group (where relevant)-never any product, never cigarettes; smoking product-cigarettes (ignoring other products), cigarettes only, any product; overlapping studiesprincipal, subsidiary; and then for single sex results in preference to combined sex results. Results adjusted for the most potential confounders are selected. ${ }^{\mathrm{b}} \mathrm{n}=$ number of estimates combined, $\mathrm{F}=$ fixed-effect meta-analysis RR $(95 \%$ $\mathrm{Cl}), \mathrm{R}=$ random-effects meta-analysis $\mathrm{RR}(95 \% \mathrm{Cl}), \mathrm{H}=$ heterogeneity chisquared per degree of freedom, $\mathrm{P}_{\mathrm{H}}=$ probability value for heterogeneity expressed as $p<0.001, p<0.05, p<0.1$ or NS $(p \geq 0.1)$.

' Number of sets of RRs available for the key value analysis, where base for comparison is never smoked. No data available for emphysema.

${ }^{\mathrm{d}}$ Category for which results are provided includes quit 12 years ago but does not include quit 7 years ago.

e Category for which results are provided includes quit 7 years ago but does not include quit 3 or 12 years ago.

${ }^{f}$ Category for which results are provided includes quit 3 years ago but does not include quit 7 years ago.

${ }^{9}$ Base for comparison is never smoked.

smoking (except for $\mathrm{CB}$ ). This difference is not readily explained, but it could relate to differences in diagnosis not fully accounted for by the model, in amount smoked, or in type of product smoked. However a variable accounting for the predominant long-term use of blended cigarettes in some countries (including the US), and of flue-cured Virginia cigarettes in others (including the UK and Canada), did not significantly predict risk.

\section{Study timing}

In the univariate analyses of ever and current smoking RRs varied significantly by when the study was published, but the pattern was erratic, with no trend. Study timing did not, however, add predictive power to the multivariate models. This suggests that differences between the periods studied are correlated with differences in other study characteristics. The term COPD has only been widely used in the last 25 years or so, and
Table 16 Meta-analyses for duration of quitting (vs. current smoking) ${ }^{a}$

\begin{tabular}{|c|c|c|c|}
\hline Duration of quitting & Statistic $^{\mathbf{b}}$ & COPD & $\mathrm{CB}$ \\
\hline Number of sets ${ }^{c}$ & & 9 & 11 \\
\hline \multirow[t]{4}{*}{ About 3 years $^{d, g}$} & $n$ & 2 & 7 \\
\hline & $\mathrm{F}$ & $0.77(0.51-1.15)$ & $1.07(0.91-1.25)$ \\
\hline & $\mathrm{R}$ & $0.77(0.51-1.15)$ & $1.00(0.70-1.43)$ \\
\hline & $\mathrm{H}, \mathrm{P}_{\mathrm{H}}$ & $0.28, \mathrm{NS}$ & $3.85,<0.001$ \\
\hline \multirow[t]{4}{*}{ About 7 years $^{\mathrm{e}, \mathrm{g}}$} & $\mathrm{n}$ & 2 & 5 \\
\hline & $\mathrm{F}$ & $1.03(0.62-1.70)$ & $1.00(0.84-1.19)$ \\
\hline & $\mathrm{R}$ & $1.03(0.62-1.70)$ & $0.87(0.47-1.61)$ \\
\hline & $\mathrm{H}, \mathrm{P}_{\mathrm{H}}$ & $0.53, \mathrm{NS}$ & $9.89,<0.001$ \\
\hline \multirow[t]{4}{*}{ About 12 years $^{\mathrm{e}, \mathrm{g}}$} & $\mathrm{n}$ & 9 & 9 \\
\hline & $\mathrm{F}$ & $0.52(0.43-0.63)$ & $0.61(0.53-0.69)$ \\
\hline & $\mathrm{R}$ & $0.52(0.37-0.71)$ & $0.65(0.41-1.04)$ \\
\hline & $\mathrm{H}, \mathrm{P}_{\mathrm{H}}$ & $1.93,<0.1$ & $9.11,<0.001$ \\
\hline \multirow[t]{4}{*}{ Longest $v$ shortest } & $\mathrm{n}$ & 10 & 11 \\
\hline & $\mathrm{F}$ & $0.43(0.34-0.53)$ & $0.78(0.64-0.95)$ \\
\hline & $\mathrm{R}$ & $0.45(0.24-0.84)$ & $0.80(0.63-1.02)$ \\
\hline & $\mathrm{H}, \mathrm{P}_{\mathrm{H}}$ & $5.78,<0.001$ & $1.28, \mathrm{NS}$ \\
\hline
\end{tabular}

a Within each study, results are selected in the following order of preference, within each sex, for: smoking product-cigarettes (ignoring other products), cigarettes only, any product; overlapping studies-principal, subsidiary; and then for single sex results in preference to combined sex results.

${ }^{\mathrm{b}} \mathrm{n}=$ number of estimates combined, $\mathrm{F}=$ fixed-effect meta-analysis RR $(95 \%$ $\mathrm{Cl}), \mathrm{R}=$ random-effects meta-analysis $\mathrm{RR}(95 \% \mathrm{Cl}), \mathrm{H}=$ heterogeneity chisquared per degree of freedom, $\mathrm{P}_{\mathrm{H}}=$ probability value for heterogeneity expressed as $p<0.001, p<0.05, p<0.1$ or NS ( $p \geq 0.1$ ).

c Number of sets of RRs available for the key value analysis, where base for comparison is current smoking. No data available for emphysema.

${ }^{\mathrm{d}}$ Category for which results are provided includes quit 3 years ago but does not include quit 7 years ago.

${ }^{\mathrm{e}}$ Category for which results are provided includes quit 7 years ago but does not include quit 3 or 12 years ago.

${ }^{f}$ Category for which results are provided includes quit 12 years ago but does not include quit 7 years ago.

${ }^{g}$ Base for comparison is current smoking.

definitions based on lung-function have been changing, so there may well be differences by time in the nature of outcomes we classified as COPD. There have also been changes in the nature of the product smoked, with reducing tar deliveries of cigarettes and declining use of pipes and cigars[24].

\section{Definition of the disease outcome}

For all RRs meta-analysed, the outcome had to be $\mathrm{CB}$ specifically, emphysema specifically or COPD generally. Thus each RR applied only to one outcome. The term COPD is quite recent, so data from some earlier studies which might legitimately have been included may have been excluded or entered against the wrong outcome. Some early studies described their outcomes as CB. If they supported their definitions by ICD codes incorporating all the core components of COPD, we reclassified the outcome as COPD. However, where ICD codes were not given, we left the outcome as $\mathrm{CB}$, though we suspect 


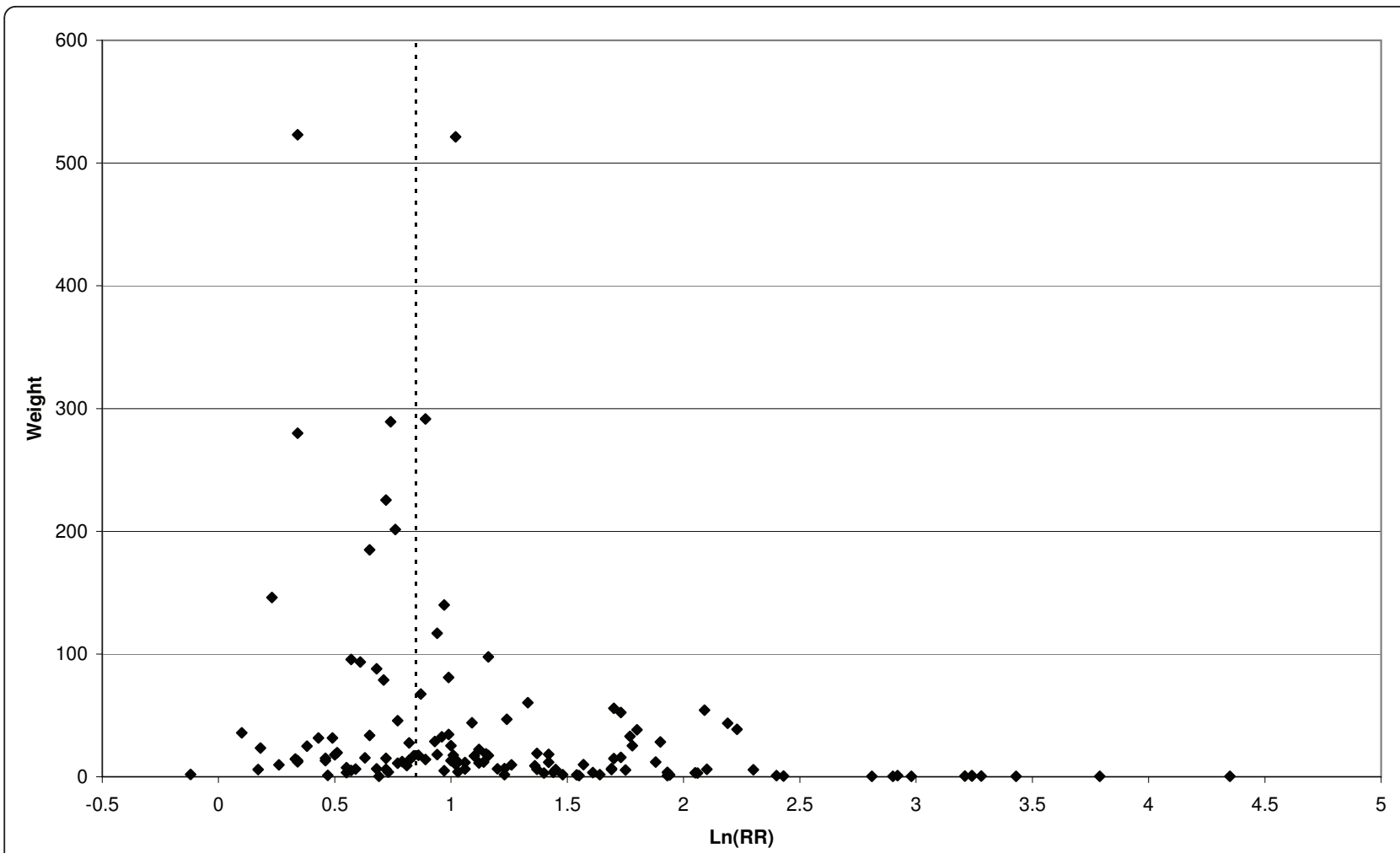

Figure 16 Funnel plot for ever smoking and COPD. Funnel plot of the 129 relative risk estimates for ever smoking and COPD included in the main meta-analysis in Table 5 against their weight (inverse-variance of log RR). The dotted vertical line indicates the fixed-effect meta-analysis estimate.

that sometimes the outcome might better have been COPD.

For COPD, the definitions allowed vary considerably, and the cases may not represent a homogeneous set. Thus population-based cross-sectional studies using lung function criteria alone probably include cases with less severe disease than studies in hospitals or using mortality records. Most prospective studies of incidence made no attempt to trace deaths, so may have omitted more rapidly progressive cases. We have not studied variation in risk in those few studies presenting results by severity of disease. Similar considerations apply to $\mathrm{CB}$ and emphysema, though less strongly, partly because there were fewer studies of mortality.

For COPD RRs are higher when the definition was based on mortality than when based on lung function or other criteria. Compared to RRs based on lung function, the meta-regressions indicated that RRs based on mortality are about 1.5 times higher for both ever and current smoking. The tendency for RRs based on mortality to be higher is also seen for $\mathrm{CB}$ and emphysema, but based on fewer studies.

For COPD RRs also clearly vary by how asthma was taken into account. For most studies, co-existing asthma was ignored (i.e. diagnosis was made independent of asthma, and both cases and non-cases could include asthmatics). However there were some (mainly mortality) studies where asthma is part of the outcome definition (e.g. COPD $=\mathrm{CB}$, emphysema or asthma). Here, usually only the underlying cause of death is considered, so the possibility of a CB or emphysema case also being recorded as having asthma does not arise. RRs are much lower for these studies. For others, asthmatics had been totally excluded, and RRs tend to be intermediate.

\section{Study type}

For COPD particularly, the univariate analyses show a tendency for RRs to be higher for prospective studies than for other designs. Study type did not contribute in multivariate analyses, probably reflecting its strong correlation with disease outcome definition, prospective studies tending to present mortality results, but other study types tending to use lung function, symptoms or other criteria.

\section{Aspects of smoking}

For COPD the meta-regressions show significant variation by smoking product, with RRs highest for smokers of cigarettes only, lowest for smokers of any product, 


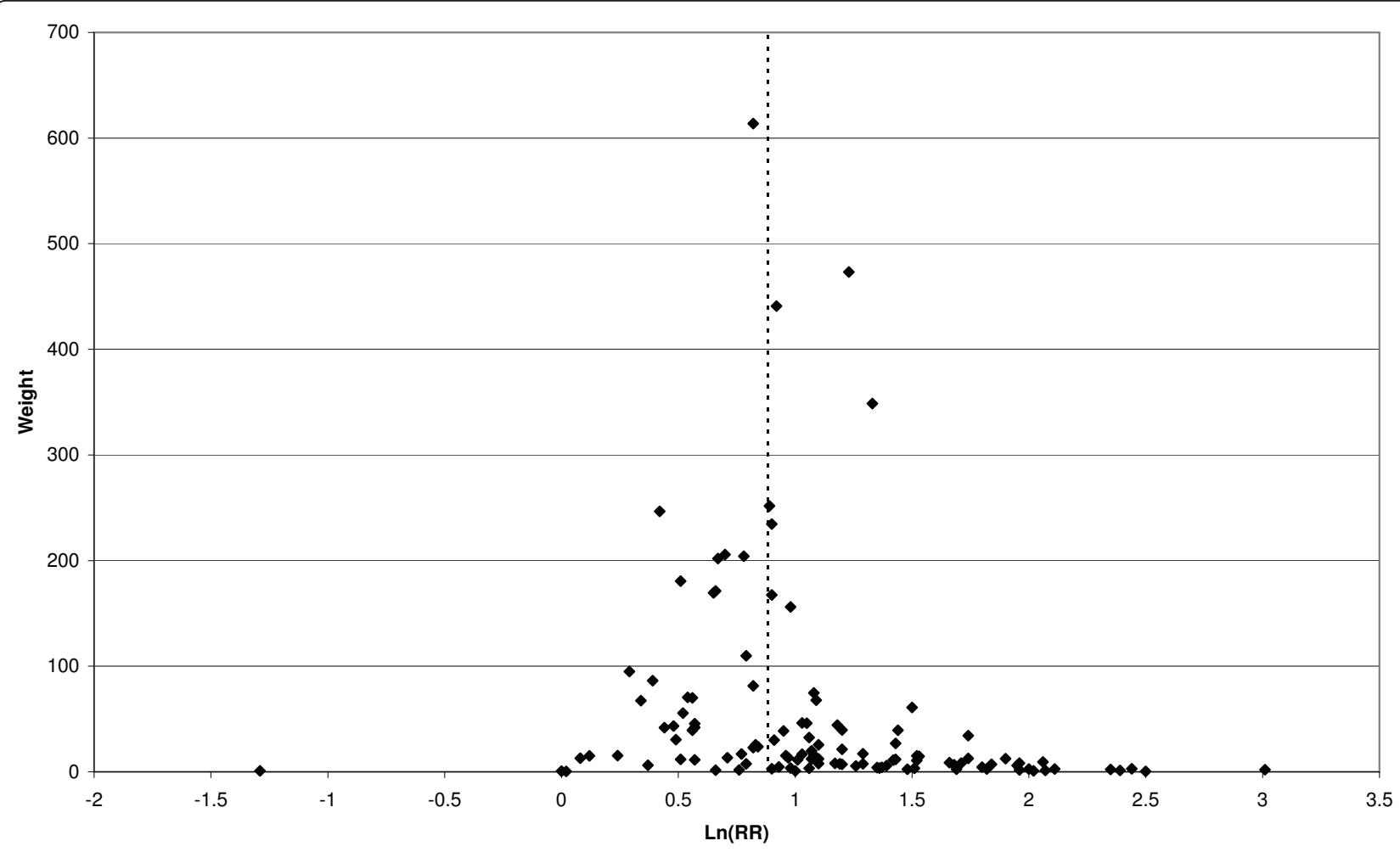

Figure 17 Funnel plot for ever smoking and CB. Funnel plot of the 114 relative risk estimates for ever smoking and CB included in the main meta-analysis in Table 5 against their weight (inverse-variance of log RR). The dotted vertical line indicates the fixed-effect meta-analysis estimate.

and intermediate for smokers of cigarettes (ignoring other products). As the estimates for cigarette only smokers depended largely on just two large studies (HAMMO2, THUN), we further investigated the difference between smokers of cigarettes only and smokers of cigarettes by within-study comparisons. This confirmed the tendency for cigarette only smokers to have higher risks. Though we have not considered data for smoking of pipes and cigars only, the results are consistent with a greater effect of smoking cigarettes than other products. Smokers of any product include some who smoke no cigarettes at all, while smokers of cigarettes include some who smoke cigarettes and pipes/cigars and who are likely to smoke less cigarettes per day than smokers of cigarettes only. For $\mathrm{CB}$ and emphysema there are few RRs for cigarette only smokers, but these also suggest higher risks for this group.

For COPD, the results show a higher RR where the unexposed group is never any product than when it is never cigarettes. This is consistent with the absolute risk being higher where the unexposed group includes some smokers (of pipes/cigars), than where it does not. However, this pattern is not seen for $\mathrm{CB}$ and emphysema.

We investigated the dose-response relationship by meta-analyses for five exposure measures-amount smoked, age of starting, pack-years, duration of smoking, duration of quitting (both vs. never smokers and vs. current smokers).

Meta-analysis of RRs expressed relative to never smokers or relative to current smokers is hampered by the different categories used by different studies to define level of exposure, so we also analyzed RRs comparing extreme levels of exposure within smokers, an approach allowing all studies to be included (including those only presenting analyses for smokers). For all three outcomes, risk increases with amount smoked and pack-years. For COPD and CB earlier starters have significantly higher risks, and risk also tended to decrease with longer-term quitting. Data are too few for emphysema to make inferences for age of starting and duration of quitting. The only measure showing no dose-relationship is duration of smoking but data are very limited. Note that all the outcomes are chronic diseases and disease presence may affect smoking habits. Depending on when smoking habits are recorded, this may bias downwards associations with these dose-related measures.

\section{Derivation of RRs}

About a third of RRs used in meta-analyses are available from the source or can be derived directly from crosstables of exposure by outcome. Otherwise more complex methods had to be used to derive the required RR. 


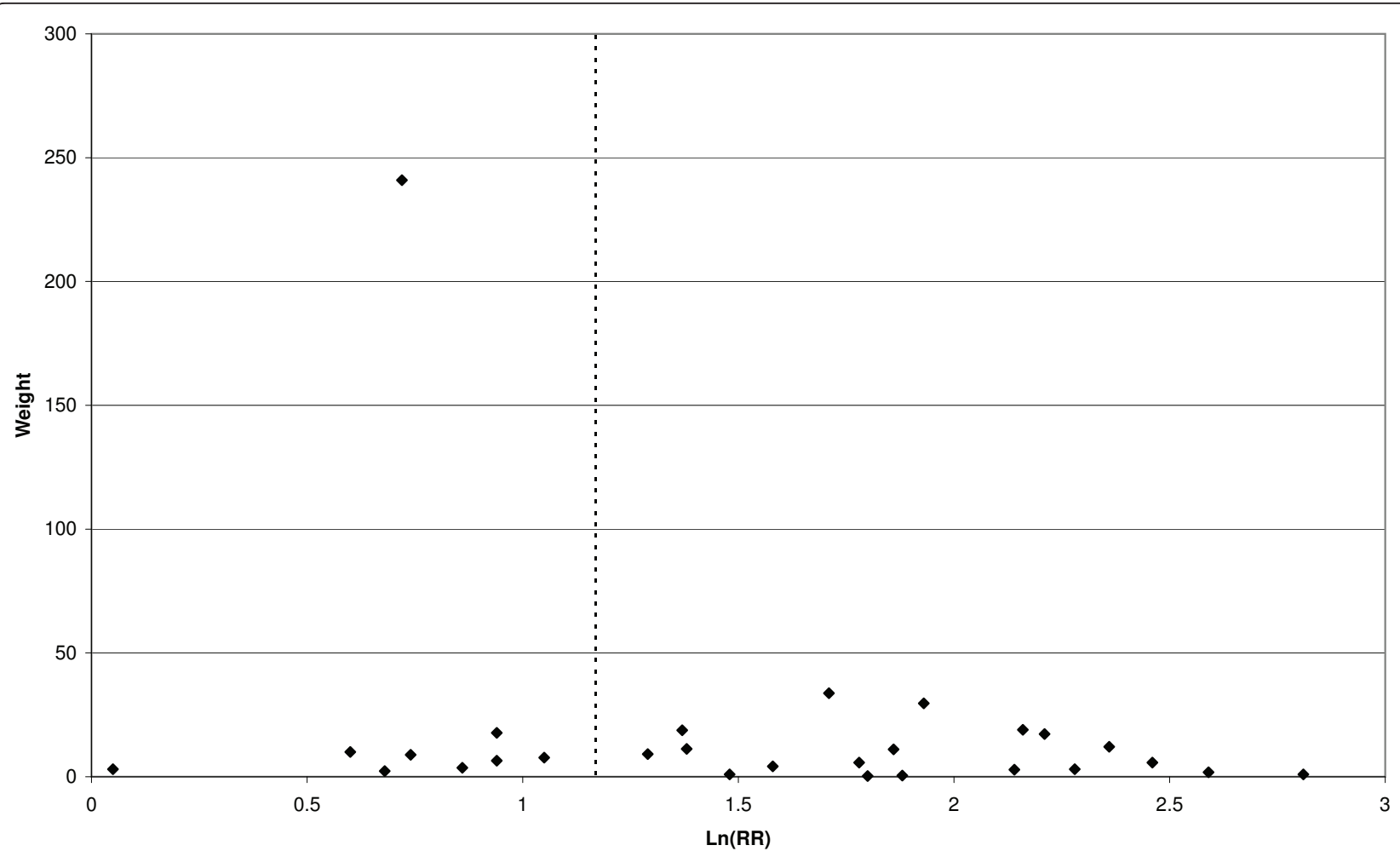

Figure 18 Funnel plot for ever smoking and emphysema. Funnel plot of the 28 relative risk estimates for ever smoking and emphysema included in the main meta-analysis in Table 5 against their weight (inverse-variance of log RR). The dotted vertical line indicates the fixed-effect meta-analysis estimate.

It was reassuring that whether or not the $R R$ was derived did not add predictive power to the main metaregression model, suggesting that use of derived RRs caused no material bias.

\section{Effect of studies with high RRs or large weight}

The statistical analyses investigated the role of various characteristics on the estimated risk of the three outcomes in relation to smoking, but did not formally test the effect of exclusion of specific studies with extreme RRs or large weights. For ever and current smoking, we have noted the highest RRs and those contributing most to the total weight. For COPD and CB, where each analysis involves over 100 most-adjusted RRs, no single RR contributes more than $12 \%$ of the total weight, and the distribution of RRs and of standardized residuals from the meta-regression models did not suggest any single RR had an undue influence. For emphysema, the situation is different. There are fewer RRs, only 28 for ever smoking and 22 for current smoking, and one study (LAVECC) contributes substantially to the overall weight (49\% for ever, $62 \%$ for current) while having a relatively low RR (2.05 for ever, 1.76 for current). Furthermore, study AUERBA, which does not provide an RR for ever smoking, has a strikingly large RR of 489.54 for current smoking.

We therefore investigated the effect of exclusion of these studies on the combined current smoking RR, where the problem is most severe (Table 17). It can be seen that exclusion of AUERBA substantially reduces the random-effects estimate, while exclusion of LAVECC substantially increases the fixed-effects estimate. Both exclusions, particularly AUERBA, reduce the heterogeneity substantially.

Why should the estimates vary so much? LAVECC was a large national health survey in Italy, in which 437/22, $376(2.0 \%)$ male and female current smokers of any product and 595/44, $172(1.3 \%)$ male and female never smokers of any product reported they had emphysema or respiratory insufficiency, with no independent check on the diagnosis. AUERBA involved an examination of whole-lung sections prepared from lungs removed at autopsy, with 816/839 (97.3\%) male current cigarette smokers and 20/176 (11.4\%) male never smokers of any product diagnosed as having minimal, slight, moderate, advanced or far advanced emphysema. These percentages differ widely between the two studies and reflect differences in 
Table 17 Investigating the effect of excluding a study with a very large weight, (LAVECC) and/or a study with a very high RR (AUERBA) on the meta-analysis, estimate for current smoking for emphysema

\begin{tabular}{lccc}
\hline Studies included & Fixed-effects RR (95\% CI) & Random-effects RR (95\% CI) & $\mathbf{H}^{\mathbf{a}}$ \\
\hline All 22 studies & $2.61(2.33-2.93)$ & $4.87(2.83-8.41)$ & 11.54 \\
Exclude AUERBA & $2.36(2.10-2.65)$ & $3.62(2.50-5.24)$ & 4.48 \\
Exclude LAVECC & $4.95(4.10-5.97)$ & $5.20(2.85-9.49)$ & 8.50 \\
Exclude both & $3.89(3.21-4.71)$ & $3.85(2.71-5.47)$ & 2.55 \\
\hline
\end{tabular}

${ }^{\mathrm{a}} \mathrm{H}$ is the heterogeneity chisquared per degree of freedom.

what is considered emphysema. Someone interviewed in a survey would be unaware of lower grades of emphysema. For AUERBA it is possible to derive RRs for higher grades of emphysema. For instance, restricting attention to advanced or far advanced emphysema reduces the rate in the male smokers to $134 / 839$ $(16.0 \%)$, and in never smokers to zero, so still indicating an extremely high RR.

We also compared the results reported by AUERBA with those reported in the other autopsy studies (ANDER2, PRATT, RYDER and SUTINE), although only results for ever smoking are available in those studies, PRATT being of males and the other studies of both sexes combined. Among never smokers of any product, rates of emphysema (ANDER2 30/51 = 58.8\%, PRATT $15 / 97=15.5 \%$, RYDER $21 / 73=28.8 \%$, SUTINE $28 / 73=38.4 \%$ ) are all much higher than reported by LAVECC and also higher than reported by AUERBA. Among ever smokers of any product (cigarettes only for ANDER2), rates of emphysema (ANDER2 $=89.5 \%$, PRATT $=42.0 \%$, RYDER $=75.5 \%$, SUTINE $=69.2 \%)$ are again much higher than reported by LAVECC but clearly lower than reported by AUERBA. While it is clear that emphysema rates based on autopsy studies are much higher than those based on surveys, (and also than those based on mortality studies, data not shown), the very high RR seen in AUERBA is due to a far greater discrimination between smokers and never smokers than seen in other autopsy studies. These results emphasise the problem of heterogeneity in deriving combined estimates.

\section{Representativeness}

We excluded studies of populations with a co-existing medical condition, with clearly atypical smoking habits (e.g. cocaine users or residents of a homeless shelter), or with clearly atypical risk (e.g. alpha-1 antitrypsin deficiency). Thus most studies include subjects broadly representative of the general population. Some studies had eligibility criteria such as long-term residence, household residence (excluding residents of institutions or military personnel) or telephone subscribers, criteria that may have resulted in underrepresenting subjects with lower SES or more mobile lifestyles. A few studies involved patients attending their physician or clinics, who may have been less healthy than average. It seems unlikely that any of these effects would have materially affected the relationship between smoking and COPD.

Studies of subjects with a high occupational risk for respiratory disease were excluded. The classification of high risk was based on our educated judgment, and not formally tested. Low occupational risk studies included in this report involved armed forces personnel, doctors, nurses, teachers, civil servants, professional and businessmen, coffeehouse and shop workers, postal, telephone, transport and clerical workers, and outdoor workers, as well as persons working in specific factories, research facilities, or unspecified industry.

Some studies included were originally designed along clinical or experimental rather than epidemiological lines, and subject selection was unclear. These studies are generally small, and any non-representativeness would little affect our results.

\section{Other sources of bias}

It is well known that researchers are more likely to wish to publish, and editors more likely to accept for publication, studies finding a statistically significant association between exposure and disease. The published literature may therefore overstate any true association or produce a false-positive relationship. There is some formal evidence of publication bias, with Egger's test suggesting bias in a number of the meta-analyses (see Figures 10 to 12). While some small studies showing no association may never have been published, large studies are likely to publish, and it is these which contribute most to the meta-analyses. We have not attempted to quantify bias, as formal methods are all based on assumptions which cannot be tested, but it seems doubtful whether publication bias is a serious issue.

Another possible source of bias is misclassification of smoking status. Random misclassification would dilute the association, as would any tendency for cases to deny or understate their smoking more than for the general population. Any tendency for current smokers to claim to be ex smokers, as might happen in a study conducted in a clinical setting or where patients have been advised to stop 
smoking, would tend to inflate the risk for ex smoking. Not only may misclassification rates vary by aspects of the study design and the way questions are asked, they may also vary by sex, age or other demographic variables.

The meta-analyses were conducted by combining direct estimates of the RR (from prospective studies) with ORs (from case-control and cross-sectional studies and occasionally from prospective studies). ORs somewhat overestimate relative risks where the disease is not rare [25], but here the overestimation is of little practical importance. Based on unadjusted data from prospective studies, where one could calculate both the relative risk and the OR, we estimate that the median bias from using the OR would have been only 1.01 for COPD and emphysema, and 1.04 for chronic bronchitis.

\section{Limitations}

This review has various limitations, many unavoidable. Lack of access to individual subject data limits the ability to carry out meta-analyses using similar exposure indices and confounder adjustment throughout, but obtaining such data was not feasible given many studies were conducted years ago. Obtaining a reliable definition of outcome and exposure is often hindered by incomplete information in the source papers. This review is also to some extent limited by restricting attention only to stratification by sex, and not attempting to record RRs subdivided by age or other characteristics. We also limited attention to specific indices of smoking, for example not entering data on pipe or cigar smoking, filter/plain smoking, or tar level. However we have recorded the availability of such extra information, and further work incorporating such data may give more insights. The procedures conducted for this review were extremely timeconsuming and it was impractical to bring the literature included fully up-to-date. However consideration of data from 218 studies published between 1953 and 2006 should give a reliable enough picture.

\section{Conclusions}

After excluding studies conducted in children or adolescents, or in populations at high respiratory disease risk or with co-existing diseases, we identified, from papers published between 1953 and 2006, 218 studies which relate one or more of a defined set of smoking indices to COPD, CB and emphysema. One hundred and thirtythree of the studies provide relevant data for COPD, 101 for $\mathrm{CB}$ and 28 for emphysema.

One major conclusion is that for each outcome the RRs for a given smoking index were markedly heterogeneous.

Another conclusion is that estimates are clearly elevated for all three outcomes. Individual study RRs virtually all exceed 1.0, and based on random-effects metaanalyses of most-adjusted RRs, estimates are elevated for ever smoking (COPD 2.89, CI 2.63-3.17, $\mathrm{n}=129$ RRs; CB 2.69, 2.50-2.90, $\mathrm{n}=114$; emphysema 4.51, 3.38-6.02, $\mathrm{n}=28$ ), current smoking (COPD 3.51, 3.08-3.99, $\mathrm{n}=$ 120; CB 3.41, 3.13-3.72, $\mathrm{n}=113$; emphysema 4.87, 2.838.41, $\mathrm{n}=22$ ) and ex smoking (COPD 2.35, 2.11-2.63, $\mathrm{n}$ $=110$; $\mathrm{CB} 1.63,1.50-1.78, \mathrm{n}=105)$; emphysema 3.52 , 2.51-4.94, $\mathrm{n}=17$ ). The consistency and strength of the relationships are consistent with a causal relationship. A causal relationship is supported by the fact that estimates are not materially affected by adjustment for confounding variables, and by the evidence of a doseresponse relationship, with risk increasing with amount smoked and pack-years for all three outcomes and (based on more limited data) risk decreasing with increasing starting age for $\mathrm{COPD}$ and $\mathrm{CB}$ and with increasing quitting duration for COPD.

Our review also provides evidence that various characteristics of the study and RR affect risk estimates. For COPD, RRs are higher for males, for studies conducted in North America, for cigarette smoking rather than any product smoking, where the unexposed base is never smoking any product, and are markedly lower when asthma is included in the COPD definition. Variations by sex, continent, smoking product and unexposed group are in the same direction for $\mathrm{CB}$, but less clearly demonstrated. For all outcomes RRs are higher when based on mortality, and for COPD are markedly lower when based on lung function.

This comprehensive review provides further insight into the relationship of smoking to COPD, $\mathrm{CB}$ and emphysema.

\section{Additional material}

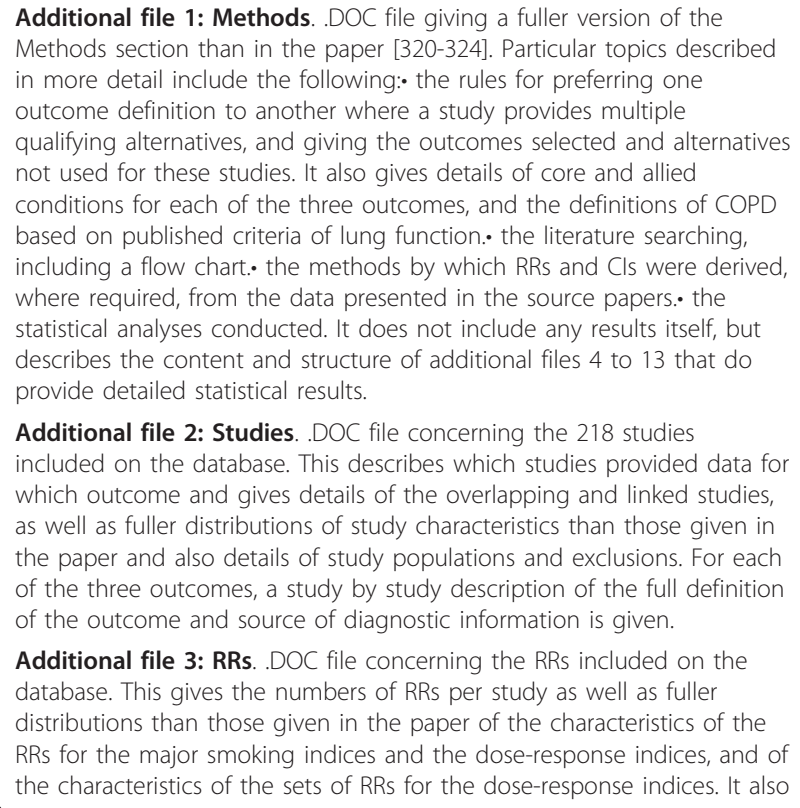

Additional file 2: Studies. DOC file concerning the 218 studies included on the database. This describes which studies provided data for which outcome and gives details of the overlapping and linked studies, as well as fuller distributions of study characteristics than those given in the paper and also details of study populations and exclusions. For each of the three outcomes, a study by study description of the full definition of the outcome and source of diagnostic information is given.

Additional file 3: RRs. .DOC file concerning the RRs included on the database. This gives the numbers of RRs per study as well as fuller distributions than those given in the paper of the characteristics of the RRs for the major smoking indices and the dose-response indices, and of the characteristics of the sets of RRs for the dose-response indices. It also 
lists which studies provide RRs for which indices, and gives details of the results of checking RRs for apparent errors.

Additional file 4: MetaMajorCOPD. RTF file giving the full results of the meta-analyses for the major smoking variables-ever smoking, current smoking, ever or current smoking, and ex smoking-for COPD.

Additional file 5: MetaMajorCB. RTF file giving the full results of the meta-analyses for the major smoking variables for $\mathrm{CB}$.

Additional file 6: MetaMajorEMP. RTF file giving the full results of the meta-analyses for the major smoking variables for EMP.

Additional file 7: MetaDoseCOPD. RTF file giving the full results of the meta-analyses for the dose-related smoking variables-amount smoked, age of starting to smoke, pack-years, duration of smoking, duration of quitting vs. never smoking, and duration of quitting vs. current smokingfor COPD.

Additional file 8: MetaDoseCB. RTF file giving the full results of the meta-analyses for the dose-related smoking variables for CB.

Additional file 9: MetaDoseEMP. RTF file giving the full results of the meta-analyses for the dose-related smoking variables for EMP.

Additional file 10: MetaSumm. XLS file allowing the user readily to view selected results from the full meta-analysis output in additional files 4 to 9 .

Additional file 11: MetaRegressionTables. DOC file giving the full results of the meta-regressions carried out for ever smoking and current smoking.

Additional file 12: DoseSetsList. XLS file listing and plotting the studyspecific sets of dose-response data.

Additional file 13: DoseNotMetaData. .DOC file summarizing the results of the dose-related data that could not be included in the doserelated meta-analyses.

\section{Abbreviations}

ATS: American Thoracic Society; BTS: British Thoracic Society; CB: Chronic Bronchitis; Cl: Confidence Interval; COPD: Chronic Obstructive Lung Disease; ERS: European Respiratory Society; GOLD: Global Initiative for Chronic Obstructive Lung Disease; ICD: International Classification of Diseases; LCL: Lower Confidence Limit; MRC: Medical Research Council; REF: 6 character Reference code used to identify a study; RR: Relative Risk; UCL: Upper Confidence Limit

\section{Acknowledgements}

This research was funded by Philip Morris International, Inc. However the opinions and conclusions of the authors are their own, and do not necessarily reflect the position of Philip Morris International, Inc. We thank John Fry for assistance with the statistical analysis. We also thank Pauline Wassell, Diana Morris and Yvonne Cooper for assistance in typing the various drafts of the paper and obtaining the relevant literature.

\section{Author details}

${ }^{1} \mathrm{P} N$ Lee Statistics and Computing Ltd, Sutton, Surrey, UK. ${ }^{2}$ Independent consultant, Exeter, Devon, UK.

\section{Authors' contributions}

BAF and PNL were responsible for planning the study. Final literature searches were carried out by BAF with PNL's assistance, with some earlier searches conducted by AJT. Data entry was either carried out by AJT and checked by BAF, or carried out by BAF and checked by PNL. Where appropriate, difficulties in interpreting published data or in the appropriate methods for derivation of RRs were discussed by BAF and PNL. The statistical analyses were conducted by BAF along lines discussed and agreed with PNL. PNL drafted the paper, which was then critically reviewed by BAF and AJT.

\section{Competing interests}

PNL, founder of P.N.Lee Statistics and Computing Ltd., is an independent consultant in statistics and an advisor in the fields of epidemiology and toxicology to a number of tobacco, pharmaceutical and chemical companies.This includes Philip Morris International, Inc., the sponsor of this study. BAF is an employee of, and AJT a consultant to, P.N.Lee Statistics and Computing Ltd. All authors read and approved the final manuscript.

Received: 26 November 2010 Accepted: 14 June 2011 Published: 14 June 2011

\section{References}

1. US Surgeon General: The health consequences of smoking. Chronic obstructive lung disease. A report of the Surgeon General Rockville, Maryland: US Department of Health and Human Services; Public Health Service; 1984 [http://www.surgeongeneral.gov/library/reports/index.html], DHHS (PHS) 8450205 .

2. Lee PN, Fry JS: Systematic review of the evidence relating FEV ${ }_{1}$ decline to giving up smoking. BMC Med 2010, 8(84)[http://www.biomedcentral. com/1741-7015/8/84].

3. Pauwels R, Buist A, Calverley P, Kenkins C, Hurd S: Global strategy for the diagnosis, management, and prevention of global initiative for chronic obstructive lung disease. NHLBI/WHO global initiative for chronic obstructive lung disease (GOLD) workshop summary. Am J Respir Crit Care Med 2001, 163:1256-1276.

4. Global Initiative for Chronic Obstructive Disease: Global strategy for the diagnosis, management, and prevention of chronic obstructive pulmonary disease. 2006. Executive summary Medical Communications Resources, Inc; 2006 [http://www.goldcopd.org], (Revised 2006).

5. British Thoracic Society: BTS guidelines for the management of chronic obstructive pulmonary disease. Thorax 1997, 52(Suppl 5):1-28.

6. Siafakas NM, Vermier P, Pride NB, Paoletti P, Gibson J, Howard P Yernault JC, Decramer M, Higenbottam T, Postma DS, et al: Optimal assessment and management of chronic obstructive pulmonary disease (COPD). Eur Respir J 1995, 8:1398-1420.

7. American Thoracic Society: Standardization of spirometry: 1987 update. Am Rev Respir Dis 1987, 136:1285-1298.

8. American Thoracic Society: Lung function testing: selection of reference values and interpretative strategies. Am Rev Respir Dis 1991, 144:1202-1218.

9. American Thoracic Society: Standards for the diagnosis and care of patients with chronic obstructive pulmonary disease. Am J Respir Crit Care Med 1995, 152:S77-5120.

10. Medical Research Council's Committee on the Aetiology of Chronic Bronchitis: Standardized questionaries on respiratory symptoms. Br Med 」 1960, 2(5213):1665.

11. Medical Research Council's Committee on the Aetiology of Chronic Bronchitis: Definition and classification of chronic bronchitis for clinical and epidemiological purposes. Lancet 1965, 1:775-779.

12. Gardner MJ, Altman DG: Statistics with confidence. Confidence intervals and statistical guidelines London: British Medical Journal; 1989.

13. Fleiss $J$ L, Gross AJ: Meta-analysis in epidemiology, with special reference to studies of the association between exposure to environmental tobacco smoke and lung cancer: a critique. J Clin Epidemiol 1991, 44:127-139.

14. Hamling J, Lee P, Weitkunat R, Ambühl M: Facilitating meta-analyses by deriving relative effect and precision estimates for alternative comparisons from a set of estimates presented by exposure level or disease category. Stat Med 2008, 27:954-970.

15. Lee PN: Simple methods for checking for possible errors in reported odds ratios, relative risks and confidence intervals. Stat Med 1999, 18:1973-1981.

16. Egger M, Davey Smith G, Schneider M, Minder C: Bias in meta-analysis detected by a simple, graphical test. BMJ 1997, 315:629-634.

17. Higgins JPT, Thompson SG, Deeks JJ, Altman DG: Measuring inconsistency in meta-analyses. BMJ 2003, 327:557-560.

18. Prescott $E$, Bjerg AM, Andersen PK, Lange P, Vestbo J: Gender difference in smoking effects on lung function and risk of hospitalization for COPD: results from a Danish longitudinal population study. Eur Respir J 1997, 10:822-827.

19. Langhammer A, Johnsen R, Holmen J, Gulsvik A, Bjermer L: Cigarette smoking gives more respiratory symptoms among women than among men. The Nord-Trøndelag Health Study (HUNT). J Epidemiol Community Health 2000, 54:917-922. 
20. Xu X, Wang L: Gender difference in smoking effects on adult pulmonary function. Eur Respir J 1994, 7:477-483.

21. Camilli AE, Burrows B, Knudson RJ, Lyle SK, Lebowitz MD: Longitudinal changes in forced expiratory volume in one second in adults. Effects of smoking and smoking cessation. Am Rev Respir Dis 1987, 135:794-799.

22. Dockery DW, Speizer FE, Ferris BG Jr, Ware JH, Louis TA, Spiro A III: Cumulative and reversible effects of lifetime smoking on simple tests of lung function in adults. Am Rev Respir Dis 1988, 137:286-292.

23. Xu X, Dockery DW, Ware JH, Speizer FE, Ferris BG Jr: Effects of cigarette smoking on rate of loss of pulmonary function in adults: A longitudinal assessment. Am Rev Respir Dis 1992, 146:1345-1348.

24. Forey B, Hamling J, Hamling J, Lee P, editors: International Smoking Statistics. A collection of historical data from 30 economically developed countries. Web edition. Sutton, UK: P N Lee Statistics \& Computing Ltd; 2006 [http://www.pnlee.co.uk/iss.htm].

25. Rodrigues L, Kirkwood BR: Case-control designs in the study of common diseases: updates on the demise of the rare disease assumption and the choice of sampling scheme for controls. Int J Epidemiol 1990, 19:205-213.

26. Alderson MR, Lee PN, Wang R: Risks of lung cancer, chronic bronchitis, ischaemic heart disease, and stroke in relation to type of cigarette smoked. J Epidemiol Community Health 1985, 39:286-293.

27. Alderson MR, Lee PN, Wang R: Risk of lung cancer, chronic bronchitis, ischaemic heart disease, and stroke in relation to type of cigarette smoked, passive smoking and other factors 1986 [http://www.pnlee.co.uk/reflist.htm], [Download ALDERS1986].

28. Alessandri C, Basili S, Violi F, Ferroni P, Gazzaniga PP, Cordova C Hypercoagulability state in patients with chronic obstructive pulmonary disease. Thromb Haemost 1994, 72:343-346.

29. Amigo H, Erazo M, Oyarzun M, Bello S, Peruga A: Tabaquismo y enfermedad pulmonar obstructiva crónica: determinación de fracciones atribuibles (Smoking and chronic obstructive pulmonary disease: attributable risk determination). Rev Med Chil 2006, 134:1275-1282.

30. Anderson DO, Ferris BG, Zickmantel R: The Chilliwack respiratory survey 1963: IV. The effect of tobacco smoking on the prevalence of respiratory disease. CMAJ 1965, 92:1066-1076.

31. Anderson AE, Hernandez JA, Holmes WL, Foraker AG: Pulmonary emphysema: prevalence, severity and anatomical patterns in macrosections, with respect to smoking habits. Arch Environ Health 1966, 12:569-577.

32. Anderson D: Expression of ras (p21) protein in plasma from exposed workers and from patients with lung disease. Int I Hyg Environ Health 2001, 204:55-60.

33. Anderson D, Hughes JA, Cebulska-Wasilewska A, Nizankowska E, Graca B: Ras p21 protein levels in human plasma from patients with chronic obstructive pulmonary disease (COPD) compared with lung cancer patients and healthy controls. Mutat Res 1998, 403:229-235.

34. Auerbach O, Hammond EC, Garfinkel L, Benante C: Relation of smoking and age to emphysema. Whole-lung section study. N Engl J Med 1972, 286:853-857.

35. Auerbach O, Garfinkel L, Hammond EC: Relation of smoking and age to findings in lung parenchyma: a microscopic study. Chest 1974, 65:29-35.

36. Bang KM, Gergen PJ, Carroll M: Prevalence of chronic bronchitis among US Hispanics from the Hispanic Health and Nutrition Examination Survey Examination Survey, 1982-84. Am J Public Health 1990, 80:1495-1497.

37. Beck GJ, Doyle CA, Schachter EN: A longitudinal study of respiratory health in a rural community. Am Rev Respir Dis 1982, 125:375-381.

38. Bednarek M, Plywaczewski R, Jonczak L, Zielinski J: There is no relationship between chronic obstructive pulmonary disease and obstructive sleep apnea syndrome: a population study. Respiration 2005, 72:142-149.

39. Best EWR, Walker CB, Baker PM, Delaquis FM, McGregor JT, McKenzie AC: Summary of a Canadian study of smoking and health. CMAJ 1967, 96:1104-1108.

40. Department of National Health and Welfare Canada: A Canadian study of smoking and health Canada: Department of National Health and Welfare; 1966.

41. Björnsson E, Plaschke P, Norrman E, Janson C, Lundbäck B, Rosenhall A, Lindholm N, Berglund E, Boman G: Symptoms related to asthma and chronic bronchitis in three areas of Sweden. Eur Respir J 1994, 7:2146-2153.
42. Brøgger J, Steen VM, Eiken HG, Gulsvik A, Bakke P: Genetic association between COPD and polymorphisms in TNF, ADRB2 and EPHX1. Eur Respir J 2006, 27:682-688.

43. Brown RG, McKeown T, Whitfield AG: A note on the association between smoking and disease in men in the seventh decade. Br J Prev Soc Med 1957, 11:162-164.

44. Cerveri I, Accordini S, Corsico A, Zoia MC, Carrozzi L, Cazzoletti L, Beccaria $M$, Marinoni A, Viegi $G$, de Marco R: Chronic cough and phlegm in young adults. Eur Respir J 2003, 22:413-417.

45. Chapman RS, Calafiore DC, Hasselblad V: Prevalence of persistent cough and phlegm in young adults in relation to long-term ambient sulfur oxide exposure. Am Rev Respir Dis 1985, 132:261-267.

46. Chen Z-M, Xu Z, Collins R, Li W-X, Peto R: Early health effects of the emerging tobacco epidemic in China. JAMA 1997, 278:1500-1504.

47. Chen $Y$, Breithaupt $K$, Muhajarine N: Occurrence of chronic obstructive pulmonary disease among Canadians and sex-related risk factors. J Clin Epidemiol 2000, 53:755-761.

48. Chen J: Age at diagnosis of smoking-related disease. Health Rep 2003, 14:9-19.

49. Cheng XS, Li JZ, Zhang ZX, Liu GH, Hua Y, Li Q: (The relationship between smoking and the incidence of COPD). Zhonghua Jie He He Hu Xi Za Zhi 1999, 22:290-292.

50. Clément J, van de Woestijne KP: Rapidly decreasing forced expiratory volume in one second or vital capacity and development of chronic airflow obstruction. Am Rev Respir Dis 1982, 125:553-558.

51. Coates EO, Bower GC, Reinstein N: Chronic respiratory disease in postal employees. Epidemiologic survey of a group employed in one building. JAMA 1965, 191:161-166.

52. Cocci F, Miniati M, Monti S, Cavarra E, Gambelli F, Battolla L, Lucattelli M, Lungarella G: Urinary desmosine excretion is inversely correlated with the extent of emphysema in patients with chronic obstructive pulmonary disease. Int J Biochem Cell Biol 2002, 34:594-604.

53. College of General Practitioners: Chronic bronchitis in Great Britain. $\mathrm{Br}$ Med J 1961, 2:973-979.

54. Reid DD, Anderson DO, Ferris BG, Fletcher CM: An anglo-american comparison of the prevelence of bronchitis. Br Med J 1964, 2:1487-1491.

55. Dean G, Lee PN, Todd GF, Wicken AJ: Report on a second retrospective mortality study in North-East England-Part I. Factors related to mortality from lung cancer, bronchitis, heart disease and stroke in Cleveland County, with particular emphasis on the relative risks associated with smoking filter and plain cigarettes London: Tobacco Research Council; 1977, Research Paper 14.

56. Dean G, Lee PN, Todd GF, Wicken AJ: Report on a second retrospective mortality study in North-East England-Part II. Changes in lung cancer and bronchitis mortality and in other relevant factors occurring in areas of NorthEast England, 1963-72 London: Tobacco Research Council; 1978.

57. Dean G, Lee PN, Todd GF, Wicken AJ, Sparks DN: Factors related to respiratory and cardiovascular symptoms in the United Kingdom. J Epidemiol Community Health 1978, 32:86-96.

58. Deane M, Goldsmith JR, Tuma D: Respiratory conditions in outside workers: report on outside plant telephone workers in San Francisco and Los Angeles. Arch Environ Health 1965, 10:323-331.

59. DeJong SR, Veltman RH: The effectiveness of a CNS-led community-based COPD screening and intervention program. Clin Nurse Spec 2004, 18:72-79.

60. de Marco R, Accordini S, Cerveri I, Corsico A, Sunyer J, Neukirch F, Künzli N, Leynaert B, Janson C, Gislason T, et al: An international survey of chronic obstructive pulmonary disease in young adults according to GOLD stages. Thorax 2004, 59:120-125.

61. Cerveri I, Accordini S, Verlato G, Corsico A, Zoia MC, Casali L, Burney P, de Marco R: Variations in the prevalence across countries of chronic bronchitis and smoking habits in young adults. Eur Respir $J$ 2001, 18:85-92.

62. Zock J-P, Sunyer J, Kogevinas M, Kromhout H, Burney P, Antó JM: Occupation, chronic bronchitis, and lung function in young adults. An international study. Am J Respir Crit Care Med 2001, 163:1572-1577.

63. de Torres JP, Campo A, Casanova C, Aguirre-Jaime A, Zulueta J: Gender and chronic obstructive pulmonary disease in high-risk smokers. Respiration 2006, 73:306-310.

64. Dickinson JA, Meaker M, Searle M, Ratcliffe G: Screening older patients for obstructive airways disease in a semi-rural practice. Thorax 1999, 54:501-505. 
65. Doll R, Peto R, Wheatley K, Gray R, Sutherland I: Mortality in relation to smoking: 40 years' observations on male British doctors. BMJ 1994, 309:901-911.

66. Doll R, Hill AB: Mortality in relation to smoking: ten years' observations of British doctors. Br Med J 1964, 1:1399-1410.

67. Doll R, Peto R: Mortality in relation to smoking: 20 years' observations on male British doctors. Br Med J 1976, 2:1525-1536, Erratum Br Med J 1980;971.

68. Doll R, Gray R, Hafner B, Peto R: Mortality in relation to smoking: 22 years' observations on female British doctors. Br Med J 1980, i:967-971.

69. Dontas AS, Jacobs DR, Corcondilas A, Keys A, Hannan P: Longitudinal versus cross-sectional vital capacity changes and affecting factors. $J$ Gerontol 1984, 39:430-438.

70. DoPico GA, Reddan W, Tsiatis A, Peters ME, Rankin J: Epidemiologic study of clinical and physiologic parameters in grain handlers of northern United States. Am Rev Respir Dis 1984, 130:759-765.

71. Ehrlich Rl, White N, Norman R, Laubscher R, Steyn K, Lombard C, Bradshaw D: Predictors of chronic bronchitis in South African adults. Int $J$ Tuberc Lung Dis 2004, 8:369-376.

72. Ekberg-Aronsson M, Pehrsson K, Nilsson JÅ, Nilsson PM, Löfdahl CG: Mortality in GOLD stages of COPD and its dependence on symptoms of chronic bronchitis. Respir Res 2005, 6:98.

73. Enright PL, Kronmal RA, Higgins MW, Schenker MB, Haponik EF: Prevalence and correlates of respiratory symptoms and disease in the elderly. Chest 1994, 106:827-834

74. Enstrom JE, Kabat GC: Environmental tobacco smoke and tobacco related mortality in a prospective study of Californians, 1960-98. BMJ 2003, 326:1057-1061.

75. Ferris BG, Higgins ITT, Higgins MW, Peters JM, Van Ganse WF, Goldman MD: Chronic nonspecific respiratory disease, Berlin, New Hampshire, 19611967: A cross-sectional study. Am Rev Respir Dis 1971, 104:232-244.

76. Anderson DO, Ferris BGJ: Role of tobacco smoking in the causation of chronic respiratory disease. N Engl J Med 1962, 267:787-794.

77. Ferris BJ, Anderson DO: The prevalence of chronic respiratory disease in a New Hampshire town. Am Rev Respir Dis 1962, 864:165-177.

78. Ferris BG, Higgins ITT, Higgins MW, Peters JM: Chronic nonspecific respiratory disease in Berlin, New Hampshire, 1961 to 1967. Am Rev Respir Dis 1973, 107:110-122.

79. Ferris BG Jr, Chen H, Puleo S, Murphy RLH Jr: Chronic nonspecific respiratory disease in Berlin, New Hampshire, 1967 to 1973. A further follow-up study. Am Rev Respir Dis 1976, 113:475-485.

80. Fidan F, Cimrin AH, Ergor G, Sevinc C: Airway disease risk from environmental tobacco smoke among coffeehouse workers in Turkey. Tob Control 2004, 13:161-166

81. Finklea JF, Goldberg J, Hasselblad V, Shy CM, Hayes CG: Prevalence of chronic respiratory disase symptoms in military recruits: Chicago induction center, 1969. Health consequences of sulfur oxides: A report from CHESS, 1970-1971 North Carolina: US Environmental Protection Agency; 1974 [http://www.epa.gov/nscep/], 4-23-4-36.

82. Fletcher CM, Elmes PC, Fairbairn AS, Wood CH: The significance of respiratory symptoms and the diagnosis of chronic bronchitis in a working population. Br Med J 1959, 2:257-266, Erratum Br Med J 1959;2 (5153):708.

83. Forastiere F, Balmes J, Scarinci M, Tager IB: Occupation, asthma, and chronic repiratory symptoms in a community sample of older women. Am J Respir Crit Care Med 1998, 157:1864-1870.

84. Foxman B, Sloss EM, Lohr KN, Brook RH: Chronic bronchitis: prevalence, smoking habits, impact, and antismoking advice. Prev Med 1986, 15:624-631.

85. Fukuchi $Y$, Nishimura M, Ichinose M, Adachi M, Nagai A, Kuriyama T, Takahashi K, Nishimura K, Ishioka S, Aizawa H, et al: COPD in Japan: the Nippon COPD Epidemiology study. Respirology 2004, 9:458-465.

86. Geijer RMM, Sachs APE, Verheij TJM, Salomé PL, Lammers J-WJ, Hoes AW: Incidence and determinants of moderate COPD (GOLD II) in male smokers aged 40-65 years: 5-year follow up. Br J Gen Pract 2006, 56:656-661.

87. Godtfredsen NS, Vestbo J, Osler M, Prescott E: Risk of hospital admission for COPD following smoking cessation and reduction: a Danish population study. Thorax 2002, 57:967-972.

88. Goldberg HE, Finklea JF, Nelson CJ, Steen WB, Chapman RS, Swanson DH, Cohen AA: Prevalence of chronic respiratory disease symptoms in adults:
1970 survey of New York communities. Health consequences of sulfur oxides: A report from CHESS, 1970-1971 North Carolina: US Environmental Protection Agency; 1974 [http://www.epa.gov/nscep/], 5-33-5-47.

89. Gulsvik A: Prevalence and manifestations of obstructive lung disease in the city of Oslo. Scand J Respir Dis 1979, 60:286-296.

90. Gulsvik A: Obstructive lung disease in an urban population. Methodological aspects and prevalence estimates of a cross-sectional survey, and some possible risk factors in Oslo, Norway Norway: University of Oslo; 1979.

91. Gulsvik A: Prevalence of respiratory symptoms in the city of Oslo. Scand J Respir Dis 1979, 60:275-285.

92. Haenszel W, Hougen A: Prevalence of respiratory symptoms in Norway. J Chronic Dis 1972, 25:519-544.

93. Hammond EC: Smoking in relation to the death rates of one million men and women. In Epidemiological approaches to the study of cancer and other chronic diseases. Edited by: Haenszel W. Bethesda, Maryland: U.S. Department of Health, Education, and Welfare. Public Health Service National Cancer Institute; 1966:127-204, National Cancer Institute Monograph 19.

94. Hammond EC: Smoking in relation to mortality and morbidity. Findings in first thirty-four months of follow-up in a prospective study started in 1959. J Natl Cancer Inst 1964, 32:1161-1188.

95. Lee PN, Garfinkel L: Mortality and type of cigarette smoked. J Epidemiol Community Health 1981, 35:16-22.

96. US Surgeon General: Reducing the health consequences of smoking. 25 years of progress. A report of the Surgeon General Rockville, Maryland: US Department of Health and Human Services; Public Health Services; 1989 [http://www.surgeongeneral.gov/library/reports/index.html], DHHS Publication No. (CDC) 89-8411.

97. Thun MJ, Day-Lally C, Myers DG, Calle EE, Flanders WD, Zhu BP Namboodiri MM, Heath CW Jr: Trends in tobacco smoking and mortality from cigarette use in cancer prevention studies I (1959 through 1965) and II (1982 through 1988). In Changes in cigarette-related disease risks and their implications for prevention and control. Edited by: Shopland DR, Burns DM, Garfinkel L, Samet JM. Rockville, Maryland: US Department of Health and Human Services, National Institutes of Health, National Cancer Institute; 1997:305-382[http://cancercontrol.cancer.gov/tcrb/monographs/8/m8_4.pdf], Smoking and Tobacco Control. Monograph No. 8. NIH Pub. No. 97-4213..

98. Hardie JA, Vollmer WM, Buist AS, Bakke P, Morkve O: Respiratory symptoms and obstructive pulmonary disease in a population aged over 70 years. Respir Med 2005, 99:186-195.

99. Harik-Khan Rl, Fleg JL, Wise RA: Body mass index and the risk of COPD. Chest 2002, 121:370-376.

100. Harris-Eze AOC: Smoking habits and chronic bronchitis in Nigerian soldiers. East Afr Med J 1993, 70:763-767.

101. Hawthorne VM, Fry JS: Smoking and health: the association between smoking behaviour, total mortality, and cardiorespiratory disease in west central Scotland. J Epidemiol Community Health 1978, 32:260-266.

102. Hayes CG, Hammer DI, Shy CM, Hasselblad V, Sharp CR, Creason JP, McClain KE: Prevalence of chronic respiratory disease symptoms in adults: 1970 survey of five Rocky Mountain communities. Health consequences of sulfur oxides: A report from CHESS, 1970-1971 North Carolina: US Environmental Protection Agency; 1974 [http://www.epa.gov/nscep/, 319-3-33.

103. Hedman J, Kaprio J, Poussa T, Nieminen MM: Prevalence of asthma, aspirin intolerance, nasal polyposis and chronic obstructive pulmonary disease in a population-based study. Int J Epidemiol 1999, 28:717-722

104. Higgins ITT: Respiratory symptoms, bronchitis, and ventilatory capacity in random sample of an agricultural population. Br Med J 1957, 2:1198-1203.

105. Higgins ITT, Cochran JB: Respiratory symptoms, bronchitis, and disability in a random sample of an agricultural community in Dumfriesshire. Tubercle 1958, 39:296-301.

106. Higgins MW, Keller JB: Trends in COPD morbidity and mortality in Tecumseh, Michigan. Am Rev Respir Dis 1989, 140:S42-S48.

107. Higgins MW, Keller JB, Becker M, Howatt W, Landis JR, Rotman H, Weg JG, Higgins I: An index of risk for obstructive airways disease. Am Rev Respir Dis 1982, 125:144-151.

108. Higgins MW, Keller JB, Metzner HL: Smoking, socioeconomic status and chronic respiratory disease. Am Rev Respir Dis 1977, 116:403-410.

109. Metzner HL, Carman WJ, House J: Health practices, risk factors, and chronic disease in Tecumseh. Prev Med 1983, 12:491-507. 
110. Hirayama T: Smoking and cancer in Japan. A prospective study on cancer epidemiology based on census population in Japan. Results of 13 years follow up. In The UICC Smoking Control Workshop. Edited by: Tominaga S, Aoki K. University of Nagoya Press; 1982:2-8.

111. Hirayama T: Health effects of active and passive smoking. In Smoking and health 1987. Proceedings of the 6th World Conference on Smoking and Health, Tokyo, 9-12 November 1987. Edited by: Aoki M, Hisamichi S, Tominaga S. Amsterdam: Elsevier Science Publishers B.V. (Biomedical Division); 1988:75-86, International Congress Series No. 780.

112. Ho SC, Zhan SY, Tang JL, Chan SG, Woo J: Smoking and mortality in an older Chinese cohort. J Am Geriatr Soc 1999, 47:1445-1450.

113. Lai CKW, Ho SC, Lau J, Yuen YK, Ho SS, Chan CHS, Woo J: Respiratory symptoms in elderly Chinese living in Hong Kong. Eur Respir J 1995, 8:2055-2061.

114. Holland WW, Stone RW: Respiratory disorders in United States east coast telephone men. Am J Epidemiol 1965, 82:95-101.

115. Hollnagel H, Madsen F, Larsen S: Lungesymptomer, lungefunktionstests, rygevaner og erhvervseksponering blandt 40-årige mænd og kvinder i Glopstrup. II. Indbyrdes relationer (Pulmonary symptoms, pulmonary function tests, smoking habits and occupational exposure among 40year old men and women in Glostrup. II. Interrelations). Ugeskr Laeger 1983, 145:118-124.

116. Hollnagel H, Madsen F: Lungesymptomer, lungefunktionstests, rygevaner og erhvervseksponering blandt 40-årige mænd og kvinder i Glostrup. I. Fordelinger og relation til sociale forhold (Pulmonary symptoms, pulmonary function tests, smoking habits and occupational exposure among 40-year old men and women in Glostrup. I. Distribution and relation to sociodemographic variables). Ugeskr Laeger 1983, 145:113-118.

117. House DE, Finklea JF, Shy CM, Calafiore DC, Riggan WB, Southwick JW, Olsen $\sqcup$ : Prevalence of chronic respiratory disease symptoms in adults: 1970 survey of salt lake basin communities. Health consequences of sulfur oxides: A report from CHESS, 1970-1971 North Carolina: US Environmental Protection Agency; 1974 [http://www.epa.gov/nscep/], 2-41-2-54. EPA-650/ 1-74-004.

118. Hozawa A, Billings $J$, Shahar E, Ohira T, Rosamond WD, Folsom AR: Lung function and ischemic stroke incidence: the Atherosclerosis Risk in Communities study. Chest 2006, 130:1642-1649.

119. Shahar E, Folsom AR, Melnick SL, Tockman MS, Comstock GW, Gennaro V, Higgins MW, Sorlie PD, Ko W-J, Szklo M: Dietary n-3 polyunsaturated fatty acids and smoking-related chronic obstructive pulmonary disease. $N$ Engl J Med 1994, 331:228-233.

120. Shahar E, Boland LL, Folsom AR, Tockman MS, McGovern PG, Eckfeldt JH: Docosahexaenoic acid and smoking-related chronic obstructive pulmonary disease. The Atherosclerosis Risk in Communities Study Investigators. Am J Respir Crit Care Med 1999, 159:1780-1785.

121. Hrubec Z, Cederlof R, Friberg L, Horton R, Ozolins G: Respiratory symptoms in twins: effects of residence-associated air pollution, tobacco and alcohol use, and other factors. Arch Environ Health 1973, 27:189-195.

122. Huchon GJ, Vergnenègre $A$, Neukirch F, Brami G, Roche N, Preux P-M: Chronic bronchitis among French adults: high prevalence and underdiagnosis. Eur Respir J 2002, 20:806-812.

123. Huhti E: Prevalence of respiratory symptoms, chronic bronchitis and pulmonary emphysema in a Finnish rural population. Field survey of age group 40-64 in the Harjavalta area. Acta Tuberc Pneumonol Scand 1965, LXI(Suppl 61):1-111.

124. Huhti E, Ikkala J: A 10-year follow-up study of respiratory symptoms and ventilatory function in a middle-aged rural population. Eur $J$ Respir Dis $1980,61: 33-45$

125. Huhti E, Takala J, Nuutinen J, Poukkula A: Chronic respiratory disease in rural men. An epidemiological survey of Hankasalmi, Finland. Ann Clin Res 1978, 10:87-94.

126. Itabashi S, Fukushima T, Aikawa T, Yanai M, Sekizawa K, Sasaki H, Takishima T: Allergic sensitization in elderly patients with chronic obstructive pulmonary disease. Respiration 1990, 57:384-388.

127. Jacobs DR, Adachi H, Mulder I, Kromhout D, Menotti A, Nissinen A, Blackburn H: Cigarette smoking and mortality risk. Twenty-five-year follow-up of the Seven Countries Study. Arch Intern Med 1999, 159:733-740.

128. Jaén Diaz Jl, de Castro Mesa C, Gontán G, Salamanca MJ, López de Castro F: Prevalence of chronic obstructive pulmonary disease and risk factors in smokers and ex-smokers. Arch Bronconeumol 2003, 39:554-558.
129. Jensen EJ, Dahl R, Steffensen F: Bronchial reactivity to cigarette smoke in smokers: repeatability, relationship to methacholine reactivity, smoking and atopy. Eur Respir J 1998, 11:670-676.

130. Jindal SK, Aggarwal AN, Chaudhry K, Chhabra SK, D'Souza GA, Gupta D, Katiyar SK, Kumar R, Shah B, Vijayan VK: A multicentric study on epidemiology of chronic obstructive pulmonary disease and its relationship with tobacco smoking and environmental tobacco smoke exposure. Indian J Chest Dis Allied Sci 2006, 48:23-29.

131. Johannessen A, Omenaas ER, Bakke PS, Gulsvik A: Implications of reversibility testing on prevalence and risk factors for chronic obstructive pulmonary disease: a community study. Thorax 2005, 60:842-847.

132. Bakke PS, Baste V, Hanoa R, Gulsvik A: Prevalence of obstructive lung disease in a general population: relation to occupational title and exposure to some airborne agents. Thorax 1991, 46:863-870.

133. Johannessen A, Omenaas E, Bakke P, Gulsvik A: Incidence of GOLD-defined chronic obstructive pulmonary disease in a general adult population. Int J Tuberc Lung Dis 2005, 9:926-932.

134. Omenaas E, Bakke P, Eide GE, Elsayed S, Gulsvik A: Total serum IgE and $\mathrm{FEV}_{1}$ by respiratory symptoms and obstructive lung disease in adults of a Norwegian community. Clin Exp Allergy 1995, 25:682-689.

135. Joshi RC, Madan RN, Brash AA: Prevalence of chronic bronchitis in an industrial population in North India. Thorax 1975, 30:61-67.

136. Jousilahti P, Vartiainen E, Tuomilehto J, Puska P: Symptoms of chronic bronchitis and the risk of coronary disease. Lancet 1996, 348:567-572.

137. Kachel T: Wpływ naraźenia zawodowego i palenia tytoniu na wyniki badań spirometrycznych oraz objawy przewleklego zapalenia oskrzeli (Effects of occupational exposure and smoking on spirometric tests and symptoms of chronic bronchitis). Pneumonol Alergol Pol 2003, 71:428-439.

138. Kahn HA: The Dorn study of smoking and mortality among U.S. veterans: report on eight and one-half years of observation. In Epidemiological approaches to the study of cancer and other chronic diseases. Edited by: Haenszel W. Bethesda, Maryland: U.S. Department of Health, Education, and Welfare. Public Health Service National Cancer Institute; 1966:1-125, National Cancer Institute Monograph 19.

139. Rogot E, Murray JL: Smoking and causes of death among US Veterans: 16 years of observation. Public Health Rep 1980, 95:213-222.

140. Rogot $E$, Hrubec $Z$ : Trends in mortality from chronic obstructive pulmonary disease among U.S. veterans: 1954 to 1979. Am Rev Respir Dis 1989, 140:S69-S75.

141. Hrubec Z, McLaughlin JK: Former cigarette smoking and mortality among U.S. veterans: a 26-year followup, 1954 to 1980. In Changes in cigaretterelated disease risks and their implications for prevention and control. Edited by: Shopland DR, Burns DM, Garfinkel L, Samet JM. Rockville, Maryland: US Department of Health and Human Services, National Institutes of Health, National Cancer Institute; 1997:501-530[http://cancercontrol.cancer.gov/tcrb/ monographs/8/m8_7.pdf], [Smoking and Tobacco Control. Monograph No. 8] NIH Pub. No. 97-4213.

142. Enstrom JE: Smoking cessation and mortality trends among two United States populations. J Clin Epidemiol 1999, 52:813-825.

143. Dorn HF: The mortality of smokers and nonsmokers. Proceedings of the Social Statistics Section Washington DC: American Statistical Association; 1958, 34-71.

144. Dorn HF: Tobacco consumption and mortality from cancer and other diseases. Public Health Rep 1959, 74:581-593.

145. Karakatsani A, Andreadaki S, Katsouyanni K, Dimitroulis I, Trichopoulos D, Benetou $V$, Trichopoulou A: Air pollution in relation to manifestations of chronic pulmonary disease: a nested case-control study in Athens, Greece. Eur J Epidemiol 2003, 18:45-53.

146. Katancik JA, Kritchevsky S, Weyant RJ, Corby P, Bretz W, Crapo RO, Jensen R, Waterer G, Rubin SM, Newman AB: Periodontitis and airway obstruction. J Periodontol 2005, 76:2161-2167.

147. Kato I, Tominaga S, Suzuki T: Characteristics of past smokers. Int J Epidemiol 1989, 18:345-354.

148. Khoury MJ, Beaty TH, Newill CA, Bryant S, Cohen BH: Geneticenvironmental interactions in chronic airways obstruction. Int J Epidemiol $1986,15: 65-72$

149. Kim DS, Kim YS, Jung K-S, Chang JH, Lim C-M, Lee JH, Uh S-T, Shim JJ, Lew WJ: Prevalence of chronic obstructive pulmonary disease in Korea: a population-based spirometry survey. Am J Respir Crit Care Med 2005, 172:842-847. 
150. Kiraz K, Kart L, Demir R, Oymak S, Gulmez I, Unalacak M, Ozesmi M: Chronic pulmonary disease in rural women exposed to biomass fumes. Clin Invest Med 2003, 26:243-248.

151. Klayton R, Fallat $R$, Cohen $A B$ : Determinants of chronic obstrucitive pulmonary disease in patients with intermediate levels of alpha $1^{-}$ antitrypsin. Am Rev Respir Dis 1975, 112:71-75.

152. Kojima S, Sakakibara H, Motani S, Hirose K, Mizuno F, Ito M, Hashimoto S: Effects of smoking and age on chronic obstructive pulmonary disease in Japan. J Epidemio/ 2005, 15:113-117.

153. Kotaniemi J-T, Latvala J, Lundbäck B, Sovijärvi A, Hassi J, Larsson K: Does living in a cold climate or recreational skiing increase the risk for obstructive respiratory diseases or symptoms? Int J Circumpolar Health 2003, 62:142-157.

154. Kotaniemi J-T, Sovijärvi A, Lundbäck B: Chronic obstructive pulmonary disease in Finland: prevalence and risk factors. COPD 2005, 2:331-339.

155. Krzyzanowski M, Jedrychowski W, Wysocki M: Factors associated with change in ventilatory function and the development of chronic obstructive pulmonary disease in a 13-year follow-up of the Cracow study. Risk of chronic obstructive pulmonary disease. Am Rev Respir Dis 1986, 134:1011-1019.

156. Kubík A: The influence of smoking and other etiopathogenetic factors on the incidence of bronchogenic carcinoma and chronic nonspecific respiratory diseases. Czech Med 1984, 7:25-34.

157. Kuller LH, Ockene JK, Townsend M, Browner W, Meilahn E, Wentworth DN: The epidemiology of pulmonary function and COPD mortality in the multiple risk factor intervention trial. Am Rev Respir Dis 1989, 140(3 Pt 2): S76-S81.

158. Lai CKW, Ran PX, Ko FWS, Liu SM, Zheng JP, Zhong NS, IP MSM: COPD: the chinese experience. Eur Respir J 2006, 27:846-849.

159. Lam TH, He Y, Li LS, He SF, Liang BQ: Mortality attributable to cigarette smoking in China. JAMA 1997, 278:1505-1508.

160. Lam TH, He Y, Shi QL, Huang JY, Zhang F, Wan ZH, Sun CS, Li LS: Smoking, quitting, and mortality in a Chinese cohort of retired men. Ann Epidemiol 2002, 12:316-320.

161. He Y, Taihing L, Shi Q, Huang J, Zhang F, Wan Z, Li L: A prospective study on smoking, quitting and mortality in a cohort of elderly in Xi'an, China. Zhonghua Liu Xing Bing Xue Za Zhi 2002, 23:186-189.

162. Sai X-Y, He Y, Wang B, Meng D-R, Xing Q-J, Xiao D, Wang Y, Yan Y-P: (An 18-year follow up study on the risk factors of deaths regarding chronic obstructive pulmonary disease in Xi'an, China). Zhonghua Liu Xing Bing Xue Za Zhi 2006, 27:765-768.

163. Lambert PM, Reid DD: Smoking, air pollution, and bronchitis in Britain. Lancet 1970, i:853-857.

164. Todd GF, Hunt BM, Lambert PM: Four cardiorespiratory symptoms as predictors of mortality. J Epidemiol Community Health 1978, 32:267-274.

165. Lange P, Nyboe J, Appleyard M, Jensen G, Schnohr P: Relationship of the type of tobacco and inhalation pattern to pulmonary and total mortality. Eur Respir J 1992, 5:1111-1117.

166. Prescott EIB, Lange $P$, Vestbo J: Kronisk ekspektoration og risiko for død ved kronisk obstruktiv lungesygdom (Chronic expectoration and risk of death in chronic obstructive lung disease). Ugeskr Laeger 1996, 158:6456-6460.

167. Lange P, Nyboe J, Appleyard M, Jensen G, Schnohr P: Relation of ventilatory impairment and of chronic mucus hypersecretion to mortality from obstructive lung disease and from all causes. Thorax 1990, 45:579-585.

168. Vestbo J, Lange P: Can GOLD Stage 0 provide information of prognostic value in chronic obstructive pulmonary disease? Am J Respir Crit Care Med 2002, 166:329-332.

169. Løkke A, Lange P, Scharling H, Fabricius P, Vestbo J: Developing COPD: a 25 year follow up study of the general population. Thorax 2006, 61:935-939.

170. Lange P, Parner J, Prescott E, Vestbo J: Chronic bronchitis in an elderly population. Age Ageing 2003, 32:636-642.

171. La Vecchia C, Pagano R, Negri E, Decarli A: Smoking and prevalence of disease in the 1983 Italian National Health survey. Int J Epidemiol 1988, 17:50-55.

172. Lebowitz MD: Smoking habits and changes in smoking habits as they relate to chronic conditions and respiratory symptoms. Am J Epidemiol 1977, 105:534-543.
173. Burrows B, Lebowitz MD: Characteristics of chronic bronchitis in a warm, dry region. Am Rev Respir Dis 1975, 112:365-370.

174. Lebowitz MD, Burrows B: Quantitative relationships between cigarette smoking and chronic productive cough. Int J Epidemiol 1977, 6:107-113.

175. Lebowitz MD: Occupational exposures in relation to symptomatology and lung function in a community population. Environ Res 1977, 14:59-67.

176. Lebowitz MD: The relationship of socio-environmental factors to the prevalence of obstructive lung diseases and other chronic conditions. J Chronic Dis 1977, 30:599-611.

177. Lebowitz MD: Respiratory symptoms and disease related to alcohol consumption. Am Rev Respir Dis 1981, 123:16-19.

178. Lee PN: Mortality from smoking-associated diseases in Great Britain. A statistical analysis of British data from the U.S.A.-U.K.-Norway migrant study Sutton, Surrey: P N Lee Statistics and Computing Ltd; 1979 [http://www. pnlee.co.uk/reflist.htm], [Download LEE1979B]].

179. Liaw K-M, Chen C-J: Mortality attributable to cigarette smoking in Taiwan: a 12-year follow-up study. Tob Control 1998, 7:141-148.

180. Lindberg A, Eriksson B, Larsson L-G, Rönmark E, Sandström T, Lundbäck B: Seven-year cumulative incidence of COPD in an age-stratified general population sample. Chest 2006, 129:879-885.

181. Lindström M, Kotaniemi J, Jönsson E, Lundbäck B: Smoking, respiratory symptoms, and diseases. A comparative study between Northern Sweden and Northern Finland: Report from the FinEsS Study. Chest 2001, 119:852-861.

182. Liu B-Q, Peto R, Chen Z-M, Boreham J, Wu Y-P, Li J-Y, Campbell TC, Chen JS: Emerging tobacco hazards in China: 1. Retrospective proportional mortality study of one million deaths. BMJ 1998, 317:1411-1422.

183. Liu SM, Wang XP, Wang DL, Zhou YM, Lu JC, Zheng JP, Zhong NS, Ran PX: (Epidemiologic analysis of COPD in Guangdong province). Zhonghua Yi Xue Za Zhi 2005, 85:747-752.

184. Wang X, Zhou Y, Zeng X, Liu S, Qiu R, Xie J, Zheng J, Lu J, Zhong N, Ran P: (Study on the prevalence rate of chronic obstructive pulmonary disease in northern part of Guangdong province). Zhonghua Liu Xing Bing Xue Za Zhi 2005, 26:211-213.

185. Lundbäck $B$, Lindberg $A$, Lindström $M$, Rönmark $E$, Jonsson $A C$, Jönsson $E$, Larsson L-G, Andersson S, Sandström T, Larsson K: Not 15 but $50 \%$ of smokers develop COPD?-Report from the Obstructive Lung Disease in Northern Sweden Studies. Respir Med 2003, 97:115-122.

186. Lindberg A, Bjerg-Bäcklund A, Rönmark E, Larsson L-G, Lundbäck B: Prevalence and underdiagnosis of COPD by disease severity and the attributable fraction of smoking. Report from the Obstructive Lung Disease in Northern Sweden Studies. Respir Med 2006, 100:264-272.

187. Lundbäck B, Stjernberg N, Nyström L, Lundbäck K, Lindström M, Rosenhall $L$ : An interview study to estimate prevalence of asthma and chronic bronchitis. Eur J Epidemiol 1993, 9:123-133.

188. Hedlund U, Järvholm B, Lundbäck B: Respiratory symptoms and obstructive lung diseases in iron ore miners: report from the obstructive lung disease in northern Sweden studies. Eur J Epidemiol 2004, 19:953-958.

189. Lundback B, Nystrom L, Rosenhall L, Stjernberg N: Obstructive lung disease in northern Sweden: respiratory symptoms assessed in a postal survey. Eur Respir J 1991, 4:257-266.

190. Mador MJ, Deniz O, Aggarwal A, Kufel TJ: Quadriceps fatigability after single muscle exercise in patients with chronic obstructive pulmonary disease. Am J Respir Crit Care Med 2003, 168:102-108.

191. Magnusson S, Gislason T: Chronic bronchitis in Icelandic males: prevalence, sleep disturbances and quality of life. Scand J Prim Health Care 1999, 17:100-104

192. Manfreda J, Cheang M, Warren CPW: Chronic respiratory disorders related to farming and exposure to grain dust in a rural adult community. Am J Ind Med 1989, 15:7-19.

193. Mannino DM, Gagnon RC, Petty TL, Lydick E: Obstructive lung disease and low lung function in adults in the United States: data from the National Health and Nutrition Examination Survey, 1988-1994. Arch Intern Med 2000, 160:1683-1689.

194. Coultas DB, Mapel D, Gagnon R, Lydick E: The health impact of undiagnosed airflow obstruction in a national sample of United States adults. Am J Respir Crit Care Med 2001, 164:372-377.

195. Mannino DM, Ford ES, Redd SC: Obstructive and restrictive lung disease and functional limitation: data from the Third National Health and Nutrition Examination. J Intern Med 2003, 254:540-547. 
196. Hyman JJ, Reid BC: Cigarette smoking, periodontal disease, and chronic obstructive pulmonary disease. J Periodontol 2004, 75:9-15.

197. Moore BA, Augustson EM, Moser RP, Budney AJ: Respiratory effects of marijuana and tobacco use in a U.S. sample. J Gen Intern Med 2004, 20:33-37.

198. Sisson JH, Stoner JA, Romberger DJ, Spurzem JR, Wyatt TA, Owens-Ream J, Mannino DM: Alcohol intake is associated with altered pulmonary function. Alcohol 2005, 36:19-30.

199. Mannino DM, Buist AS, Petty TL, Enright PL, Redd SC: Lung function and mortality in the United States: data from the First National Health and Nutrition Examination Survey follow up study. Thorax 2003, 58:388-393.

200. Bang KM: Prevalence of chronic obstructive pulmonary disease in blacks. J Natl Med Assoc 1993, 85:51-55.

201. Maranetra KN, Chuaychoo B, Dejsomritrutai W, Chierakul N, Nana A, Lertakyamanee J, Naruman C, Suthamsmai T, Sangkaew S, Sreelum W, et al: The prevalence and incidence of COPD among urban older persons of Bangkok Metropolis. J Med Assoc Thai 2002, 85:1147-1155.

202. Marcus EB, Buist AS, MacLean CJ, Yano K: Twenty-year trends in mortality from chronic obstructive pulmonary disease: the Honolulu heart program. Am J Respir Crit Care Med 1989, 140:S64-S68.

203. Matheson MC, Raven J, Walters EH, Abramson MJ, Ellis JA: Microsomal epoxide hydrolase is not associated with COPD in a community-based sample. Hum Biol 2006, 78:705-717.

204. Mellström D, Rundgren Å, Jagenburg R, Steen B, Svanborg A: Tobacco smoking, aging and health among the elderly: a longitudinal population study of 70-year-old men and an age cohort comparison. Age Ageing $1982,11: 45-58$

205. Menezes AMB, Victora CG, Rigatto M: Chronic bronchitis and the type of cigarette smoked. Int J Epidemiol 1995, 24:95-99.

206. Menezes AMB, Perez-Padilla R, Jardim JRB, Muiño A, Lopez MV, Valdivia G, Montes de Oca M, Talamo C, Hallal PC, Victora CG: Chronic obstructive pulmonary disease in five Latin American cities (the PLATINO study): a prevalence study. Lancet 2005, 366:1875-1881.

207. Menezes AMB, Jardim JR, Pérez-Padilla R, Camelier A, Rosa F, Nascimento O, Hallal PC: Prevalence of chronic obstructive pulmonary disease and associated factors: the PLATINO Study in São Paulo, Brazil. Cad Saude Publica 2005, 21:1565-1573.

208. Meren M, Jannus-Pruljan L, Loit H-M, Põlluste J, Jönsson E, Kiviloog J, Lundbäck B: Asthma, chronic bronchitis and respiratory symptoms among adults in Estonia according to a postal questionnaire. Respir Med 2001, 95:954-964.

209. Miller A, Thornton JC, Anderson HA, Selikoff IJ: Clinical respiratory abnormalities in Michigan. Prevalence by sex and smoking history in a representative sample of the adult population. Chest 1988, 94:1187-1194

210. Milne JS, Williamson J: Respiratory symptoms and smoking habits in older people with age and sex differences. Respiration 1972, 29:359-370.

211. Milne JS, Maule MM, Williamson J: Method of sampling in a study of older people with a comparison of respondents and non-respondents. $\mathrm{Br} J$ Prev Soc Med 1971, 25:37-41.

212. Möller W, Barth W, Kohlhäufl M, Häussinger K, Stahlhofen W, Heyder J: Human alveolar long-term clearance of ferromagnetic iron oxide microparticles in healthy and diseased subjects. Exp Lung Res 2001, 27:547-568.

213. Montnémery P, Ådelroth E, Heuman K, Johannisson A, Johansson S-Å, Lindholm L-H, Lundbäck B, Löfdahl C-G: Prevalence of obstructive lung diseases and respiratory symptoms in southern Sweden. Respir Med 1998, 92:1337-1345.

214. Montnémery P, Bengtsson P, Elliot A, Lindholm L-H, Nyberg P, Löfdahl CG: Prevalence of obstructive lung diseases and respiratory symptoms in relation to living environment and socio-economic group. Respir Med 2001, 95:744-752.

215. Montnémery P, Popovic M, Andersson M, Greiff L, Nyberg P, Löfdahl CG, Svensson C, Persson CGA: Influence of heavy traffic, city dwelling and socio-economic status on nasal symptoms assessed in a postal population survey. Respir Med 2003, 97:970-977.

216. Mueller RE, Keble DL, Plummer J, Walker SH: The prevalence of chronic bronchitic, chronic airway obstruction, and respiratory symptoms in a Colorado city. Am Rev Respir Dis 1971, 103:209-228.

217. Nawa T, Nakagawa T, Kusano S, Nakata H: (Prevalence of emphysematous changes as shown by low-dose spiral CT screening images in 6144 healthy subjects). Nihon Kokyuki Gakkai Zasshi 2002, 40:468-472.
218. Nejjari C, Tessier JF, Letenneur L, Lafont S, Dartigues JF, Salamon R: Determinants of chronic bronchitis prevalence in an elderly sample from south-west of France. Monaldi Arch Chest Dis 1996, 51:373-379.

219. Niepsuj G, Kozielski J, Niepsuj K, Ziora D, Polońska A, Cieślicki J, Rauer R, Dworniczak S, Kami|ski J, Jastrzębski D, et al: Przewlekła obturacyjna choroba płuc u mieszkańców miasta Zabrza (Chronic obstructive pulmonary disease in inhabitants of Zabrze). Wiad Lek 2002, 55(Suppl 1):354-359

220. Nihlén U, Nyberg P, Montnémery P, Löfdahl C-G: Influence of family history and smoking habits on the incidence of self-reported physician's diagnosis of COPD. Respir Med 2004, 98:263-270.

221. Nihlén U, Montnémery P, Lindholm LH, Löfdahl CG: Detection of chronic obstructive pulmonary disease (COPD) in primary health care: role of spirometry and respiratory symptoms. Scand J Prim Health Care 1999, 17:232-237.

222. Nilsson S, Carstensen JM, Pershagen G: Mortality among male and female smokers in Sweden: a 33 year follow up. J Epidemiol Community Health 2001, 55:825-830.

223. Cederlöf R, Friberg L, Hrubec Z, Lorich U: The relationship of smoking and some social covariables to mortality and cancer morbidity. A ten year followup in a probability sample of 55, 000 Swedish subjects age 18-69 Stockholm: Karolinska Institute, Dept of Environmental Hygiene; 1975, Part 1 and Part 2.

224. Carstensen J, Pershagen G, Eklund G: Mortality in relation to cigarette and pipe smoking: 16 years' observation of 25,000 Swedish men. J Epidemiol Community Health 1987, 41:166-172.

225. Ogilvie AG, Newell DJ: Chronic bronchitis in Newcastle-upon-Tyne Edinburgh: E and S Livingstone Limited; 1957.

226. Omori H, Nakashima R, Otsuka N, Mishima Y, Tomiguchi S, Narimatsu A, Nonami Y, Mihara S, Koyama W, Marubayashi T, et al: Emphysema detected by lung cancer screening with low-dose spiral CT: prevalence, and correlation with smoking habits and pulmonary function in Japanese male subjects. Respirology 2006, 11:205-210.

227. Oswald NC, Harold JT, Martin WJ: Clinical pattern of chronic bronchitis. Lancet 1953, 265:639-643

228. Oswald NC, Medvei VC: Chronic bronchitis: the effect of cigarette smoking. Lancet 1955, 2:843-847.

229. Pandey MR: Domestic smoke pollution and chronic bronchitis in a rural community of the Hill Region of Nepal. Thorax 1984, 39:337-339.

230. Pandey MR: Prevalence of chronic bronchitis in a rural community of the Hill Region of Nepal. Thorax 1984, 39:331-336.

231. Peat JK, Woolcock AJ, Cullen K: Decline of lung function and development of chronic airflow limitation: a longitudinal study of nonsmokers and smokers in Busselton, Western Australia. Thorax 1990, 45:32-37.

232. Knuiman MW, James AL, Divitini ML, Ryan G, Bartholomew HC, Musk AW: Lung function, respiratory symptoms, and mortality: results from the Busselton Health Study. Ann Epidemiol 1999, 9:297-306.

233. Pelkonen M, Notkola I-L, Nissinen A, Tukiainen H, Koskela H: Thirty-year cumulative incidence of chronic bronchitis and COPD in relation to 30year pulmonary function and 40-year mortality: a follow-up in middleaged rural men. Chest 2006, 130:1129-1137.

234. Pérez-Padilla R, Regalado J, Vedal S, Pare P, Chapela R, Sansores R, Selman M: Exposure to biomass smoke and chronic airway disease in Mexican women. A case-control study. Am J Respir Crit Care Med 1996, 154(3 Pt 1):701-706.

235. Peto R, Speizer FE, Cochrane AL, Moore F, Fletcher CM, Tinker CM, Higgins ITT, Gray RG, Richards SM, Gilliland J, et al: The relevance in adults of air-flow obstruction, but not of mucus hypersecretion, to mortality from chronic lung disease. Results from 20 years of prospective observation. Am Rev Respir Dis 1983, 128:491-500.

236. Pratt PC, Vollmer TR, Miller JA: Epidemiology of pulmonary lesions in nontextile and cotton textile workers: a retrospective autopsy study. Arch Environ Health 1980, 35:133-138.

237. Price DB, Tinkelman DG, Halbert RJ, Nordyke RJ, Isonaka S, Nonikov D, Juniper EF, Freeman D, Hausen T, Levy ML, et al: Symptom-based questionnaire for identifying COPD in smokers. Respiration 2006, 73:285-295.

238. Tinkelman DG, Price DB, Nordyke RJ, Halbert RJ, Isonaka S, Nonikov D, Juniper EF, Freeman D, Hausen T, Levy ML, et al: Symptom-based questionnaire for differentiating COPD and asthma. Respiration 2006, 73:296-305. 
239. Reid DD, Cornfield J, Markush RE, Seigel D, Pedersen E, Haenszel W: Studies of disease among migrants and native populations in Great Britain, Norway, and the United States. III. Prevalence of cardiorespiratory symptoms among migrants and native-born in the United States. In Epidemiological approaches to the study of cancer and other chronic diseases. Edited by: Haenszel W. Bethesda, Maryland: U.S. Department of Health, Education, and Welfare. Public Health Service National Cancer Institute; 1966:321-346, National Cancer Institute Monograph 19.

240. Renwick DS, Connolly MJ: Prevalence and treatment of chronic airways obstruction in adults over the age of 45. Thorax 1996, 51:164-168.

241. Riccioni G, De Benedictis M, Della VR, Di llio C, Guagnano MT, D'Orazio N: Prevalence and severity of airway obstruction in an Italian adult population. Monaldi Arch Chest Dis 2005, 63:88-92.

242. Rimington J: Cigarette smokers' chronic bronchitis: inhalers and noninhalers compared. Br J Dis Chest 1974, 68:161-165.

243. Rimington J: Phlegm and filters. Br Med J 1972, 2:262-264.

244. Ryder RC, Dunnill MS, Anderson JA: A quantitative study of bronchial mucous gland volume, emphysema and smoking in a necropsy population. J Pathol 1971, 104:59-71.

245. Sargeant $L A$, Jaeckel $A$, Wareham NJ: Interaction of vitamin $C$ with the relation between smoking and obstructive airways disease in EPIC Norfolk. Eur Respir J 2000, 16:397-403.

246. Patel BD, Luben RN, Welch AA, Bingham SA, Khaw K-T, Day NE, Lomas DA, Wareham NJ: Childhood smoking is an independent risk factor for obstructive airways disease in women. Thorax 2004, 59:682-686.

247. Sawicki F: Chronic bronchitis and asthmatic disease in Cracow. In Uses of epidemiology in planning health services. Proceedings of the Sixth International Scientific Meeting of IEA. Savremena Administracija, Belgrade Edited by: Davies AM 1973, 652-662.

248. Sawicki F: Chronic nonspecific respiratory diseases in Cracow. Epidemiological Review 1972, 26:229-250.

249. Schwartz J, Weiss ST: Dietary factors and their relation to respiratory symptoms. The second national health and nutrition examination survey. Am J Epidemiol 1990, 132:67-76.

250. Turkeltaub PC, Gergen PJ: Prevalence of upper and lower respiratory conditions in the US population by social and environmental factors: data from the second National Health and Nutritional Examination Survey, 1976-80 (NHANES II). Ann Allergy 1991, 67:147-154.

251. Shahab L, Jarvis MJ, Britton J, West R: Prevalence, diagnosis and relation to tobacco dependence of chronic obstructive pulmonary disease in a nationally representative population sample. Thorax 2006, 61:1043-1047.

252. Sharp JT, Paul O, Lepper MH, McKean H, Saxton GA Jr: Prevalence of chronic bronchitis in an American male urban industrial population. Am Rev Respir Dis 1965, 91:510-520.

253. Sharp JT, Paul O, McKean H, Best WR: A longitudinal study of bronchitic symptoms and spirometry in a middle-aged, male, industrial population. Am Rev Respir Dis 1973, 108:1066-1077.

254. Shimura S, Andoh Y, Haraguchi M, Shirato K: Continuity of airway goblet cells and intraluminal mucus in the airways of patients with bronchial asthma. Eur Respir J 1996, 9:1395-1401.

255. Shin C, In KH, Shim JJ, Yoo SH, Kang KH, Hong M, Choi K: Prevalence and correlates of airway obstruction in a community-based sample of adults. Chest 2003, 123:1924-1931.

256. Sichletidis L, Tsiotsios I, Gavriilidis A, Chloros D, Kottakis I, Daskalopoulou E, Konstantinidis T: Prevalence of chronic obstructive pulmonary disease and rhinitis in northern Greece. Respiration 2005, 72:270-277.

257. Silva GE, Sherrill DL, Guerra S, Barbee RA: Asthma as a risk factor for COPD in a longitudinal study. Chest 2004, 126:59-65.

258. Lebowitz MD, Postma DS, Burrows B: Adverse effects of eosinophilia and smoking on the natural history of newly diagnosed chronic bronchitis. Chest 1995, 108:55-61.

259. Postma DS, Lebowitz MD: Persistence and new onset of asthma and chronic bronchitis evaluated longitudinally in a community population sample of adults. Arch Intern Med 1995, 155:1393-1399.

260. Dodge $R$, Cline MG, Burrows B: Comparisons of asthma, emphysema, and chronic bronchitis diagnoses in a general population sample. Am Rev Respir Dis 1986, 133:981-986.

261. Sobradillo V, Miravitlles M, Jiménez CA, Gabriel R, Viejo JL, Masa JF, Fernández-Fau L, Villasante C: Estudio IBERPOC en España: prevalencia de síntomas respiratorios habituales y de limitación crónica al flujo aéreo (Epidemiological study of chronic obstructive pulmonary disease in
Spain (IBERPOC): prevalence of chronic respiratory symptoms and airflow limitation). Arch Bronconeumol 1999, 35:159-166.

262. Sobradillo Peña VS, Miravitlles M, Gabriel R, Jiménez-Ruiz CA, Villasante C, Masa JF, Viejo JL, Fernández-Fau L: Geographic variations in prevalence and underdiagnosis of COPD: results of the IBERPOC multicentre epidemiological study. Chest 2000, 118:981-989.

263. Jiménez-Ruiz CA, Sobradillo V, Gabriel R, Viejo JL, Masa JF, Miravitlles M, Villasante C, Fernández-Fau L: Síntomas respiratorios y diagnóstico de EPOC en fumadores de distintas labores de tabaco. Resultados del estudio IBERPOC (Respiratory symptoms and diagnosis of COPD in smokers of various types to tobacco. Results from the IBERPOC study). Arch Bronconeumol 2002, 38:530-535.

264. Jiménez-Ruiz C, Miravitlles M, Sobradillo V, Gabriel R, Viejo JL, Masa JF, Fernández-Fau L, Villasante C: Can cumulative tobacco consumption, FTND score, and carbon monoxide concentration in expired air be predictors of chronic obstructive pulmonary disease? Nicotine Tob Res 2004, 6:649-653.

265. Speizer FE, Fay ME, Dockery DW, Ferris BG Jr: Chronic obstructive pulmonary disease mortality in six US cities. Am J Respir Crit Care Med 1989, 140:S49-S55.

266. Sterling TD, Rosenbaum WL, Weinkam JJ: Risk attribution and tobaccorelated deaths. Am J Epidemiol 1993, 138:128-139.

267. Weinkam JJ, Rosenbaum WL, Sterling TD: Computation of relative risk based on simultaneous surveys: an alternative to cohort and casecontrol studies. Am J Epidemiol 1992, 136:722-729.

268. Stjernberg N, Eklund A, Nyström L, Rosenhall L, Emmelin A, Strömqvist LH: Prevalence of bronchial asthma and chronic bronchitis in a community in northern Sweden; relation to environmental and occupational exposure to sulphur dioxide. Eur J Respir Dis 1985, 67:41-49.

269. Ström K, Janzon L, Mattisson I, Rosberg H-E, Arborelius M: Asthma but not smoking-related airflow limitation is associated with a high fat diet in men: results from the population study "Men born in 1914", Malmö, Sweden. Monaldi Arch Chest Dis 1996, 51:16-21.

270. Suadicani P, Hein HO, Meyer HW, Gyntelberg F: Exposure to cold and draught, alcohol consumption, and the NS-phenotype are associated with chronic bronchitis: an epidemiological investigation of 3387 men aged 53-75 years: the Copenhagen Male Study. Occup Environ Med 2001, 58:160-164.

271. Sutinen S, Vaajalahti P, Pääkkö P: Prevalence, severity, and types of pulmonary emphysema in a population of deaths in a Finnish city. Correlation with age, sex and smoking. Scand J Respir Dis 1978, 59:101-115.

272. Tager I, Tishler PV, Rosner B, Speizer FE, Litt M: Studies of the familial aggregation of chronic bronchitis and obstructive airways disease. Int $J$ Epidemiol 1978, 7:55-62.

273. Tager IB, Speizer FE: Risk estimates for chronic bronchitis in smokers: study of male-female differences. Am Rev Respir Dis 1976, 113:619-625.

274. Tang J-L, Morris JK, Wald NJ, Hole D, Shipley M, Tunstall-Pedoe H: Mortality in relation to tar yield of cigarettes: a prospective study of four cohorts. BMJ 1995, 311:1530-1533.

275. Henley SJ, Thun MJ, Chao A, Calle EE: Association between exclusive pipe smoking and mortality from cancer and other diseases. J Natl Cancer Inst 2004, 96:853-861.

276. Taylor DH, Hasselblad V, Henley SJ, Thun MJ, Sloan FA: Benefits of smoking cessation of longevity. Am J Public Health 2002, 92:990-996.

277. Troisi RJ, Speizer FE, Rosner B, Trichopoulos D, Willett WC: Cigarette smoking and incidence of chronic bronchitis and asthma in women. Chest 1995, 108:1557-1561.

278. Trupin L, Earnest G, San Pedro M, Balmes JR, Eisner MD, Yelin E, Katz PP, Blanc PD: The occupational burden of chronic obstructive pulmonary disease. Eur Respir J 2003, 22:462-469.

279. Eisner MD, Balmes J, Katz PP, Trupin L, Yelin EH, Blanc PD: Lifetime environmental tobacco smoke exposure and the risk of chronic obstructive pulmonary disease. Environ Health 2005, 4:7-14.

280. Tsushima K, Sone S, Yoshikawa S, Furuya S, Yasuo M, Suzuki T, Yamazaki Y, Koizumi T, Fujimoto K, Kubo K: Clinical differences in the Global Initiative for Chronic Obstructive Lung Disease Stage 0. Respir Med 2006, 100:1360-1367.

281. Tverdal A, Thelle D, Stensvold I, Leren P, Bjartveit K: Mortality in relation to smoking history: 13 years' follow-up of 68,000 Norwegian men and women 35-49 years. J Clin Epidemiol 1993, 46:475-487. 
282. Urrutia I, Capelastegui A, Quintana JM, Muñiozguren N, Basagana X, Sunyer J: Smoking habit, respiratory symptoms and lung function in young adults. Eur J Public Health 2005, 15:160-165.

283. Dahl M, Tybjærg-Hansen A, Vestbo J, Lange P, Nordestgaard BG: Elevated plasma fibrinogen associated with reduced pulmonary function and increased risk of chronic obstructive pulmonary disease. Am J Respir Crit Care Med 2001, 164:1008-1011.

284. Viegi G, Paoletti P, Prediletto R, Carrozzi L, Fazzi P, Di Pede F, Pistelli G, Giuntini C, Lebowitz MD: Prevalence of respiratory symptoms in an unpolluted area of Northern Italy. Eur Respir J 1988, 1:311-318.

285. Viegi G, Pedreschi M, Pistelli F, Di Pede F, Baldacci S, Carrozzi L, Giuntini C Prevalence of airways obstruction in a general population: European Respiratory Society vs American Thoracic Society definition. Chest 2000, 117:339S-345S.

286. Vikgren J, Boijsen M, Andelid K, Ekberg-Jansson A, Larsson S, Bake B, Tylén U: High-resolution computed tomography in healthy smokers and never-smokers: a 6-year follow-up study of men born in 1933. Acta Radiol 2004, 45:44-52.

287. Vikgren J, Bake B, Ekberg-Jansson A, Larsson S, Tylén U: Value of air trapping in detection of small airways disease in smokers. Acta Radiol 2003, 44:517-524.

288. Tylén U, Boijsen M, Ekberg-Jansson A, Bake B, Löfdahl CG: Emphysematous lesions and lung function in healthy smokers 60 years of age. Respir Med 2000, 94:38-43.

289. Vineis P, Airoldi L, Veglia F, Olgiati L, Pastorelli R, Autrup H, Dunning A, Garte S, Gormally E, Hainaut P, et al: Environmental tobacco smoke and risk of respiratory cancer and chronic obstructive pulmonary disease in former and never smokers in the EPIC prospective study. BMJ 2005, 330:277-280.

290. Vollmer WM, McCamant LE, Johnson LR, Buist AS: Respiratory symptoms, lung function, and mortality in a screening center cohort. Am J Epidemiol 1989, 129:1157-1169.

291. von Hertzen L, Reunanen A, Impivaara O, Mälkiä E, Aromaa A: Airway obstruction in relation to symptoms in chronic respiratory disease-a nationally representative population study. Respir Med 2000, 94:356-363.

292. Wagena EJ, Kant I, van Amelsvoort LGPM, Wouters EFM, van Schayck CP, Swaen GMH: Risk of depression and anxiety in employees with chronic bronchitis: the modifying effect of cigarette smoking. Psychosom Med 2004, 66:729-734.

293. Kant IJ, Bültmann U, Schröer KAP, Beurskens AJHM, van Amelsvoort LGPM, Swaen GMH: An epidemiological approach to study fatigue in the working population: the Maastricht Cohort Study. Occup Environ Med 2003, 60(Suppl I):i32-i39.

294. Wagena EJ, van Amelsvoort LGPM, Kant I, Wouters EFM: Chronic bronchitis, cigarette smoking, and the subsequent onset of depression and anxiety: results from a prospective population-based cohort study. Psychosom Med 2005, 67:656-660.

295. Wald NJ, Watt HC: Prospective study of effect of switching from cigarettes to pipes or cigars on mortality from three smoking related diseases. BMJ 1997, 314:1860-1863.

296. Wang Q, Takashima S, Wang JC, Zheng LM, Sone S: Prevalence of emphysema in individuals who underwent screening CT for lung cancer in Nagano prefecture of Japan. Respiration 2001, 68:352-356.

297. Watson L, Margetts B, Howarth P, Dorward M, Thompson R, Little P: The association between diet and chronic obstructive pulmonary disease in subjects selected from general practice. Eur Respir J 2002, 20:313-318.

298. Weiss W, Boucot KR, Cooper DA, Carnahan WJ: Smoking and the health of older men. II. Smoking and ventilatory function. Arch Environ Health 1963, 7:538-547.

299. Wen C-P, Tsai SPT, Chen C-J, Cheng T-Y: The mortality risks of smokers in Taiwan: Part I: cause-specific mortality. Prev Med 2004, 39:528-535.

300. Wig KL, Guleria JS, Bhasin RC, Holems E, Vasudeva YL, Singh H: Certain clinical and epidemiological aspects of chronic bronchitis as seen in Northern India. Indian Journal of Chest Diseases 1964, 6:183-194.

301. Wilhelmsen L, Orha I, Tibblin G: Decrease in ventilatory capacity between ages of 50 and 54 in representative samples of Swedish men. Br Med J 1969, 3:553-556

302. Wilson D, Adams R, Appleton S, Ruffin R: Difficulties identifying and targeting COPD and population-attributable risk of smoking for COPD: a population study. Chest 2005, 128:2035-2042.
303. Wilson DH, Chittleborough CR, Kirke K, Grant JF, Ruffin RE: The healthrelated quality of life of male and female heavy smokers. Soz Praventivmed 2004, 49:406-412.

304. Wojtyniak B, Krzyżanowski M, Jędrychowski W: Importance of urban air pollution in chronic respiratory problems. Z Erkr Atmungsorgane 1984, 163:274-284

305. Jedrychowski W: Biological meaning of the prospective epidemiological study on chronic obstructive lung disease and aging. Arch Gerontol Geriatr 1983, 2:237-248.

306. Woods RK, Burton DL, Wharton C, McKenzie GH, Walters EH, Comino EJ, Abramson MJ: Asthma is more prevalent in rural New South Wales than metropolitan Victoria, Australia. Respirology 2000, 5:257-263.

307. Woolf CR: Clinical findings, sputum examinations, and pulmonary function tests related to the smoking habit of 500 women. Chest 1974, 66:652-659.

308. Xiao D, Wang C, Du MJ, Pang BS, Zhang HY, Xiao B, Liu JZ, Weng XZ, Su L, Christiani DC: Relationship between polymorphisms of genes encoding microsomal epoxide hydrolase and glutathione S-transferase P1 and chronic obstructive pulmonary disease. Chin Med J 2004, 117:661-667.

309. Xu F, Yin X, Zhang M, Shen H, Lu L, Xu Y: Prevalence of physiciandiagnosed COPD and its association with smoking among urban and rural residents in regional mainland China. Chest 2005, 128:2818-2823.

310. Yamaguchi S, Kano K, Shimojo N, Sano K, Xu XP, Watanabe H, Kameyama M, Santamaria MJ, Liu SJ, Wang LH, et al: Risk factors in chronic obstructive pulmonary malfunction and "chronic bronchitis" symptoms in Beijing district: a joint study between Japan and China. J Epidemiol Community Health 1988, 43:1-6.

311. Yuan JM, Ross RK, Wang XL, Gao YT, Henderson BE, Yu MC: Morbidity and mortality in relation to cigarette smoking in Shanghai, China: a prospective male cohort study. JAMA 1996, 275:1646-1650.

312. Zieliñski J, Bednarek M: Early detection of COPD in a high-risk population using spirometric screening. Chest 2001, 119:731-736.

313. Górecka D, Bednarek M, Kisło A, Zalewska A, Czechowska U, Jędrzejczak M, Zieliński J: Świadomość nieprawidłowego wyniku spirometrii połączona z poradą antynikotynową zwiększa szansę zaprzestania palenia (Awareness of airflow obstruction together with antismoking advice increases success in cessation smoking). Pneumonol Alergol Pol 2001, 69:617-625.

314. Bednarek M, Pływaczewski R, Górecka D, Puścińska E, Nowiński A, Zieliński J: Wczesne rozpoznawanie POChP badaniem spirometrycznym u palących papierosy mieszkańców Warszawy (Early detection of COPD in smokers from Warsaw using spirometric screening). Pneumonol Alergol Pol 2002, 70:139-147.

315. Zielinski J, Bednarek M, Górecka D: Early detection of COPD by high-risk population screening. Eur Respir J 2006, 27:833-837.

316. Zieliński J, Bednarek M, Górecka D: Naroaowy program wczesnego rozpoznawania i profilaktyki POChP w latach 2000-2002 (National Program of Early Detection and Prevention of COPD in the years 20002002). Pneumonol Alergol Pol 2005, 73:116-121.

317. Bednarek M, Zielínski J, Górecka D: Charakterystyka nałogu palenia wśród uczestników narodowego programu wczesnego rozpoznawania i profilaktyki POChP w latach 2000-2002 (Characteristics of smoking habits in participants of the National Program of Early Detection and Prevention of COPD in the years 2000-2002). Pneumonol Alergol Pol 2005, 73:122-127.

318. Zietkowski Z, Kucharewicz I, Bodzenta-Lukaszyk A: The influence of inhaled corticosteroids on exhaled nitric oxide in stable chronic obstructive pulmonary disease. Respir Med 2005, 99:816-824.

319. Zoia MC, Fanfulla F, Bruschi C, Basso O, de Marco R, Casali L, Cerveri I: Chronic respiratory symptoms, bronchial responsiveness and dietary sodium and potassium: a population-based study. Monaldi Arch Chest Dis 1995, 50:104-108.

320. Altman DG, Machin D, Bryant TN, Gardner MJ: Statistics with confidence. 2 edition. London: BMJ Books; 2000

321. DerSimonian R, Laird N: Meta-analysis in clinical trials. Control Clin Trials 1986, 7:177-188.

322. SAS Institute Inc: The GLM procedure. SAS/STAT user's guide (Online Documentation Version 8) Cary, N.C.; [http://www.sfu.ca/sasdoc/sashtml/stat/ chap30/index.htm]

323. World Health Organisation: In International classification of diseases. Manual of the international statistical classification of diseases, injuries, and causes of 
death. Based on the recommendations of the eighth revision conference, 1965, and adopted by the nineteenth world health assembly. Volume 1. Geneva: WHO; 1967.

324. Office of Population Censuses and Surveys: Mortality statistics: cause. Review of the Registrar General on deaths by cause, sex and age, in England and Wales, 1978 London: HMSO; 1980, Series DH2 no. 5.

\section{Pre-publication history}

The pre-publication history for this paper can be accessed here: http://www.biomedcentral.com/1471-2466/11/36/prepub

doi:10.1186/1471-2466-11-36

Cite this article as: Forey et al: Systematic review with meta-analysis of the epidemiological evidence relating smoking to COPD, chronic bronchitis and emphysema. BMC Pulmonary Medicine 2011 11:36.

Submit your next manuscript to BioMed Central and take full advantage of:

- Convenient online submission

- Thorough peer review

- No space constraints or color figure charges

- Immediate publication on acceptance

- Inclusion in PubMed, CAS, Scopus and Google Scholar

- Research which is freely available for redistribution

Submit your manuscript at www.biomedcentral.com/submit 